\title{
Spatio-temporal dynamics in the anchoring of cilia
}

\author{
Dissertation \\ for the award of the degree \\ "Doctor rerum naturalium"
}

(alternatively: "Doctor of Philosophy" Ph.D. Division of Mathematics and Natural Sciences)

of the Georg-August-Universität Göttingen

within the doctoral program Genes and Development

of the Georg-August University School of Science (GAUSS)

submitted by

Shoba Kapoor

from Kabul - Afghanistan (place of birth)

Göttingen, 31.7.19 


\section{Thesis Committee}

(Name/ Department/ Institution)

Prof. Dr. G. Eichele/ Genes and Behavior/MPI for Biophysical Chemistry

Prof. Dr. S. Hoyer-Fender/ Entwicklungsbiologie/ GZMB

Prof. Dr. E. Bodenschatz/ LFPB/ MPI for Dynamics and self-organization

Members of the Examination Board

(Name/ Department/ Institution)

Reviewer: Prof. Dr. G. Eichele/ Genes and Behavior/ MPI for Biophysical Chemistry

Second Reviewer: Prof. Dr. S. Hoyer-Fender/ Entwicklungsbiologie/ GZMB

Further members of the Examination Board

(Name/ Department/ Institution)

Prof. Dr. E. Bodenschatz/ LFPB/ MPI for Dynamics and self-organization

Prof. Dr. A. Stoykova/ Molecular Developmental Neurobiology/ MPI for Biophysical Chemistry

Prof. Dr. E. A. Wimmer/ Entwicklungsbiologie/ GZMB

Dr. P. Lenart/ Cytoskeletal Dynamics in Oocytes/ MPI Biophysical Chemistry

Date of the oral examination: 20.9.19 


\section{Table of Contents}

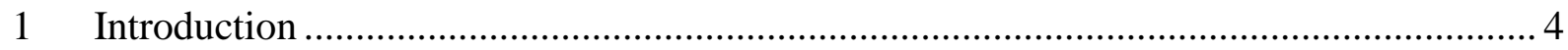

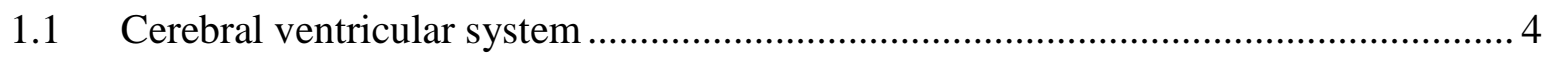

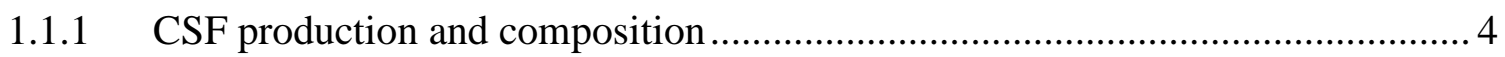

1.1.2 CSF transport and function ...................................................................... 5

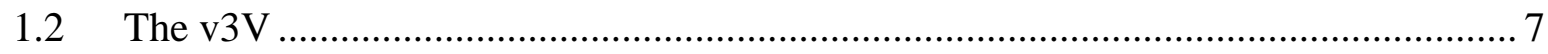

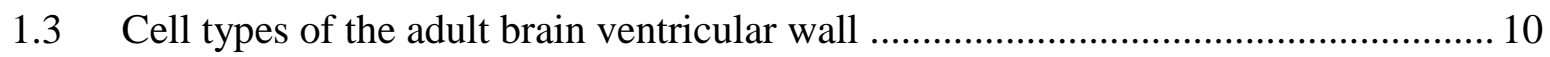

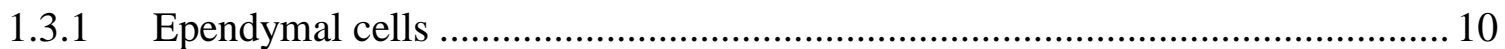

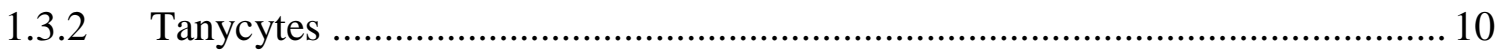

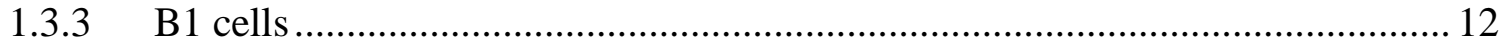

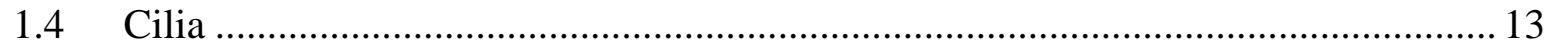

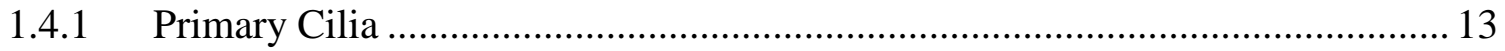

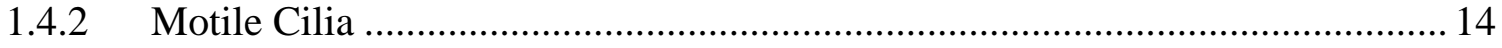

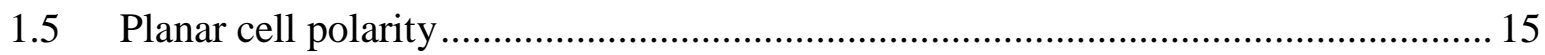

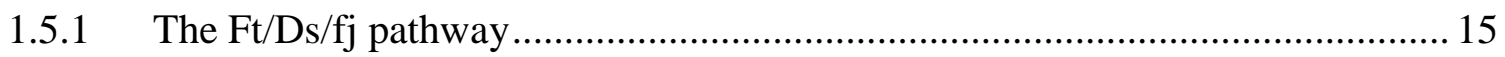

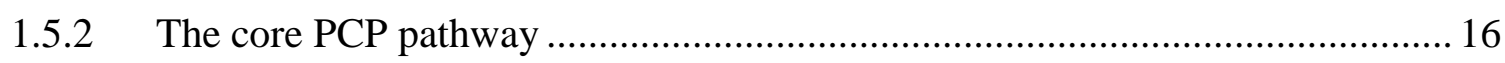

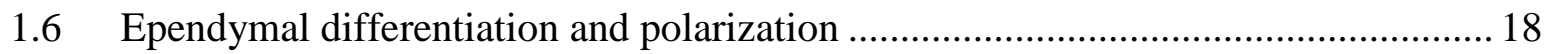

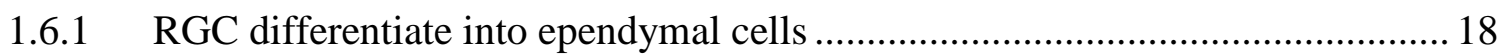

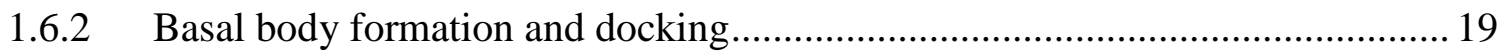

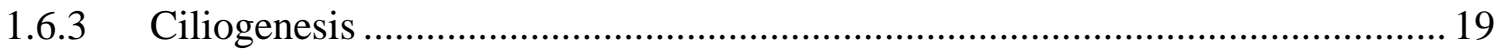

1.6.4 Translational and rotational polarity ........................................................... 19

1.7 Possible changes in flow pattern due to circadian rhythm .................................... 21

1.7.1 Period in mechanisms other than circadian clock ........................................ 22

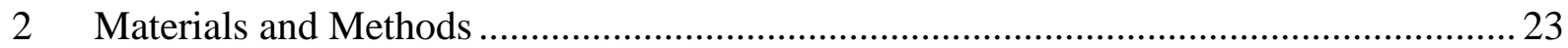

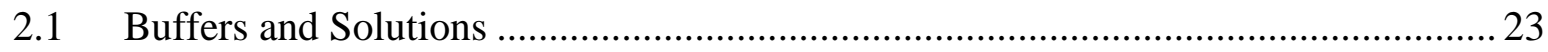

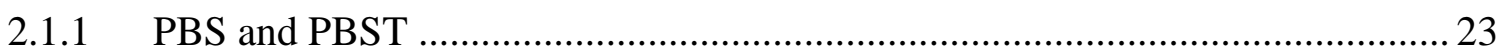

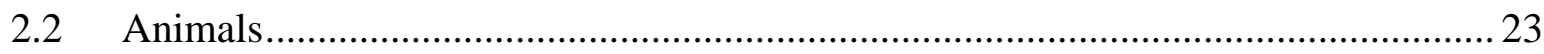




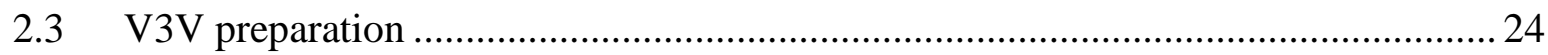

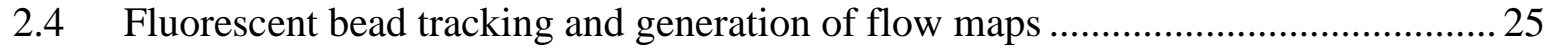

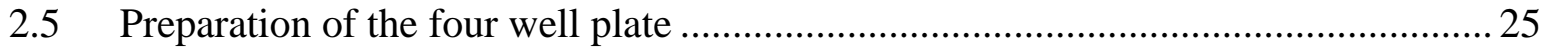

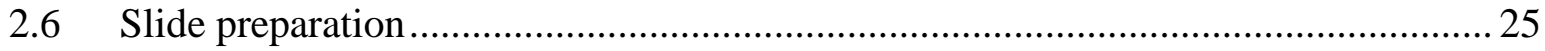

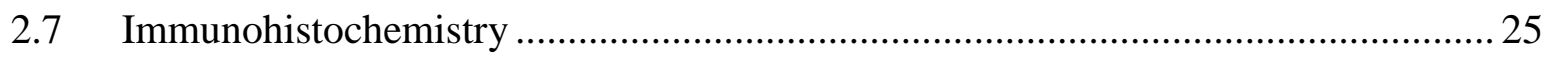

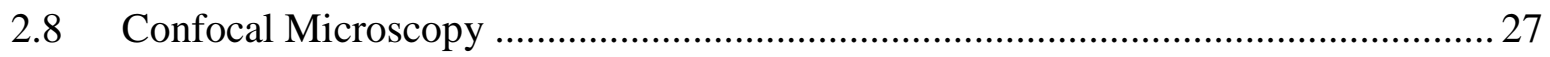

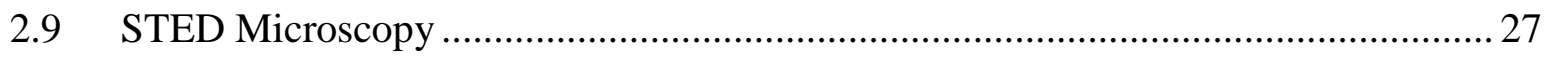

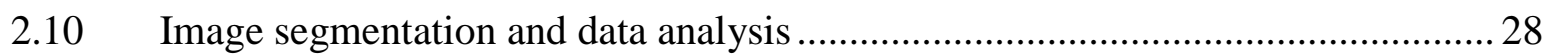

2.10.1 Translational polarity and $\mathrm{v} 3 \mathrm{~V}$ architecture analysis ..................................... 28

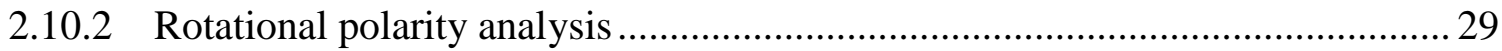

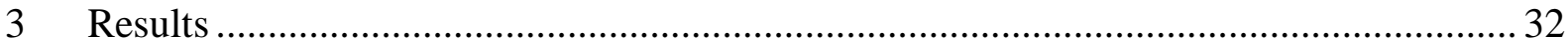

3.1 Planar cell polarity establishes ciliary beating direction ...................................... 32

3.1.1 Translational polarity correlates with the v3V flow-pattern ............................... 32

3.1.2 Vangl 1 expression varies in different $\mathrm{v} 3 \mathrm{~V}$ regions ....................................... 41

3.1.3 The rotational polarity presages the flow-pattern of ependymal cilia................43

3.1.4 The stereotypical pattern of apical area morphology ..................................... 51

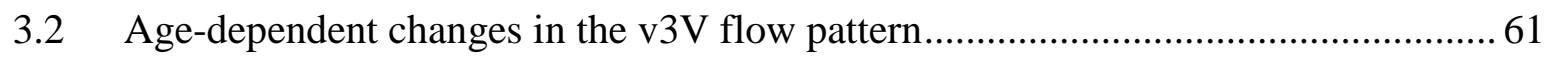

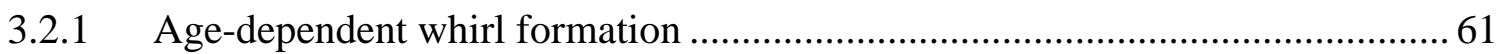

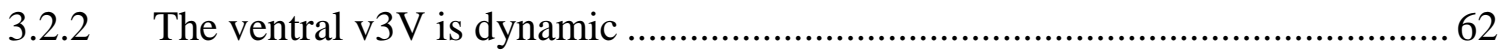

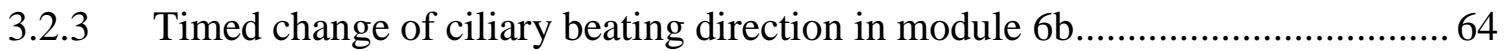

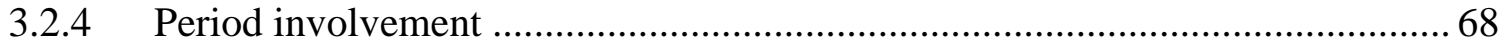

3.2.5 Preliminary results for future investigation.............................................. 71

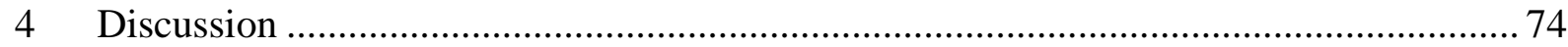

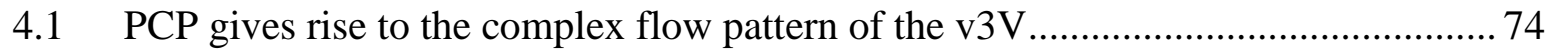

4.1.1 Translational and rotational polarity presage the flow pattern .......................... 74

4.1.2 Vangl1 expression correlates with the flow pattern ....................................... 77

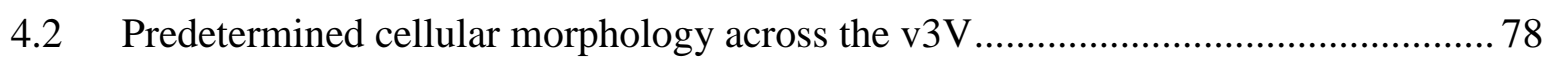

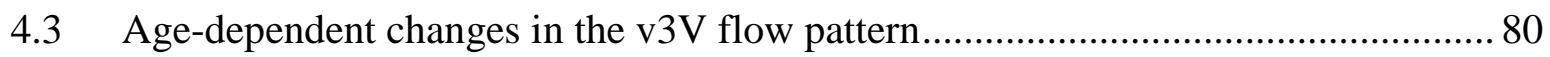


4.4 Period involvement in the timing of postnatal processes

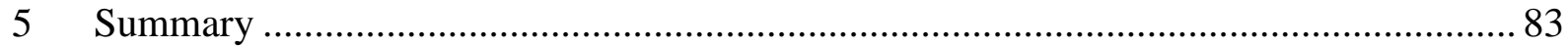

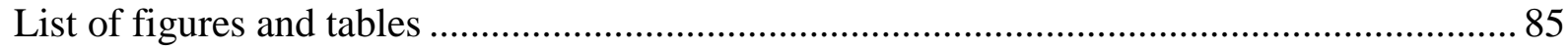

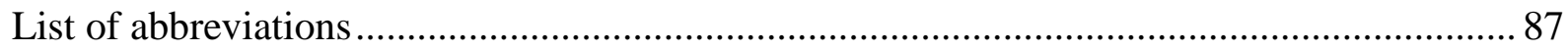

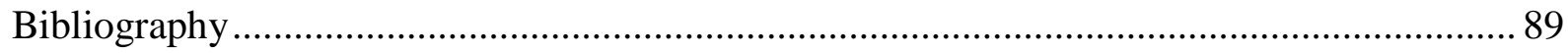

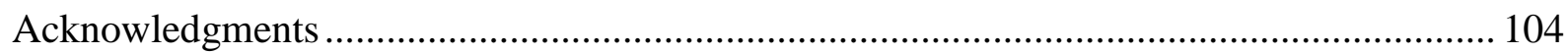

$\mathrm{CV}$ 


\section{Introduction}

\subsection{Cerebral ventricular system}

The ventricular system of the mammalian brain consists of four interconnected cavities, which are filled with cerebrospinal fluid (CSF) that circulates (1). The largest ventricles are the two lateral ventricles $(\mathrm{LV})$ that stretch through the forebrain. Through the foramina of Monro, they are connected with the third ventricle, which is located between the left and right hemispheric thalamus and hypothalamus. The third ventricle is subdivided in a thalamic dorsal (d3V) and a hypothalamic ventral (v3V) area. Caudally the $\mathrm{d} 3 \mathrm{~V}$ is connected to the fourth ventricle through the aqueduct of Sylvius. The fourth ventricle is further connected to the subarachnoid space and the central canal of the spinal cord. Our study focusses on the ventral third ventricle (v3V), located just adjacent to the hypothalami of the left and right hemispheres (Fig.1 A).

\subsubsection{CSF production and composition}

The CSF is mainly produced by the choroid plexus (CP), which, except for the v3V, resides inside each of the cerebral ventricles and is a highly vascularized structure $(2,3)$. In one day, humans produce around $500 \mathrm{ml}$ of CSF and mice around $460 \mu \mathrm{l}(4)$. At any given point, around $100 \mathrm{ml}$ of CSF can be found in humans and $35 \mu \mathrm{l}$ in mice (4).

The cells of the CP epithelium are connected via tight junctions, which establish a diffusion barrier between the blood capillaries in their vicinity and the CSF $(3,5)$. This way, macromolecules of the bloodstream can only enter the CSF via active transport through the CP cells. Interestingly, a recent study showed that the $\mathrm{CP}$ of different ventricles varies in their gene expression pattern. Furthermore, depending on their localization, the different CPs secrete different molecules into the ventricular lumen (6).

However, when the CP was surgically removed in monkeys, it was observed that the CSF volume only decreased to about $40 \%$. Additionally, the tracer sodium ${ }^{24} \mathrm{Na}$ was infused intravenously in those monkeys that lack the CP. In these animals, ${ }^{24} \mathrm{Na}$ was distributed in the CSF in a similar manner as in healthy individuals (7). Later studies pointed towards the importance of microvessels in CSF secretion and reabsorption (8-10). However, whether secreted from the CP or cerebral microvessels, the CSF contains a variety of components including ions, proteins, hormones and extracellular vesicles (10). The concentration of these 
components can vary depending on the position in the brain, the day time or the age of an organism $(6,10-13)$.

\subsubsection{CSF transport and function}

Ependymal cells are cuboidal glial epithelia and are the main cell type that line the walls of the adult brain ventricles and spinal cord canal. These cells possess motile membrane protrusions called cilia on their CSF facing, apical side. In figure 1B we depict how directional movement of these cilia enables the propulsion of CSF (14). While external parameters, such as breathing, heartbeat, and cerebral pressure influence the CSF-flow, near the ventricular walls, it gets transported by the whip-like movement of motile cilia on ependymal cells (14-16). Unlike the CP epithelium, ependymal cells are connected via adherens junctions, which connect the cells loosely and thus allow the transport of CSF between the cells. This way the CSF may exchange fluid with the interstitial space, the area that surrounds neurons and is also filled with fluid (interstitial fluid).

The major pathway describes how the CSF is propelled from the two lateral ventricles through the foramina of Monro into the third ventricle and from there through the aqueduct of Sylvius to the fourth ventricle. Then, the CSF leaves the ventricular system and is transported via the foramina of Magendie and Luschka to the subarachnoid space or to the central canal of the spinal cord (7,9). From the subarachnoid space, the fluid ends up in the lymphatic system or the bloodstream via the perineural space and arachnoid villi, respectively $(8,9,17)$.

The circulating fluid is thought to function as mechanical protection for the central nervous system (CNS) but also as a drainage system for cerebral waste products (10). Whether the CSF flow is involved in directed transport of substances from the CSF into the brain parenchyma remains to be investigated. 


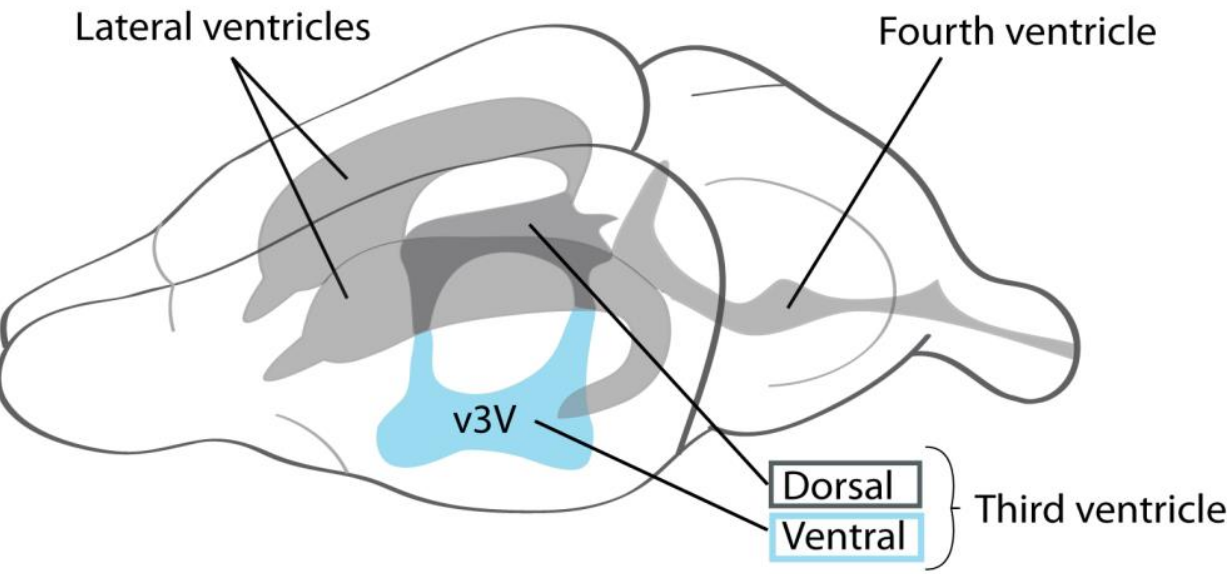

B

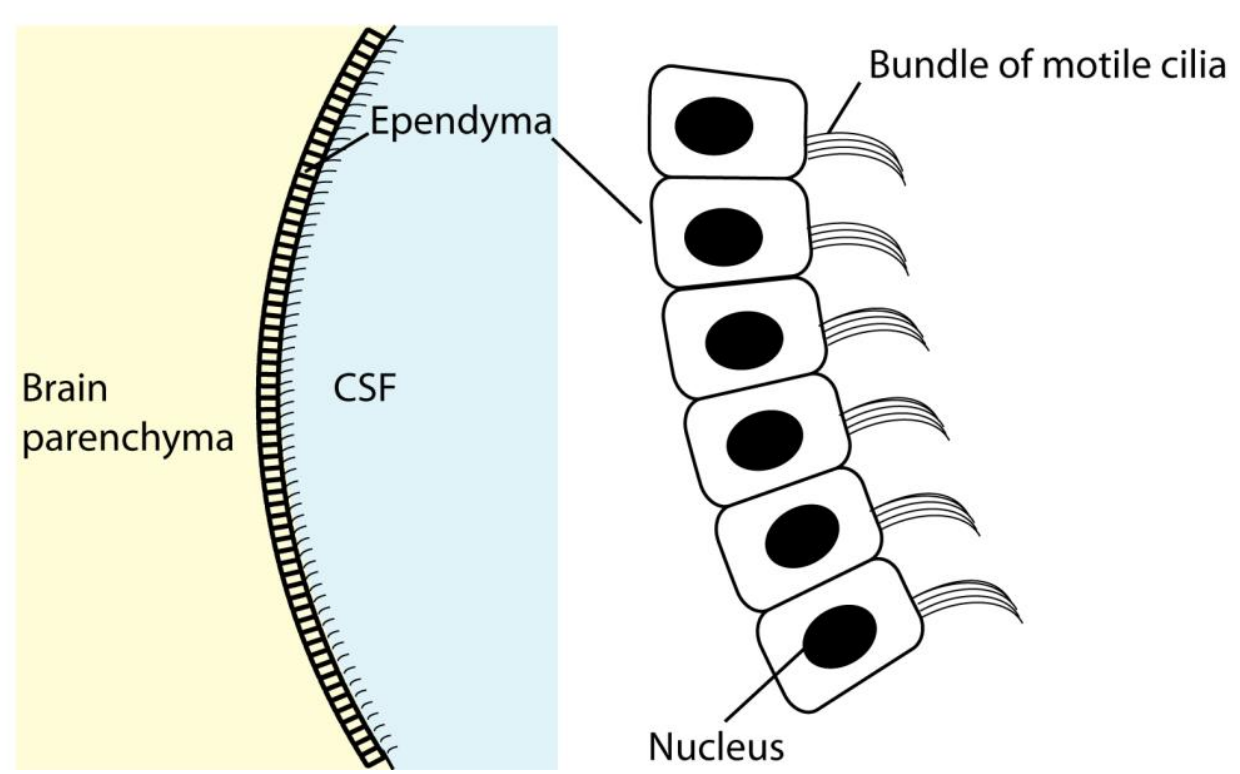

Fig. 1: A) Schematic illustration of the murine cerebral ventricles. The $v 3 \mathrm{~V}$ is highlighted in blue (Modified from www.jax.org). B) Illustration of how ciliated ependymal cells separate the brain parenchyma from the CSF and each possess a bundle of motile cilia. 


\subsection{The v3V}

Near the ventricular walls, the propulsion of CSF can be measured by tracking particle movement that is mediated by directional cilia beating. When this bead tracking analysis was performed to record the near-wall flow pattern of the $\mathrm{v} 3 \mathrm{~V}$ wall, a complex flow was revealed. This flow pattern had eight different flow directions, including straight, bent, opposing, and even whirl-shaped flows (18). In figure 2 we reproduced the findings of this study and use this method of flow observation throughout this thesis. Faubel et al. subdivided the v3V into 8 flow modules. The flow modules 3 and 4 are located near the dorsal center, where their flow pattern opposes one another and forms the so-called separatrix (18). The area, where whirls occur most consistently, is ventral from the separatrix. Studies on the lateral ventricles and tracheal cells show that the direction of cilia beating is regulated by planar cell polarity (PCP) proteins. Planar cell polarity refers to the asymmetric localization of particular proteins that polarize cells of a plane. This polarization also occurs in ependymal cells and establishes the beating direction of the cilia and thus influences the near-wall CSF flow pattern (19).

The basal side of v3V ependymocytes faces the hypothalamus. This anatomical position results from the fact that the cells of the ventricular walls are descendants of radial glial cells (RGC) (20). RGC are progenitors of the neurons in hypothalamic nuclei. This commonality in progenitor makes the flow pattern of the $\mathrm{v} 3 \mathrm{~V}$ particularly interesting. It is possible, that the $\mathrm{v} 3 \mathrm{~V}$ flow pattern could be related to the localization of particular hypothalamic nuclei. Furthermore, the complex flow pattern of the $\mathrm{v} 3 \mathrm{~V}$ may develop to bring solutes to particular areas of the hypothalamus. Examples of this are studies involving intraperitoneal (ip) or intracerebroventricular (i.c.v.) injections of leptin, which regulates appetite and body weight (21). It was found that leptin gets transported from the bloodstream into the CSF via the choroid plexus and from the CSF to particular leptin reactive neurons in the brain parenchyma, such as the neurons of the arcuate nucleus (ARC), the ventromedial hypothalamic nucleus (VMH), and the dorsomedial hypothalamic nucleus (DMH) $(21,22)$. The complexity of flow directions in the $\mathrm{v} 3 \mathrm{~V}$ together with the above-mentioned studies on leptin may be an indication of directional transport of substances to hypothalamic nuclei. In figure 3, we used the Keith B. J. Franklin and George Paxinos brain atlas to reconstruct the areas where the v3V contacts sites of the following hypothalamic nuclei: The median preoptic nucleus (MnPO) and the organum vasculosum of the lamina terminalis (VOLT) are located anterior to the anterior v3V wall. The ventromedial preoptic nucleus/anterior-ventral periventricular nucleus (VMPO/AVPe) and medial preoptic nucleus, med (MPOM) are situated on the basal side of the $\mathrm{v} 3 \mathrm{~V}$ anterior- 
dorsally. Anterior to the $\mathrm{v} 3 \mathrm{~V}$ midline that lies at around Bregma -1, the paraventricular nucleus (PVN) is located dorsally and the Suprachiasmatic nucleus (SCN) ventrally. Next, the ventromedial hypothalamic nucleus dorsomedial the (VMHDM) and the arcuate nucleus (Arc) are positioned adjacent to the ventro-posterior $\mathrm{v} 3 \mathrm{~V}$. The dorsomedial hypothalamic nucleus, diffuse (DMD) and dorsomedial hypothalamic nucleus (DM) locate dorsal to the Arc, adjacent to the dorso-posterior part of the v3V. Finally, the dorsal tuberomammillary nucleus (DTM), and medial mammillary nucleus, med (MM) is positioned next to the most posterior end of the $\mathrm{v} 3 \mathrm{~V}$.

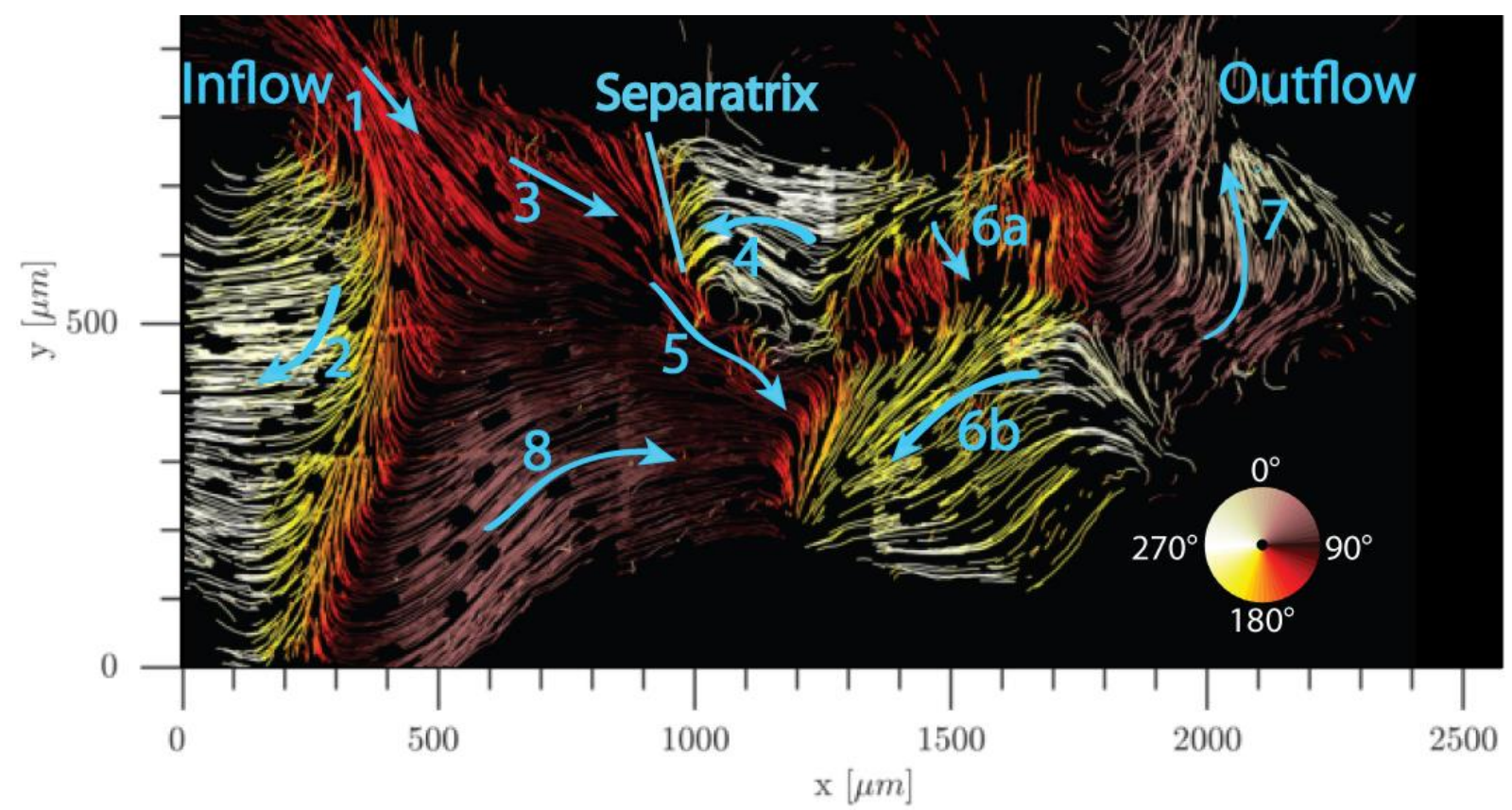

Fig. 2: Flow map of the v3V, in which all modules are marked by blue arrows. The opposing flow directions of module 3 and 4 that form the separatrix. A whirl is observed ventrally from the separatrix. 


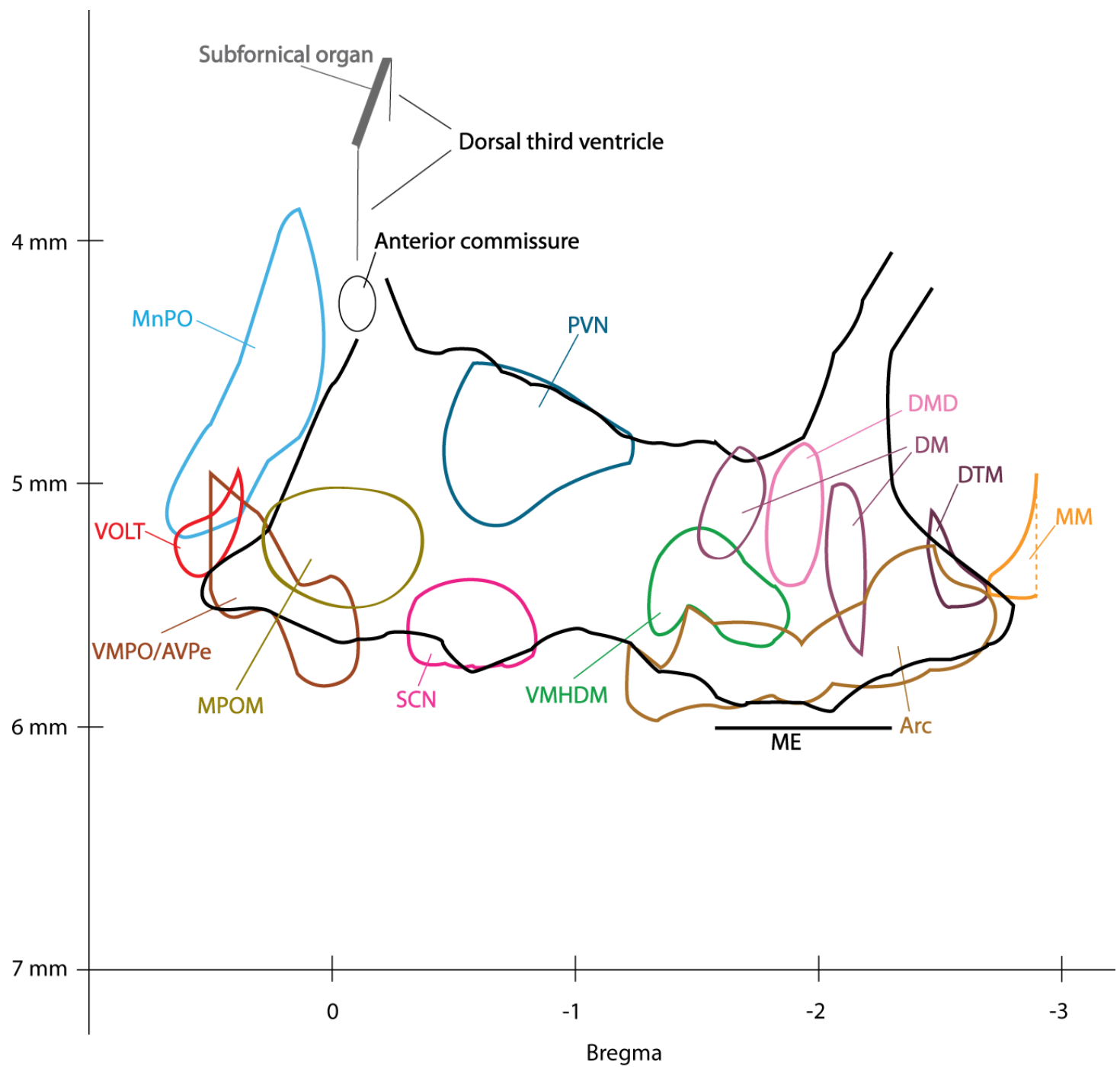

Fig. 3: Schematic illustration of hypothalamic nuclei that are located adjacent to the v3V in the brain parenchyma. This localization was determined by the anatomical structures illustrated in the Keith B. J. Franklin and George Paxinos brain atlas. $\mathrm{MnPO}=$ Median preoptic nucleus; VOLT = Organum vasculosum of the lamina terminalis; $\mathrm{VMPO} / \mathrm{AVPe}=$ Ventromedial preoptic nucleus/Anterio-ventral periventricular nucleus; $\mathrm{MPOM}=$ medial preoptic nucleus, med; PVN = Paraventricular nucleus; SCN $=$ Suprachiasmatic nucleus; VMHDM = Ventromedial hypothalamic nucleus, dorsomedial; Arc $=$ Arcuate nucleus; $\mathrm{DMD}=$ Dorsomedial hypothalamic nucleus, diffuse; $\mathrm{DM}=$ Dorsomedial hypothalamic nucleus; DTM = Dorsal tuberomammillary nucleus; $\mathrm{MM}=$ Medial mammillary nucleus, med 


\subsection{Cell types of the adult brain ventricular wall}

\subsubsection{Ependymal cells}

Ependymocytes were first discovered by Purkinje in 1836 and later described in more detail as cuboidal epithelia (23). Their surface is extended due to microvilli and around $30-60$ motile cilia, which protrude apically to propulse the CSF, as shown in figure 1 and $4(1,23)$.

Ependymal cells are a type of glial cell, which in adults function as a barrier between the CSF and brain-parenchyma and maintain the brain homeostasis by their contribution to the CSF circulation $(24,25)$. The lack of tight junctions between these cells enables the transport of molecules from the CSF to the brain parenchyma or vice versa (10). Furthermore, the expression of secretory molecules like noggin, which promotes neurogenesis, has led to the postulation that ependymal cells may also have secretory functions $(26,27)$.

The ependymal cells are generated from RGC, pluripotent progenitors that during embryogenesis initially produce neurons and later on glial cells (28). Finally, the RGC transform into ependymocytes, expressing the transcriptional factor Foxj1 (20,28). After differentiation, ependymal cells can regain RGC properties upon for example stroke-induced damage or can re-enter cell cycle by the downregulation of Foxj1 $(29,30)$. This indicates that ependymal cells retain some fate-plasticity.

\subsubsection{Tanycytes}

Another cell type in the v3V are the tanycytes that are a specialized RGC. Morphologically they form cuboidal epithelia with only one or two primary cilia facing the CSF. In figure 4 we illustrate how their basal side forms long processes that reach into the brain parenchyma contacting neurons of the hypothalamus (31,32). Depending on their localization and expression profile, tanycytes are classified in 4 subtypes: $\alpha 1, \alpha 2, \beta 1, \beta 2$ (33-35). Each type of tanycyte contacts different nuclei of the hypothalamus (Fig. 5). In the v3V, the processes of the $\alpha 1$ - tanycytes contact neurons of the ventromedial hypothalamic nucleus. The $\alpha 2$ - tanycytes are located ventral from $\alpha 1$ - tanycytes with basal extensions that reach into the ventromedial and the arcuate nucleus. Further ventral are the $\beta 1$ - tanycytes. They contact neurons of the arcuate nucleus and capillaries of the median eminence (ME), which is one of the secretory CVOs involved in the secretion of hypothalamic hormones. The $\beta 2$ - tanycytes are located in the v3V floor and possess processes that also reach to the ME blood vessels (33). Some v3V 
tanycytes (like the ones connected to the ME) are part of the so-called circumventricular organs (CVO), which are either sensory or secretory organs with blood capillaries lacking the blood brain barrier $(31,33)$. The intercellular tight junctions between the tanycytes function as a diffusion barrier. Thus tanycytes are key regulators of blood - hypothalamus and blood - CSF communication $(31,36)$. For example, fasting-induced reduction of the glucose level in the blood is sensed by tanycytes, which in response alters the barrier between the blood and arcuate nucleus (37). Remarkably, recent data indicate that hypothalamic tanycytes are neurogenic progenitors in adult brain neurogenesis. Thus, in addition to the subventricular zone (SVZ) of the lateral ventricles or subgranular zone (SGZ), the tanycytes of the third ventricle define as a new stem cell niche for cerebral injury repair in adults (38).

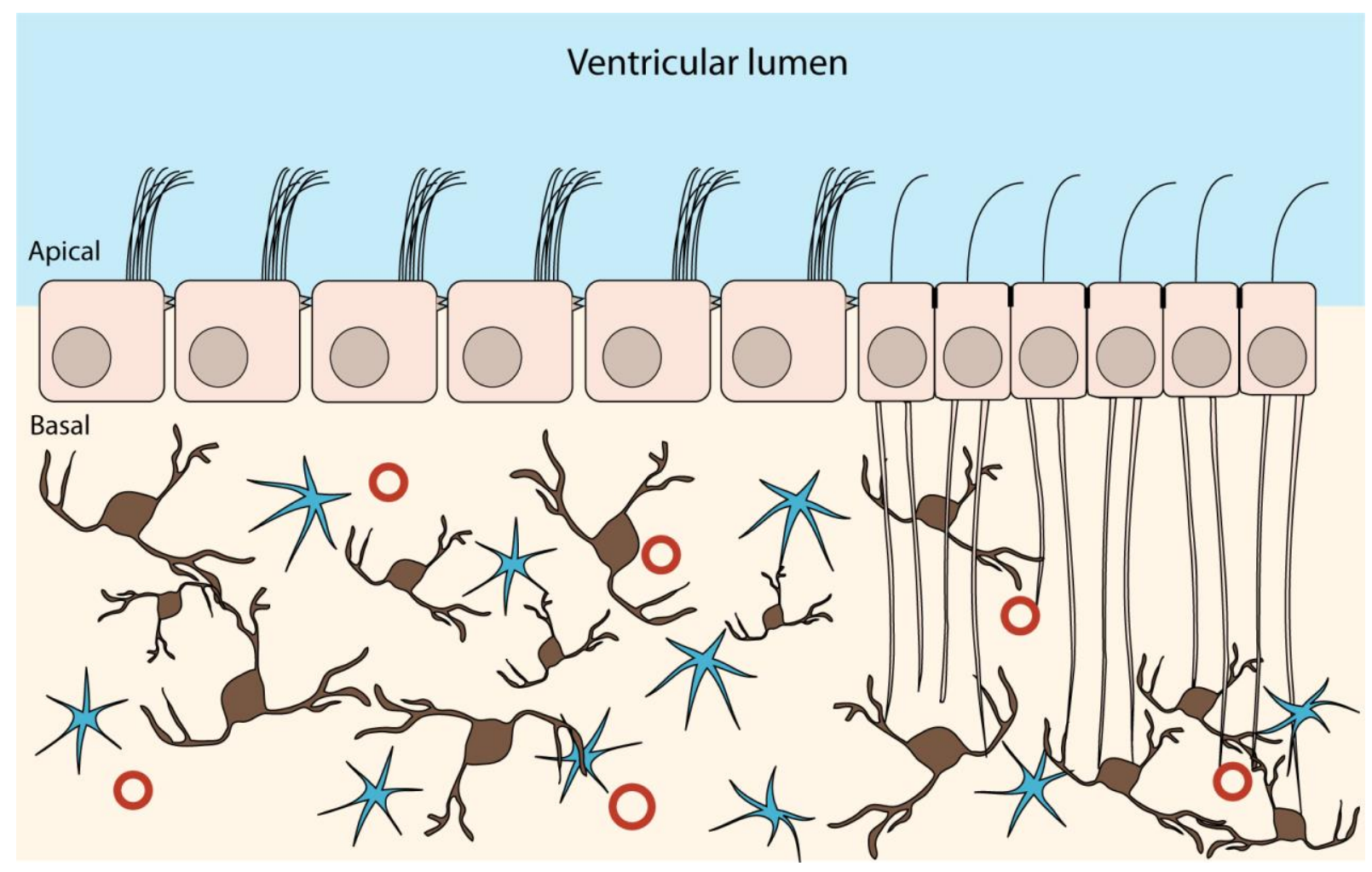

Fig. 4: Schematic illustration of the ventricular wall in the posterior v3V, where ependymal cells meet tanycytes. While ependymal cells are connected loosely via adherence junctions, tanycytes are additionally connected via tight junctions. On the basal side, tanycytes connect to neurons (dark brown) and blood vessels (red). In this region, astrocytes (blue) are also present. 


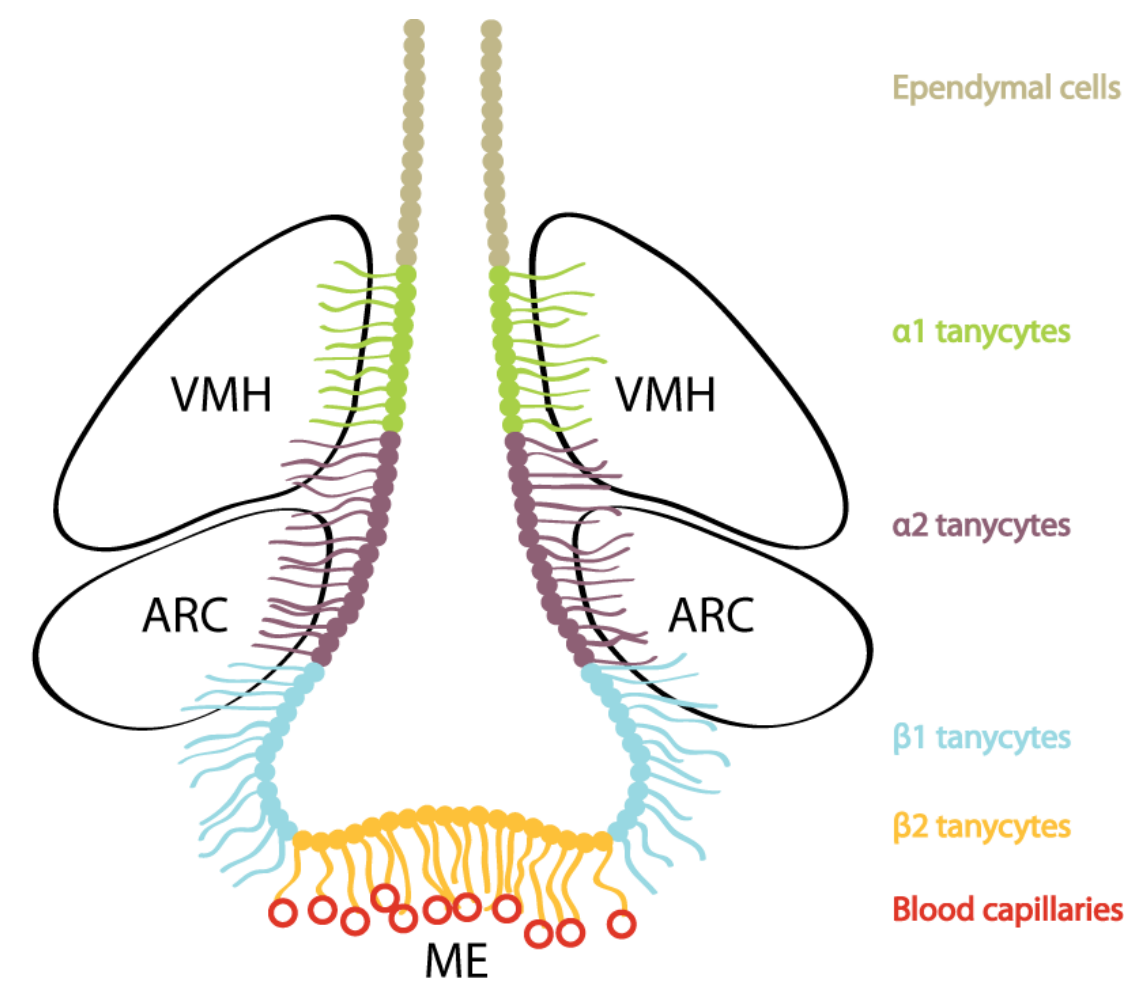

Fig. 5: A simplified scheme of the posterior $\mathrm{v} 3 \mathrm{~V}$, where tanycytes contact hypothalamic nuclei and capillaries of the ME . In this region, $\alpha 1$ tanycytes (green) contact the VMH, $\alpha 2$ tanycytes (violet) contact the VMH and the ARC, $\beta 1$ tanycytes (blue) contact the ARC and capillaries of the ME, while $\beta 2$ tanycytes (orange) only contact the blood capillaries (red) of the ME. Ependymal cells are shown in taupe.

\subsubsection{B1 cells}

As noted above, during development RGC reside in the ventricular zone (VZ) and differentiate into neurons and glial cells, including the ependyma $(20,39,40)$. One of the regions, where adult neurogenesis takes place, is the subventricular zone (SVZ) of the lateral ventricles. In this region, primary neuronal progenitors reside, which give rise to neuroblasts that migrate to the olfactory bulb via the rostral migratory stream (41-45). These cells are termed type B1 cells or astroglia and are surrounded by ependymal cells in a pinwheel-like structure and contact the CSF via a small apical surface and one or two primary cilia $(35,46,47)$. On their basal side, they contact blood vessels via basal processes and have gap junctions with the proliferative astrocytes called B2 cell $(48,49)$. B2 cells are also generated by B1 cells but their function remains elusive (50). Additionally, B1 cells can also give rise to oligodendrocytes (51). The cues B1 cells receive in order to proliferate can originate from cells in their immediate vicinity, the CP, the CSF, the blood stream, and through neurons (46). The SVZ could be a model of how one could picture the tanycyte nice in the $\mathrm{v} 3 \mathrm{~V}$. 


\subsection{Cilia}

Cilia are microtubule based membrane protrusions of cells that have been found across species fulfilling various functions (52). They are docked at a cylindrically shaped basal body on the apical surface of the cell with a microtubule based membrane extension called the axoneme. Figure 6 shows a scheme of axoneme cross sections that enabled the classification of cilia into two types. The axonemes of primary cilia have a $9+0$ structure of microtubule doublets, where 9 pairs of alpha and beta tubulin surround the inner mass of the axoneme. In motile cilia, a 9+2 microtubule structure is observed, which besides the 9 surrounding microtubule doublets, also have an additional central microtubule doublet. The central doublet is thought to interact with radial spokes, which connect the central microtubule doublet with the 9 outer doublets (53-56). Additionally, inner and outer dynein arms are located at the alpha microtubule of the surrounding 9 microtubule doublets. Together with the inner microtubule doublet, the inner and outer dynein arms form the core structures required for ciliary motility $(16,53,57,58)$.

\subsubsection{Primary Cilia}

Primary cilia are slender membrane protrusions and are found on the surface of most mammalian cells $(59,60)$. Anchored at an existing basal body, the axoneme extends from the cell surface and functions as a chemo- or mechanosensory organelle (61). The cilium is attached to the cytoskeleton via a basal body, sub-distal appendages, and a rootlet (62-64). Further apically, cilia possess a transition zone, which functions as a gate for molecules into and out of the axoneme. Inside the axoneme, these molecules are transported via anterograde and retrograde transport, termed intraflagellar transport (IFT). Since protein biosynthesis only occurs in the cytoplasm, the transition zone and IFT play a major role in axon elongation during ciliogenesis and the subsequent homeostasis of ciliary function (65).

The correct composition of proteins in a primary cilium can be essential for the intact function of entire organs. For example, renal cilia possess the mechanosensory transmembrane proteins polycystic kidney disease (Pkd) 1 and Pkd2 to sense fluid flow (66-69). Disruption of pkd1 or pkd2 expression results in the autosomal dominant polycystic kidney disease (ADPKD), resulting in cyst formation and thus enlarged kidneys (70).

Interestingly, pkd1 and pkd2 are also found on primary cilia of the neuronal and glial progenitors RGC. Ohata and collegues propose that RGC sense the external CSF flow prior to 
the differentiation into ependymal cells, which may play a role in determining the beating direction of the motile ependymal cilia (71). This flow that exists before ependymal development may also carry directional cues that polarize ependymal cells.

\subsubsection{Motile Cilia}

Motile cilia are motile membrane protrusions and are structurally the same as flagella in eukaryotes. While flagella can be up to $50 \mu \mathrm{m}$ long and differ in their beating pattern, motile cilia show a whip-like beating, are around $10-15 \mu \mathrm{m}$ long, and occur in bundles on epithelial cells $(53,72,73)$. Cells with multiple motile cilia are termed multiciliated cells (MCC).

Prior to the formation of motile cilia on MCC, basal bodies are synthesized de novo and dock to the apical surface $(59,74,75)$. These basal bodies are connected to one another and the cytoskeleton via a basal foot, the position of which correlates with the beating direction of the cilium (76-78). After docking, the basal bodies are aligned to each other so that the basal foot of each cilium in a bundle points to the same direction. This way, all cilia in a cell beat in one direction and ensure fluid transportation through their whip-like movement $(77,79)$. Additionally, MCC are connected to the cytoskeleton via a rootlet, which is oriented in the opposite direction of the ciliary beat $(76,80)$. Like in primary cilia, the IFT machinery regulates axoneme extension and homeostasis of ciliary protein composition. How exactly the central microtubule doublet is formed and which cell type specific functions cilia fulfill is not understood $(56,58)$.

Depending on the organ, 30 - 300 motile cilia protrude from epithelial cells such as tracheal cells, ciliated fallopian tube cells, or ependymal cells (14,81-83). In the trachea, MCCs transport the mucus produced by secretory cells and clear the respiratory tract from pathogens $(81,84)$. In the fallopian tube, cilia transport the ova to the oviduct, and ependymal cilia ensure CSF circulation $(72,79)$.

Loss of ciliary motility results in numerous diseases, one of which is an autosomal recessive disease called primary ciliary dyskinesia (PCD). Patients suffering from this disease show laterality defects, an impaired respiratory function, infertility, and cognitive disabilities (8588). In the brain, loss of ciliary motility leads to impaired CSF circulation and thus hydrocephalus, resulting in severe cognitive impairments or even death $(16,83,87,89,90)$. 


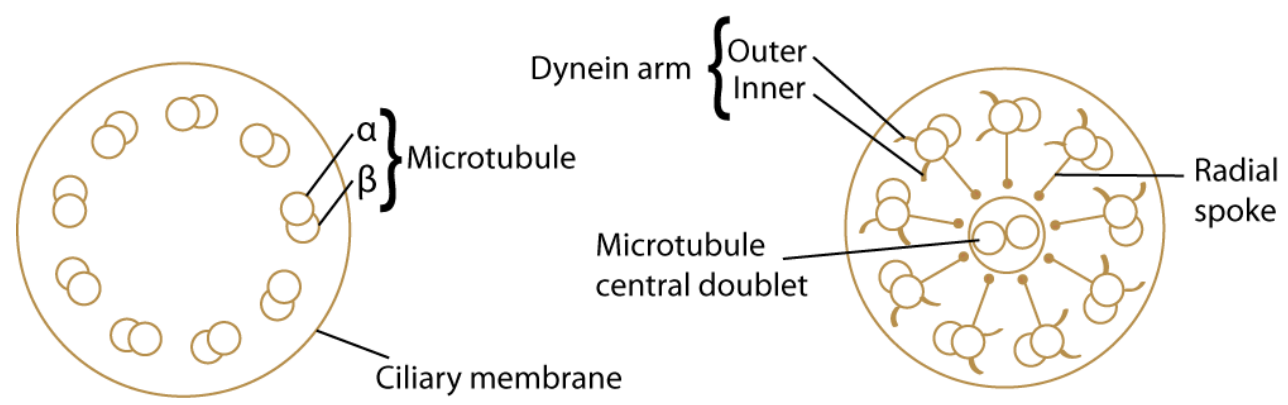

Fig. 6: Schematic illustration of axonemal cross sections of primary (left) and motile (right) cilia. More description. The motile cilium has a more coplex structure, containing a central microtubule doublet, radial spokes and dynein arms.

\subsection{Planar cell polarity}

Planar cell polarity (PCP) is the polarization of cells along an axis in the same direction. PCP is involved in a wide range of processes including directional cell migration and mechanosensation and transduction (91-94). It is extensively studied in Drosophila melanogaster (94-97). Decades of research established that the asymmetric expression of particular transmembrane and cytosolic proteins and their interaction with each other polarizes each cell in a plane $(94,98-100)$. This polarization is a conserved phenomenon in vertebrate and invertebrate tissues involving two main mechanisms: One is the Ft/Ds/fj pathway and the other is the core PCP pathway $(98,101,102)$.

\subsubsection{The Ft/Ds/fj pathway}

The Ft/Ds/fj pathway was termed the global module, in which the atypical cadherins Fat $(\mathrm{Ft})$ and Dachsous (Ds) are located on either side of the cell $(98,102)$. Heterodimers of Ft and Ds form between cells and thus communicate the global polarization from cell to cell. Four-jointed is a golgi ectokinase, which interacts with Ft and Ds and modifies their binding affinities to one another (103). In a plane of cells, $\mathrm{Fj}$ and $\mathrm{Ds}$ are expressed in complementary gradients (104). This tissue wide gradient of expression enables a cellular gradient of Fat $(\mathrm{Ft})$ activity, which together establish tissue wide polarity (98). Ma et al. showed in 2003 that loss of Ft can affect the core PCP pathway in wings of drosophila, which led to the postulation that the Ft/Ds/fj pathway acts upstream of the core pathway (see below). In this view, the global pathway 
establishes a tissue wide polarization, which is then further enhanced by the core PCP pathway acting on a cellular level (105). In contrast, Casal et al. demonstrated that both pathways act independently in the Drosophila abdomen (106). Taking together, the Ft/Ds/fj pathway probably provides a global cue for tissue wide polarization in some tissues, but depending on the cell type it may or may not act upstream of the core PCP pathway.

\subsubsection{The core PCP pathway}

The core PCP pathway consists of transmembrane and cytosolic proteins that interact with each other at the apical side of the cell (Fig. 7). In mammals, this pathway involves the asymmetric localization of Vang gogh like 1-2 (Vangl 1-2), Frizzled 3 and 6 (Fzd 3,6), Cadherin EGF LAG Seven-Pass G-Type Receptor 1-3 (Celsr 1-3), Dishevelled 1-3 (Dvl1-3), Prickle 1-2 (Pk 1-2), and Ankrd6. Vangl 1-2 and Celsr 1-3 are transmembrane proteins that are located on one side of the cells, whereas Fzd 3/6 and Celsr 1-3 are restricted to the opposite side (98,107-110). Ds, $\mathrm{Pk}$ and Ankrd6 are cytosolic proteins, which are also expressed asymmetrically. $\mathrm{Pk} 1-2$ and Ankrd6 are located on the Vangl 1-2 side, whereas 1-3 interact with Fzd 3/6 on the opposite side. Due to monodimers of Celsr 1-3 and heterodimers of the extracellular parts of Vangl and Fzd at cell boundaries, tissue wide polarity is maintained in mammalian epithelia. Within a cell, PCP proteins maintain cellular polarity $(19,108,111)$. 


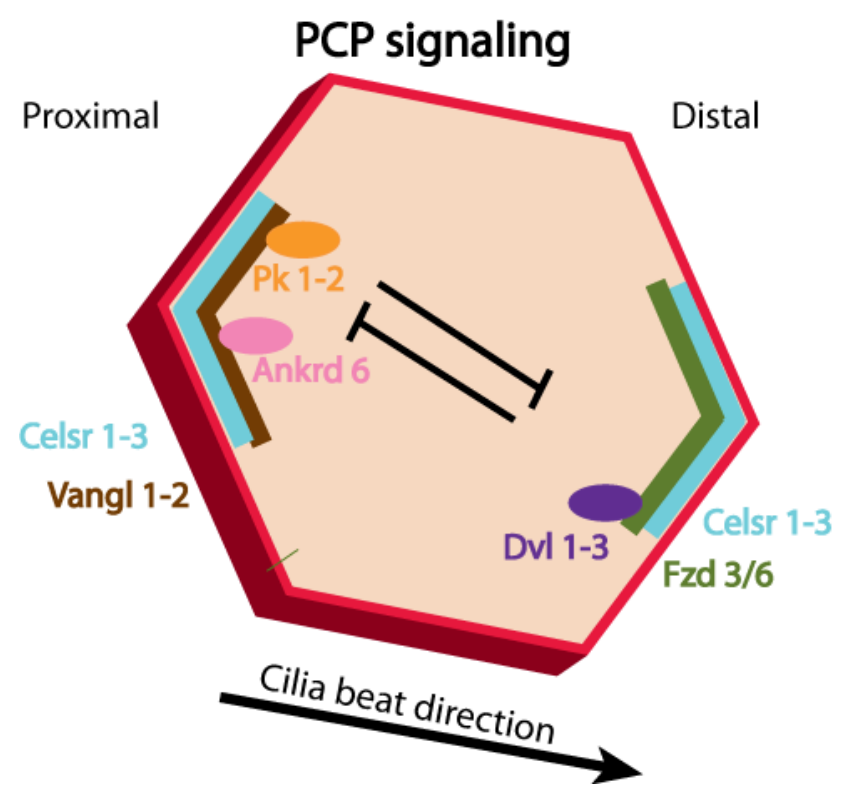

Fig. 7: A simplified scheme of the localization of core PCP proteins. Celsr 1-3 (blue) are transmembrane proteins, present on both sides. Vangl 1-2 (brown) are located on the proximal side and Fzd 3 and 6 (green) on the distal side. Pk1-2 and Ankrd6 are the cytosolic proteins of the proximal side, Dvl 1-3 are located distally. This asymmetric distribution leads to a negative feedback, which propagates PCP in neighboring cells.

\subsubsection{PCP in multiciliated cells}

In multiciliated cells, PCP signaling results in the directional beating of their motile cilia and is the key mechanism in generating a unidirectional flow $(19,110,112,113)$. Figure 8 shows how in these cells, each cilium is polarized and possesses a basal foot at its basal body. The beating direction of each cilium correlates with the position of the basal foot and was termed rotational polarity $(108,114-117)$. Whether in the Xenopus epidermis, mammalian trachea or ependymal cells, all cilia of a cell and all cells of a plane are oriented in the same direction, showing tissue wide polarity $(19,118,119)$. In addition to the rotational and tissue wide polarity, ependymal cells also show an additional polarization, in which bundles of ependymal cilia are localized on one side of the apical surface. This polarity was termed translational polarity (Fig. 8), the direction of which is determined by drawing a vector from the cell centroid to the centroid of the cilia bundle (120). 

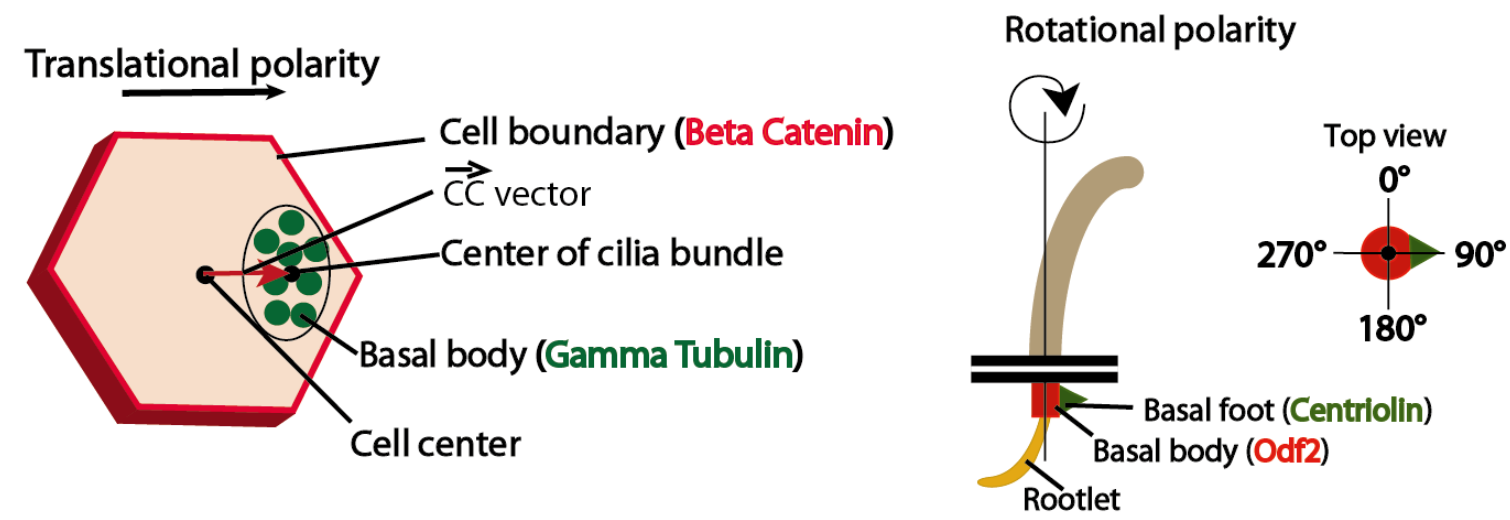

Fig. 8: Schematic illustration of the translational (left) and rotational polarity (right) in ependymal cells. A red vector ( $\overrightarrow{\boldsymbol{C C}}$ vector) drawn from the cell centroid to the centroid of the cilia bundle defines the translational polarity direction of a cell. The rotational polarity direction of a cilium is defined by the basal foot position. The top view illustrates that the rotational polarity of the depicted cilium is in the direction of $90^{\circ}$.

\subsection{Ependymal differentiation and polarization}

\subsubsection{RGC differentiate into ependymal cells}

In figure 9 we illustrate the process of ependymal differentiation and polarization in the lateral ventricles. RGC are progenitors that first give rise to neurons that migrate into the cortical plate but at later stages of development differentiate into ependymal cells (121). In the lateral ventricles, this differentiation occurs between embryonic day 12 (E12) and postnatal day (P) 21 (20). The RGC possess one primary cilium, which is initially located in the center of their apical surface. When these cells become postmitotic, their cilium relocates to one side of the cell and thus establishes the translational polarity (120). It is yet unclear, whether the relocation of the cilium itself could signalize core PCP proteins to be expressed asymmetrically (27). Following the known differentiation gradient in the brain, in the lateral ventricles, the most caudally located RGC differentiate into pre-ependymal cells at around E12 and by P4 the ventricular whole wall is decorated with pre-ependymal cells (20). In this time, these cells express both the radial glial marker GLAST and the ependymal marker S100 $\beta$ and CD24 (20). 


\subsubsection{Basal body formation and docking}

In the course of maturation, BBs are formed de novo by deuterosomes/procentrioles, which are proposed to be derived from the nuclear membrane of the pre-ependymal cells $(20,59)$. A recent finding shows that even though RGC differentiate into ependymal cells and do not undergo mitosis, the mitotic oscillators mediate BB formation. Al Jord et al. divide this procedure into 3 phases. In the amplification phase (A phase) procentrioles are formed, during the growth phase (G phase) these centrioles grow, and during the disengagement phase ( $D$ phase), they detach from the nuclear membrane $(122,123)$. Mediated by the activation of the hexameric protein non-muscle myosin II (NMII), the BB then migrate and dock to the apical surface (124). Another factor involved in the docking is the transcriptional factor forkhead box protein J1 (Foxj1). Gomperts et al. ablated Foxj1 in pulmonary cells, which lead to defects in basal body docking. Interestingly, these mutants also showed a defect in ciliogenesis (125). The link between basal body docking and PCP was, among others, provided by Park and colleagues where they show that Dvl2 is located at basal bodies, which together with the PCP effector protein inturned activates the rho GTPase. This mediates the interaction of the basal bodies and membrane bound vesicles and thus the apical membrane docking (126,127). Although these studies were performed on MCC of the pulmonary system, these mechanisms probably also describe the basal body docking in ependymal cells.

\subsubsection{Ciliogenesis}

As mentioned above, the process of ciliogenesis involves the activation of Foxj1, which is also known as $\mathrm{H} 4 \mathrm{H}-4$ and is expressed in ciliated tissues including ependymal cells $(125,128-131)$. Avasthi and Marshall discussed in 2012, how hundreds of proteins are expressed and accumulate at the ciliary base to initiate ciliogenesis (132). Via intraflagellar transport, these proteins then selectively enter into the growing axoneme (65). At this point, the basal foot of each cilium in an emerging bundle points towards a different direction, which means that the newly formed cilia do not yet beat in an aligned manner.

\subsubsection{Translational and rotational polarity}

Once the cilia are formed, they translocate to that side of the cell, where the transmembrane PCP protein Fzd is located and where initially the RGC primary cilium was displaced $(19,120)$. 
Furthermore, all cilia also align to one another in order to achieve the same beating direction (rotational polarity). These polarities are mediated by the interplay of actin and microtubule dynamics with the core PCP proteins (76,108,120,124,133-135).

Tyrosinated microtubules (tyr - MT) are polarized in developing tracheal MCC, where their plus end is enriched at the distal side of the cell and regulates the asymmetric localization of core PCP proteins (109). In Xenopus MCC it was demonstrated, how polarized cells refine actin and microtubule lattices, which connect each cilium to one another and are key factors for the establishment of rotational polarity $(76,80)$.

In ependymal cells, the relationship between PCP and cytoskeletal elements was recently demonstrated in a study, where the authors show that perturbation of a Dvl associated protein called Daple affects the translational and rotational polarization (133). In developing ependymal cells, polarized tyr - MT are found in wild-type individuals but are absent when Daple is perturbed. Loss of microtubule dynamics affects the ependymal polarity in two ways: First, it disrupts the displacement of the primary cilium in the post-mitotic RGC, which is thought to lead to a misplacement of the later developing motile cilia. Second, core PCP proteins Fzd 6 and Dvl 2 are disarranged, which is suggested to cause the loss of rotational polarity (133). Dvl interaction with microtubules and Fzd is a key factor in the establishment of rotational polarity. For example, in tracheal MCC, Dvl 2 was found to be located at the ciliary base and Dvl 1 and 3 on the distal side of the cell interacting with Fzd 3 and $6(109,126)$. In addition, time-lapse imaging of developing ependymal cells in Dvl 2 morphants showed defects in the rotational polarity. Furthermore, triple knockout of Dvl 1-3, was demonstrated to perturb the rotational polarity in ependymal cells (108).

Another interesting study on the lateral ventricles in PCP mutants showed that the molecular machinery of the cilia bundle displacement is not affected when the transmembrane proteins Celsr 1 and 3, Fzd 3, and Vangl 2 were depleted. They show that each cell develops translational polarization but cells in the vicinity are not polarized in the same direction (19). In this study, the rotational polarity was only affected when Celsr3 and Vangl2 were absent.

Although the core PCP pathway and cytoskeletal dynamics are probably the main mechanisms behind defining the direction of ciliary beating, ependymal polarity seems to be affected by additional parameters as well. For example, Matsuo et al. showed that disruption of ciliary motility affects both the tracheal and ependymal cilia but interestingly, the rotational polarity 
is only perturbed in the ependyma (77). However, once the ciliary beating direction is determined, a change of polarization and thus beating direction is not expected.

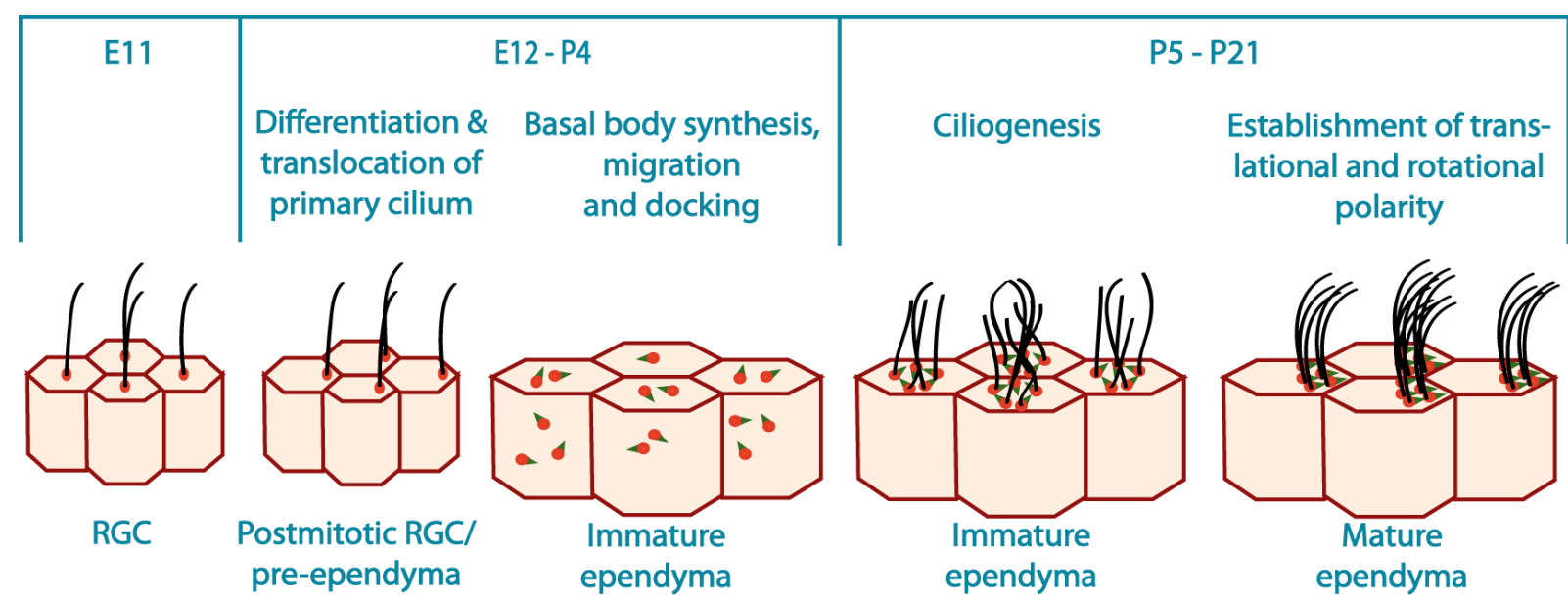

Fig. 9: Schematic illustration of ependymal development in the lateral ventricle. At E11, RGC have a centrally located primary cilium. Between E12-P4, all RGC differentiate into pre-ependymal cells and basal bodies are generated de novo to migrate and dock at the apical surface. Immature ependymal cells then start to form motile cilia, which in the course of maturation get translocated to one side of the cell and are aligned to one another. Cilia (black lines), basal bodies (red dots), basal feet (green triangle).

\subsection{Possible changes in flow pattern due to circadian rhythm}

When the complex flow pattern of the $\mathrm{v} 3 \mathrm{~V}$ was discovered, the authors also reported whirl formation at a particular site of the $\mathrm{v} 3 \mathrm{~V}$. They proposed that the whirl formation may be regulated by the mechanisms that govern the regulation of the circadian rhythm (18). The circadian rhythm describes the cyclic activity and rest pattern of the earth, which takes place in a period length of about $24 \mathrm{~h}$. In organisms, this rhythmic behavior is regulated by the so called circadian clock, an endogenous biological system that is synchronized by the circadian rhythm of the earth. The master regulator of this system is the suprachiasmatic nucleus (SCN), one of the hypothalamic nuclei that is located on the basal side of the $\mathrm{v} 3 \mathrm{~V}$ wall (136). The neurons of this nucleus receive information on the external light and dark cycle through the retinohypothalamic tract (RHT) and function as a pacemaker of peripheral clocks (137). The key genes and their protein products that govern the circadian clock interact with each other in a transcriptional - translational loop (TTL) of activation and inhibition and thus control each other's expression. One of the genes of this TTL is Period 1-3 (Per1-3), which despite its role in the circadian clock regulation, was linked with other functions. 


\subsubsection{Period in mechanisms other than circadian clock}

More than a decade ago, it was shown that the circadian clock machinery affects the cell cycle in adult mice by regulating the expression of cell cycle control genes like weel. The authors showed a strong correlation between the expression of clock genes with the timing of cell cycle events (138). PER was later shown to interact with the RNA binding protein Nono (139). This interaction controls the expression of the cell cycle checkpoint gene p16-link4A in a circadian manner (140). P16-INK4A regulates cellular senescence, which is a state of cell division arrest after proliferation (141). PER and Nono were shown to bind to the promoter of p16-link4A and activate its transcription. Mice that lack Period, show a decreased expression of p16-link4A, which was postulated to decrease senescence in dividing cells. This was tested by wounding wild type and $\mathrm{Perl}^{-/-} \mathrm{Per}^{-/}$double mutant mice to observe wound healing. 20 days post wounding, wild type mice recovered both dermal and epidermal cells, whereas Period mutants, showed significant defects in wound healing. The dividing cells of these mice could only give rise to epidermal cells that were immature and dysfunctional. Additionally, their healing was impaired due to a chaotic organization of the granulation tissue underneath (140).

Although the above mentioned studies show the involvement of PER in cell cycle control in mammalian adults, there is no evidence for its involvement in the timing of postnatal developmental processes that may involve the timing of cell division. However, the Caenorhabditis elegans (C. elegans) orthologue of period, lin42, has been shown to be involved in the timing of such processes (142-145). During larval maturation, C. elegans goes through four stages of molting. Lin 42 regulates the heterochronic miRNA expression in these stages and its mutation leads to premature terminal differentiation of hypodermal seam cells $(143,146)$. 


\section{Materials and Methods}

\subsection{Buffers and Solutions}

\subsubsection{PBS and PBST}

To prepare a $10 \mathrm{x}$ PBS solution the ingredients mentioned in table 1 were added to $900 \mathrm{~mL}$ of distilled water. After mixing the solution, distilled water was added until the volume reached 1 L. This solution was diluted 1:10 in distilled water to obtain a $1 \times$ PBS solution. After the dilution, the $\mathrm{pH}$ is at around 7.4. Minor $\mathrm{pH}$ adjustments can be performed by adding $1 \mathrm{~N}$ HCL or $1 \mathrm{~N} \mathrm{NaOH}$ to decrease or increase the $\mathrm{pH}$, respectively.

PBST was prepared by adding $2 \mathrm{~mL}$ of Triton X-100 (Merck KGaA) to $998 \mathrm{~mL}$ of 1x PBS solution.

Table $110 \times$ PBS

$\begin{array}{lll}\text { Ingredients } & \text { Quantity } & \text { Company } \\ \mathrm{NaCl} & 80 \mathrm{~g} & \text { Merck KGaA } \\ \mathrm{KCl} & 2 \mathrm{~g} & \text { Merck KGaA } \\ \mathrm{Na}_{2} \mathrm{HPO}_{4} & 14.4 \mathrm{~g} & \text { Merck KGaA } \\ \mathrm{KH}_{2} \mathrm{PO}_{4} & 2.4 \mathrm{~g} & \text { Merck KGaA } \\ \text { Distilled water } & \text { Fill up to } 11 & \text { Millipore }\end{array}$

\subsection{Animals}

All experiments of this study were performed on wild type C57BL/6N male mice at either P2024, 10-12 weeks, 4 months, 6 months, 9 months, or 1.5 years of age. The mice were sacrificed by cervical dislocation and whole mounts of the $\mathrm{v} 3 \mathrm{~V}$ were isolated. All experiments were performed according to the German Law on Animal Welfare and were authorized by the Office for Consumer Protection and Food Safety of the State of Lower Saxony. 


\subsection{V3V preparation}

The v3V whole mounts were prepared by first isolating the brain, which was placed in a coronal brain matric (Plano $\mathrm{GmbH}$ ). To obtain a $3 \mathrm{~mm}$ section, the brain was adjusted in the matric so that the first razor blade was placed at the optical chiasm and the second razor blade $3 \mathrm{~mm}$ posterior from the first. Both blades were pressed simultaneously to cut the tissue with the least possible deformation. Subsequently, the coronal section was placed in a Petri dish containing DMEM 21063 medium (Merck KGaA) with the anterior part facing upwards. The v3V was isolated in an open book preparation under a stereomicroscope (Fig. 10). An electrolytically sharpened tungsten needle was utilized to make the first cut through the midline of the tissue. This cut must be performed carefully so that the needle does not touch the walls of the v3V. To obtain v3V walls of both hemispheres, additional cuts were made on either side of the first cut. Next, both walls were separated like opening a book leaving the v3V wall facing upwards.

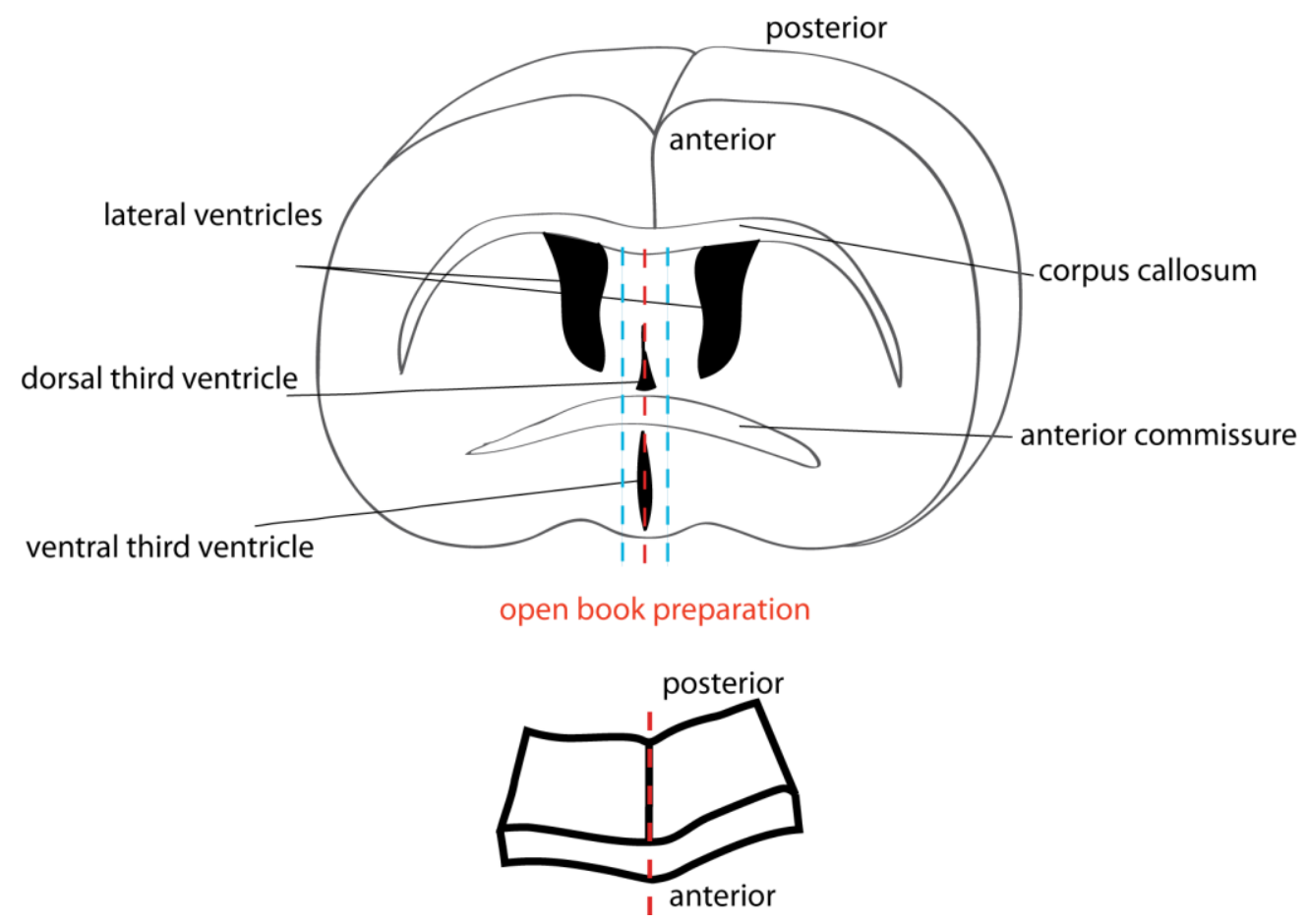

Fig.10: Schematic illustration of the $3 \mathrm{~mm}$ thick coronal section of a mouse brain. To obtain the left and right hemisphere ventral third ventricle $(\mathrm{v} 3 \mathrm{~V})$ walls, the first cut was made along the midline (dashed red line in the center). Parallel cuts were made left and right of the first cut (left and right dashed blue lines). Finally, the v3V was isolated and opened like a book with the cilia side facing upwards. 


\subsection{Fluorescent bead tracking and generation of flow maps}

The method of fluorescent bead tracking has been described previously (18). The resulting images of color-coded bead tracks were stitched together with a custom made graphical interface (GUI). For this, stationary beads were used as reference points in overlapping regions.

\subsection{Preparation of the four well plate}

The tissue was pinned on the surface of a four-well plate dish containing a layer of agarose (Biozym LE Agarose, Article number 840004). For this, a 2\% agarose solution was prepared in 1x PBS. This solution was added in the wells of a four well-plate leaving space for at least $500 \mu \mathrm{l}$ of volume for solutions. Once the agarose polymerizes, the four-well plate is ready to be used for the staining procedure.

\subsection{Slide preparation}

The tissue is mounted on a round slide (Asylum Research, 35 x $1 \mathrm{~mm}$ disk) to facilitate position correction for the tile scans. Furthermore, the prepared whole mount has a thickness of approximately $300 \mu \mathrm{m}$, therefore a round spacer with a thickness of $500 \mu \mathrm{m}$, with an overall diameter of $22.5 \mathrm{~mm}$ and an $8 \mathrm{~mm}$ inner pinhole was custom made and glued on the slide. To reduce the later distance of the sample to the microscope objective, low melting agarose (Agarose low melt, Roth, Article number 6351.1) was prepared and a drop was poured into the $8 \mathrm{~mm}$ pinhole of the spacer. A $2 \%$ solution was prepared and a painting brush was used to collect a drop and place into the $8 \mathrm{~mm}$ diameter pinhole of the spacer. After around $10 \mathrm{~min}$ at $4^{\circ} \mathrm{C}$, the low-melting agarose polymerizes and the slide can be used to for mounting. The mounting procedure is carried out by placing the tissue in the pinhole of the spacer.

\subsection{Immunohistochemistry}

The whole mounts were pinned to the previously prepared four-well plate while the ependymal cells were placed facing upwards. Then, the tissue was fixed in methanol for $10 \mathrm{~min}$ at $-20^{\circ} \mathrm{C}$. After two quick rinses with 1x PBS ( $\mathrm{pH} 7.4)$, the tissue was submerged in blocking solution 
(10\% goat serum in PBST (1x PBS; $\mathrm{pH} 7.4 ; 0.2 \%$ Triton $\mathrm{X})$ ) for one hour at room temperature. The primary antibody (Table 3) was diluted in blocking solution and incubated overnight at $4^{\circ} \mathrm{C}$. Subsequently, the whole mount was rinsed once and washed 4 times for 4 min in PBST. The secondary antibody (Table 4), diluted in blocking solution, was added and incubated for $1 \mathrm{~h}$ at room temperature at $70 \mathrm{rpm}$ on the shaker or overnight at $4^{\circ} \mathrm{C}$. After another washing step like described above, the tissue was mounted on a previously prepared slide in mounting medium (Vectashield H1500) and covered with a coverslip (Thermo scientific, 12mm \#1.5). The coverslip was gently pressed on the tissue and glued with commercially available nail polish. Before imaging, the tissue was kept at $4^{\circ} \mathrm{C}$ overnight to ensure the hardening of the mounting medium.

Table 2 Primary antibodies:

$\begin{array}{lll}\text { Antibody } & \text { Concentration } & \text { Catalog number and Company } \\ \gamma \text { tubulin (mouse) } & 1: 1000 & \text { T6557 Sigma } \\ \beta \text { catenin (mouse) } & 1: 2000 & \text { G10154 BD Biosciences } \\ \beta \text { catenin (rabbit) } & 1: 200 & \text { D10A8 Cell signaling } \\ \text { Vang11 (rabbit) } & 1: 1200 & \text { HPA025235 Sigma } \\ \text { Odf2 (rabbit) } & 1: 200 & \text { ab198189 abcam } \\ \text { Centriolin (mouse) } & 1: 1200 & \text { sc-365521 Sigma } \\ \text { Ki67 } & 1: 500 & \text { VP-RM04 Vector Laboratories }\end{array}$


Table 3 Secondary antibodies:

$\begin{array}{lll}\text { Antibody } & \text { Concentration } & \text { Catalog number and Company } \\ \text { Alexa488 anti mouse IgG1 } & 1: 400 & \text { A-21121 Invitrogen } \\ \text { Atto633 anti rabbit IgG } & 1: 300 & \text { 411036 Sigma } \\ \text { Alexa568 anti rabbit IgG } & 1: 300 & \text { A 11036 Thermo Fischer } \\ \text { Alexa594 anti rabbit IgG } & 1: 100 & \text { A-11012 Thermo Fischer } \\ \text { Abberior STAR RED anti } & 1: 100 & \text { Abberior Systems } \\ \text { mouse IgG } & & \\ \text { Abberior STAR580 anti rabbit } & 1: 100 & \text { Abberior Systems } \\ \text { IgG } & & \end{array}$

\subsection{Confocal Microscopy}

Tissue stained for $\beta$ catenin and $\gamma$ tubulin or Vangl1 was imaged using a Spinning disk confocal microscope (Olympus, Yokogawa). A single image covers a size of $136 \mu \mathrm{m}$ x $136 \mu \mathrm{m}$ (Andor iXon Ultra camera, chip size 512 x 512 pixel) and the ventricular surface was recorded in tiles with $20 \%$ overlap with a PlanApo N 60x/1.45 Oil (Olympus). 23 x 8 stacks were recorded per ventricular wall and the fluorescence was excited with $488 \mathrm{~nm}$ and $633 \mathrm{~nm}$ diode lasers (60 $\mathrm{mW}$ and $150 \mathrm{~mW}$, respectively; Phoxx series; Omicron) with an exposure time of $200 \mathrm{~ms}$.

Tissue stained for Odf2/centriolin was excited at $488 \mathrm{~nm}$ (Argon, $35 \mathrm{~mW}$ ) and $561 \mathrm{~nm}$ (DPSS, $20 \mathrm{~mW}$ ) and recorded by a point scanning confocal microscope with GaAsP-PMT. (Zeiss LSM880 with AiryScan) with a Plan-Apochromat 63x/1.4 Oil DIC M27 (Zeiss). One image has a size of $48.83 \mu \mathrm{m} \times 48.83 \mu \mathrm{m}(43 \mathrm{~nm} \times 43 \mathrm{~nm}$ per pixel). To record the largest area possible that could be stitched together, tile scans of z-stacks were acquired with a $10 \%$ overlap.

\subsection{STED Microscopy}

Super-resolution fluorescence microscopy (two-color Abberior STED 775 Quad scanning microscope, Abberior Instruments $\mathrm{GmbH}$ ) was performed for Odf2-centriolin stainings using a 
UPlanSapo 100xO/1.4. The STED high-power lasers $595 \mathrm{~nm}$ (up to $1000 \mathrm{~mW}$ ) and $775 \mathrm{~nm}$ (up to $3000 \mathrm{~mW}$ ) were used to excite the fluorescent signal of the specimen. The images were acquired with a widefield monochrome camera (1/2 inch CCD chip, 1280 x 960 pixels).

\subsection{Image segmentation and data analysis}

The following description of the algorithm, developed by Dr. Christian Westendorf using the MATLAB software, will focus on the main functions and principles and does not cover all details of the image binarization a subsequent data analysis.

\subsubsection{Translational polarity and $\mathrm{v} 3 \mathrm{~V}$ architecture analysis}

In order to determine the translational polarity directions of all cells in the $\mathrm{v} 3 \mathrm{~V}$, the $\mathrm{z}$-stacks of $\beta$ catenin (cell boundary) and $\gamma$ tubulin (basal body) staining were acquired by a confocal spinning disk microscopy. All stacks first underwent a flat field correction to rectify uneven illumination of the specimen. Then, every stack was maximum projected in order to obtain a 2D image of each 3D stack. Using the MATLAB routine normxcorr2, these images were then 2D cross-correlated and, the direct neighbors were aligned to one another and a stitched image was created. In this image, the overlapping regions are the average of all respective maximum intensity projections located in the overlap. Then, image smoothing was performed and the image was binarized by thresholding it via the k-means algorithm using the MATLAB internal k-means routine. Afterward, objects smaller than 10 pixels were removed from further analysis. The fluorescent signal of $\beta$ catenin was next enhanced by using a vesselness filter (147). The segmentation of each channel's signal was carried out with a marker based watershed transformation, where the $\gamma$ tubulin mask acted as the marker and the $\beta$ catenin as the watershed. The resulting binarized mask enabled the computation of the translational polarity that is defined as the vector from the centroid of the cell to the cilia bundle $(\overrightarrow{\boldsymbol{C C}}$ vector $)$. The angle of this vector is then calculated with respect to the image coordinates. The length of this vector was used to determine the degree of translational polarization. Furthermore, this segmentation also enabled the computation of the area that is surrounded by $\beta$ catenin (apical surface area/ cell size) and $\gamma$ tubulin (size of cilia bundle). Moreover, we determined the eccentricity of each cilia bundle by using the MATLAB routine regionprops. For the eccentricity, the value 0 depicts a circular bundle, while 1 represented a cilia bundle in the shape of a line. 
Tissue stained for Vangl1 one was also acquired with a spinning disk confocal microscope. The same image processing algorithms were used as described above, except that the signal of Vangl1 was not subject to binarization and subsequent analysis.

\subsubsection{Human verification of analysis method}

The $\overrightarrow{\boldsymbol{C C}}$ vectors that were calculated by our algorithm were next compared with vectors that were drawn manually using a custom made graphical user interface (GUI). Such vectors were annotated for 50 cells in 3 animals. While the human eye can recognize every cell and designate a translational polarity vector to it, the computer may not always recognize a cell and its boundaries. In such a case, no $\overrightarrow{\boldsymbol{C C}}$ vector is drawn by the computer. On average, the manually drawn vectors and those defined by our algorithm deviate by less than $15^{\circ}$ in angle.

\subsubsection{Rotational polarity analysis}

To determine the rotational polarity direction of each cilium of a cilia bundle (Odf 2 and centriolin), z-stacks. These z-stacks were first stitched and maximum projected by using the Zeiss Zen black software 2.3 SP1. Then, the images were segmented into fore- and background by kmeans clustering. Next, the watershed transformation was used to distinguish individual cilia bundles. Subsequently, the signals of Odf2 and centriolin were dilated, so that additional pixels were added to each signal and ensured an overlap between objects. This way the program recognizes individual cilia bundles. Then, local intensity maxima of the Odf 2 and centriolin signals were computed using the MATLAB routine imregionalmax. The program next searched for overlaps or closest proximity between Odf2 and centriolin signal in order to detect the most likely pairs of the basal body and basal foot. It is important to note that with this imaging method we reach the resolution limit and due to the structure of basal bodies and basal feet, each signal can have multiple local intensity maxima. Finally, the directions of all resulting vectors were averaged into one vector that depicts the rotational polarity of a cilia bundle. 


\subsubsection{Human verification of analysis method}

The accuracy of our algorithm was tested by manually annotating vectors from the basal bodies to their respective basal feet using a custom made GUI using MATLAB. This annotation was performed for 655 basal body and basal foot pairs in one sample, 689 pairs in a second sample and 724 pairs in a third sample that are part of $20-25$ cilia bundles per sample. An average of these manually drawn vectors was then calculated for each cilia bundle, which displays the beating direction of a cilia bundle. Finally, we computed the deviation between the human and computer-generated directions of each bundle's rotational polarity. 


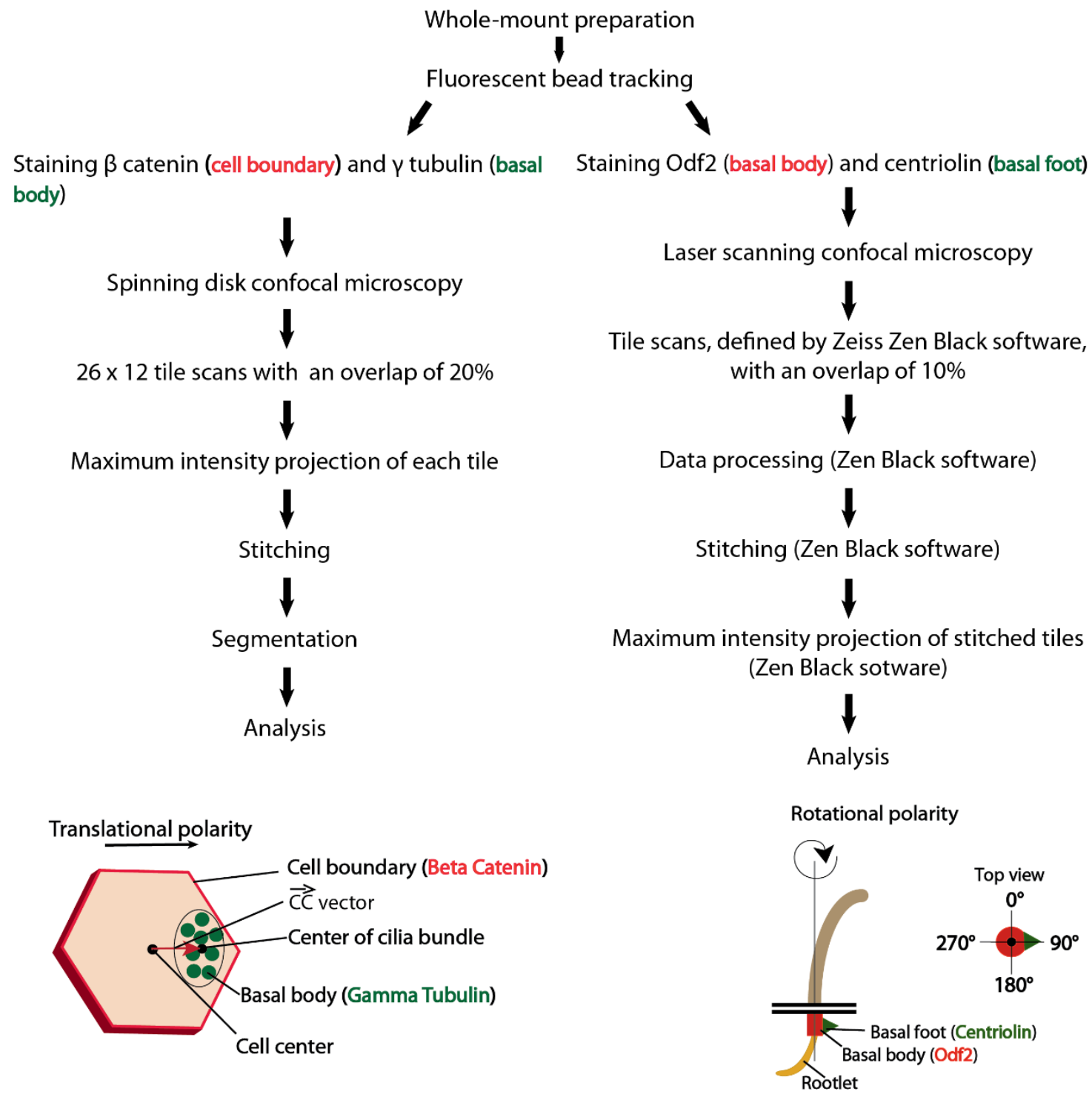

Fig.11: Summary of both the experimental and data analysis methods to evaluate translational and rotational polarity of $\mathrm{v} 3 \mathrm{~V}$ ependymal cells. 


\section{Results}

\subsection{Planar cell polarity establishes ciliary beating direction}

In tissues such as the Xenopus larval skin, mammalian trachea, or the oviduct, where the beating of motile cilia is unidirectional, the cilia bundles of all cells beat in the same direction $(113,148,149)$. In contrast, the near-wall flow pattern of the v3V is complex, containing straight, bent, opposing and whirl shaped flows. Furthermore, this flow pattern is dynamic and can change its direction, namely in the case of whirls that occur in the center of the v3V (18). We investigated if the complex pattern, observed in explants, is determined by PCP signaling and would therefore, resemble the in vivo near-wall flow. For this, we checked the translational polarity, the expression of the core PCP protein Vangl 1, and the rotational polarity in all areas of the $\mathrm{v} 3 \mathrm{~V}$.

\subsubsection{Translational polarity correlates with the v3V flow-pattern}

To investigate how the translational polarity is correlated with the flow pattern in the v $3 \mathrm{~V}$, we first prepared $\mathrm{v} 3 \mathrm{~V}$ whole mounts from a freshly dissected brain and submerged it in medium containing homogeneously dispersed fluorescent latex-beads. The bead movement near the ependymal wall that is caused by ciliary beating was recorded and tracked to create $\mathrm{v} 3 \mathrm{~V}$ flow maps as described previously (18). Since the flow pattern shows nine different directionalities of bead movement, we subdivided the v3V into nine modules (Fig. $12 \mathrm{~A}$ ). It should be noted that we subdivided the modules in a slightly different manner that the subdivision carried out by Faubel et al., 2016. The changes we made in dividing the modules were in modules 1, 2 and 6. Module 1 is marked as the area, where the CSF enters the $\mathrm{v} 3 \mathrm{~V}$ at the foramen of Monro and merges into module 3. Module two is located ventral to module one, in which anteriorly the flow fans out towards the anterior $\mathrm{v} 3 \mathrm{~V}$ boundary but is posteriorly directed towards $100^{\circ}$ to $200^{\circ}$ (according to the legend in Fig. 12 A) and joins module 8. Module 3 and 4 are located near the dorsal center, where their flow patterns oppose one another and form the so-called separatrix (18). The whirl at this location will be addressed as the central whirl. The area, where whirls occur most consistently, is ventral from the separatrix. The flow in the fifth module is a continuation of the flow of module 3. It is flow direction is in a wave pattern and leads towards the ventral boundary of the v3V. Module 6 is further subdivided in $6 \mathrm{a}$ and $6 \mathrm{~b}$. In module $6 \mathrm{a}$, the flow starts posterior of module 4 at the dorsal $v 3 \mathrm{~V}$ boundary and is oriented towards module 
$6 \mathrm{~b}$ with flow directions ranging from to $200^{\circ}$ to $260^{\circ}$. Module $6 \mathrm{~b}$ starts where the flow of module 6a makes a turn in the direction of $225^{\circ}$ to meet module five and eight at the center of the ventral edge of the $\mathrm{v} 3 \mathrm{~V}$. The flow of this module starts at the posterior end of the $\mathrm{v} 3 \mathrm{~V}$ and is directed towards module 5 and 8 . The CSF leaves the v3V along module 7, where it is directed from module 6a towards the aqueduct of Sylvius. Module 8 lies in the anterior-ventral region, bordering module 2, 5 and $6 \mathrm{~b}$. The flow of module 8 leads from the anterior ventral region of the $\mathrm{v} 3 \mathrm{~V}$ towards the separatrix.

Posterior to module $6 \mathrm{~b}$ and ventral to module 7 , no near-wall flow was detected. This area of the $\mathrm{v} 3 \mathrm{~V}$ is made of with tanycytes, which possess 1-2 immotile primary cilia and do not contribute to the $\mathrm{v} 3 \mathrm{~V}$ flow in our measurement (Fig.12 A). Although we cannot rule out that in a living organism the tanycyte region may contribute to the $\mathrm{v} 3 \mathrm{~V}$ bulk flow through fluid transport from the brain parenchyma into the ventricle or vice versa $(7,150)$.

After having established the flows above the explant, the same tissue was stained for the adherence junction (cell boundary) marker, $\beta$ catenin, and basal body marker, $\gamma$ tubulin, to determine the translational polarity of cells in all $\mathrm{v} 3 \mathrm{~V}$ modules. The ventricular surface was recorded in tiles $(136.5 \mu \mathrm{m} \times 136.5 \mu \mathrm{m})$ with a $20 \%$ overlap. Per ventricular wall, $184 \mathrm{z}$-stacks (23 x 8$)$ were recorded and stitched together (Fig.12 B).

Next, we used the algorithm, established by Dr. Christian Westendorfs, that segmented the fluorescent signal of $\beta$ catenin to calculate the cell centroid and $\gamma$ tubulin to determine the cilia bundle centroid, the connection of which determines the $\overrightarrow{C C}$ (Fig.12 C, D, E). The direction of the $\overrightarrow{C C}$ vector depicts the translational polarity direction, which was then calculated for around 20000 thousand cells covering all v3V modules. To visualize the $\overrightarrow{C C}$ direction of each cell we used the same color code as for the bead track directions in the flow-maps and generated a plot, in which the direction of translational polarization (TP) of each cell is recognizable by its color (Fig. 12 F).

TP plots were next divided in modules, in which all cells within a module posses a similar direction of translational polarity. On all TP plots, we could identify nine modules, which show the same pattern as their corresponding flow map generated by bead tracking. With circular plots, we quantified the directions of the bead tracks and of the translational polarity for each module in three mice (Fig.13, 14). It should be recalled that the bead track directions are derived from tracs of fluorescent beads, which move near the ventricular wall due to cilia beating. The recording of these moving beads occurs on several planes above the cilia since the v $3 \mathrm{~V}$ surface 
on the whole mount does not lie flat but covers up $100 \mu \mathrm{m}$ in the z-axis. In the resulting flowmaps, the tracks of these planes are averaged and give a general direction of flow in a particular area. This means that the range of flow directions and translational polarity direction cannot match exactly. Nonetheless, we observe a pattern of translational polarity directions that varies little among animals and correlates with the general flow pattern of the v3V (Fig. 13, 14). In module one, the bead tracks directionality ranges from $110^{\circ}$ to $190^{\circ}$ while the translational polarity direction ranges from $60^{\circ}$ to $230^{\circ}$. In the second module, the bead tracks range from $190^{\circ}$ to $300^{\circ}$ and the translational polarity directions from $100^{\circ}$ to $260^{\circ}$. The third and fourth module, oppose one another, where the bead tracks range from $90^{\circ}$ to $180^{\circ}$ and $200^{\circ}$ to $320^{\circ}$, respectively. The translational polarity directions of the third and fourth module range from $40^{\circ}$ to $180^{\circ}$ and $160^{\circ}$ to $330^{\circ}$, respectively. In module 5 , the flow directions range from $60^{\circ}$ to $230^{\circ}$ and the translational polarity directions from $0^{\circ}$ to $270^{\circ}$. This is a region, where the flow pattern is either in a waveform and/or has a whirl. Additionally, modules 3, 4, 6, and 8 border this module. Therefore, a high range in flow and especially translational polarity directions of this region is expected. Module 6a further reflects the flow pattern, in which the bead tracks range from $100^{\circ}$ to $220^{\circ}$ and the translational polarity directions from $50^{\circ}$ to $190^{\circ}$. In all observed animals, the flow pattern of module $6 \mathrm{~b}$ and the translational polarity of the cells in this region do not correlate in the same manner as in the other modules. While the bead tracks range from $200^{\circ}$ to $270^{\circ}$, the translational polarity ranges from $60^{\circ}$ to $270^{\circ}$. Finally, the translational polarity directions of modules 7 and 8 correlate with the flow pattern directions. The bead track directionality ranges from $320^{\circ}$ to $80^{\circ}$, and $40^{\circ}$ to $110^{\circ}$, whereas the translational polarity ranges from $240^{\circ}$ to $100^{\circ}$ and $350^{\circ}$ to $130^{\circ}$, respectively (Fig. 13, 14). 

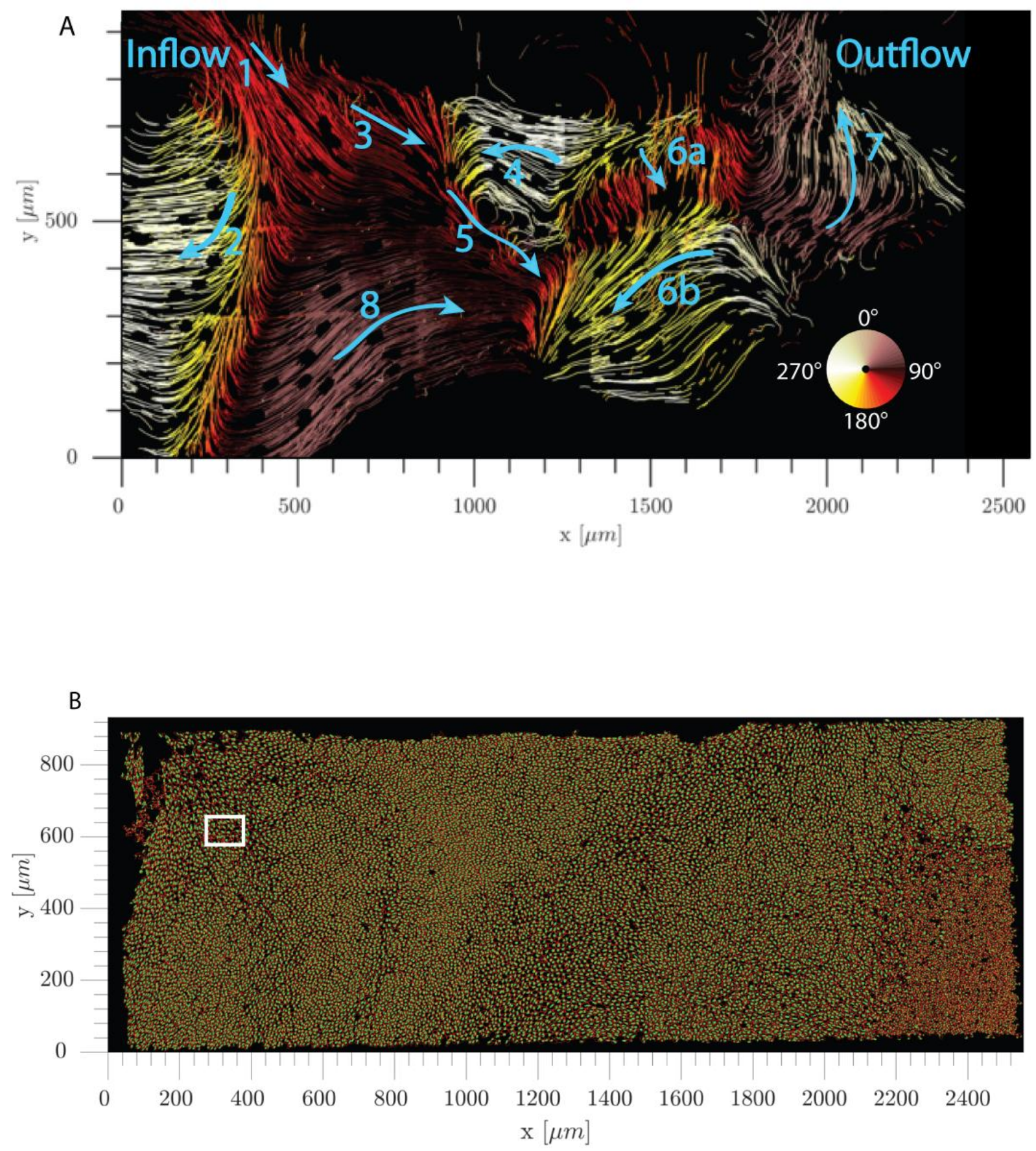

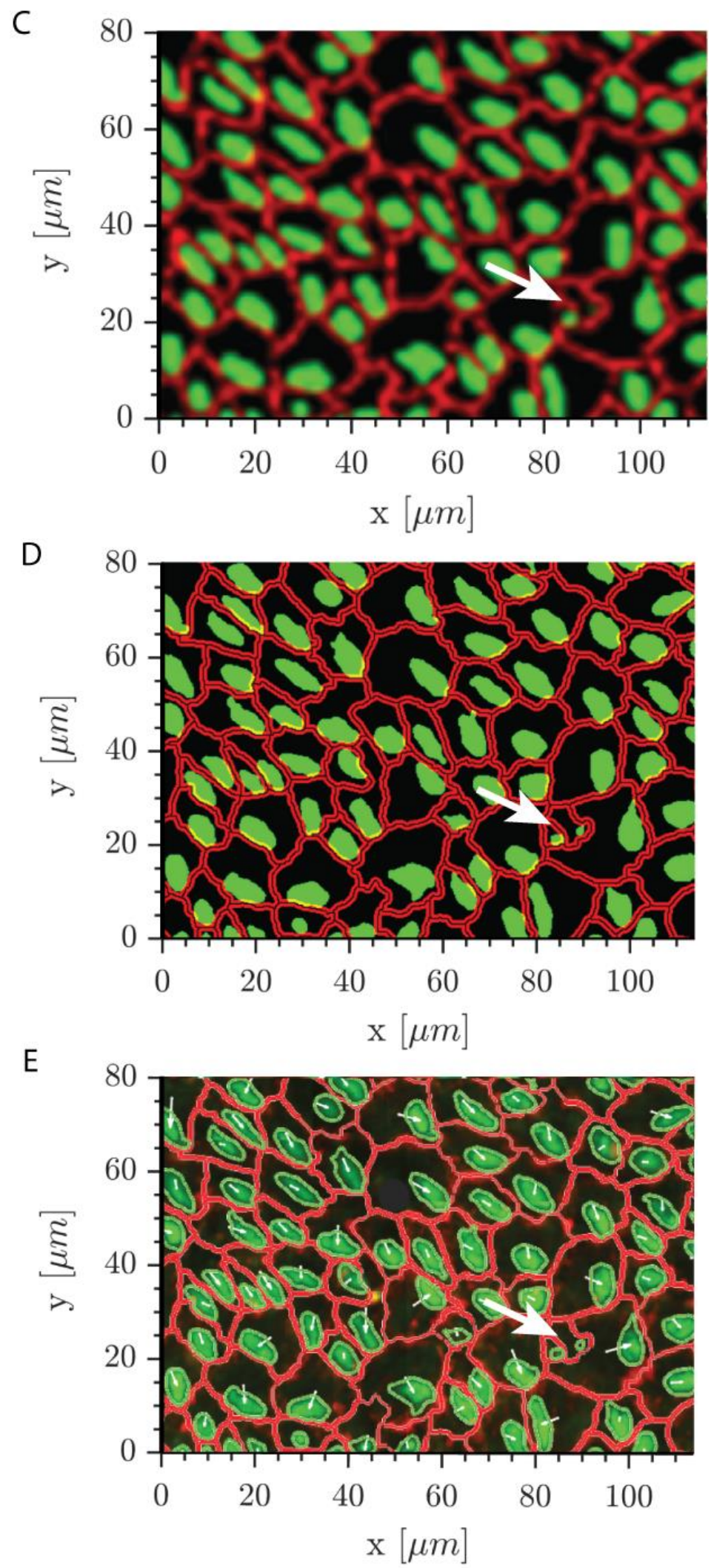


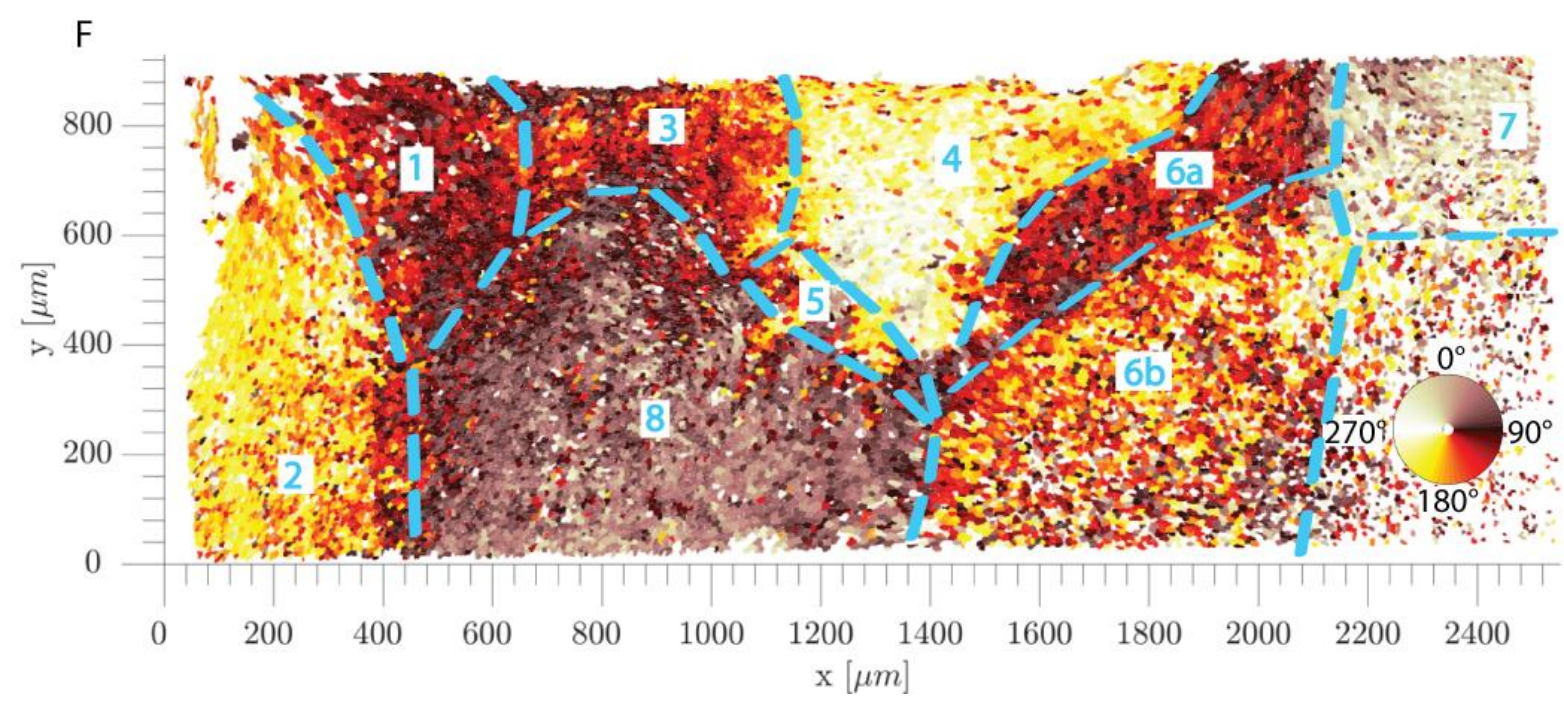

Fig. 12: The v3V pattern of translational polarity directions correlates with the flow pattern. (A) Flow-map of a v3V whole mount, where blue arrows indicate the general flow direction within the respective module. (B) Maximum intensity projected and stitched image of the same $\mathrm{v} 3 \mathrm{~V}$ whole mount with $\beta$ catenin (red) and $\gamma$ tubulin (green) staining. A white square indicates the enlarged region shown in $\mathrm{C}$, where multiciliated ependymal cells and two monociliated B1 (large white arrow) cells can be seen. (D, E) Segmentation of the $\beta$ catenin and $\gamma$ tubulin signal to calculate the $\overrightarrow{\boldsymbol{C C}}$ vector (small white arrows), connecting the cell centroid with the centroid of the cilia bundle. (F) V3V plot that shows translational polarity across the entire tissue. To visualize the $\overrightarrow{\boldsymbol{C C}}$ direction of each cell we used the same color code as for the bead track directions (legend on the lower right). The translational polarity presages the overall flow pattern. The only exception is module $6 \mathrm{~b}$. 

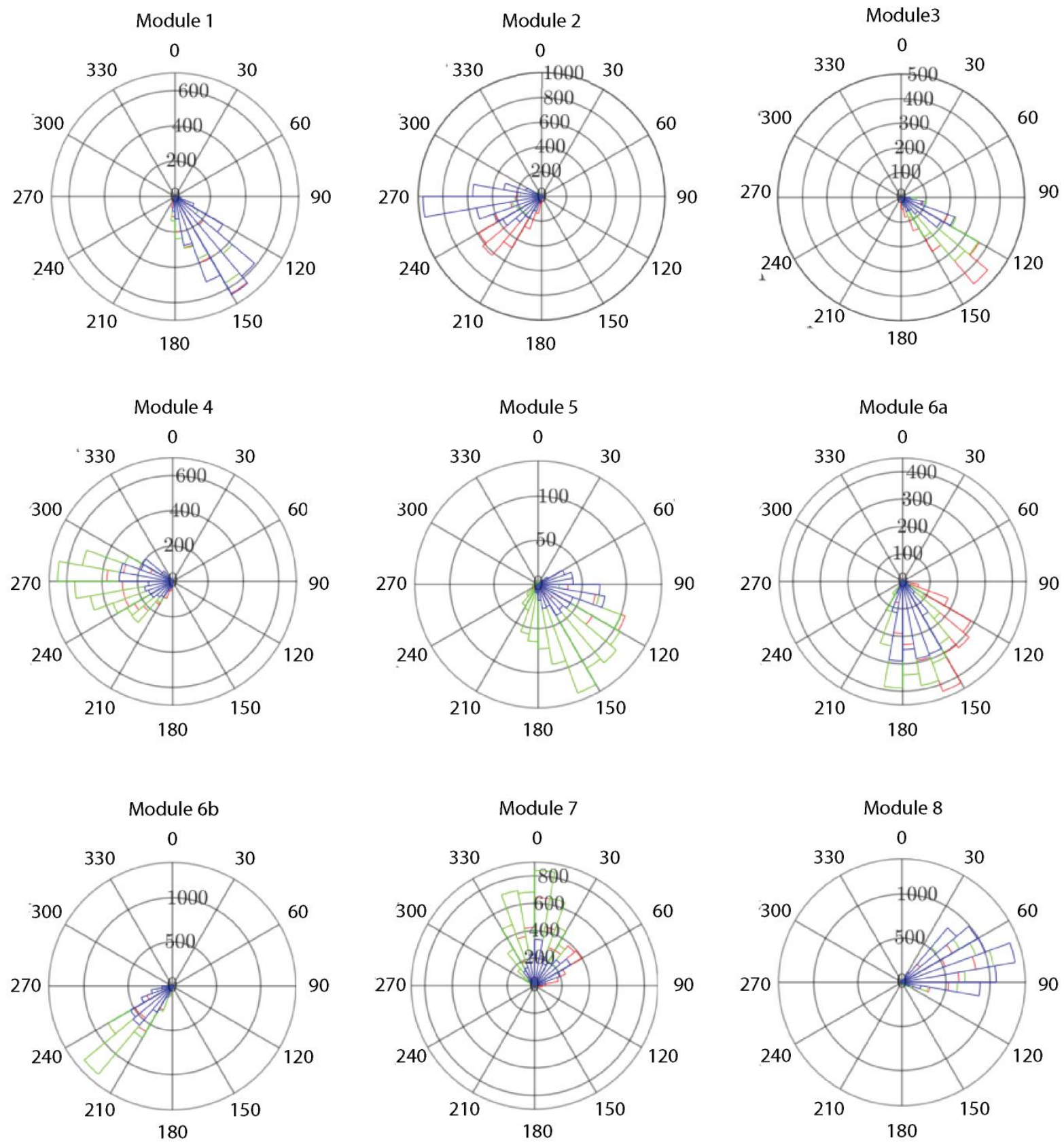

Animal 1
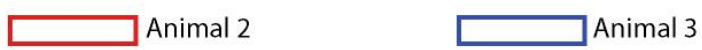

Fig. 13: Circular plots of bead track directions in each module. For three wild type mice (red, green, blue bars), circular plots show the quantification of the bead track directions in each module. In all mice, the tracks of beads show similar ranges of directionalities. 

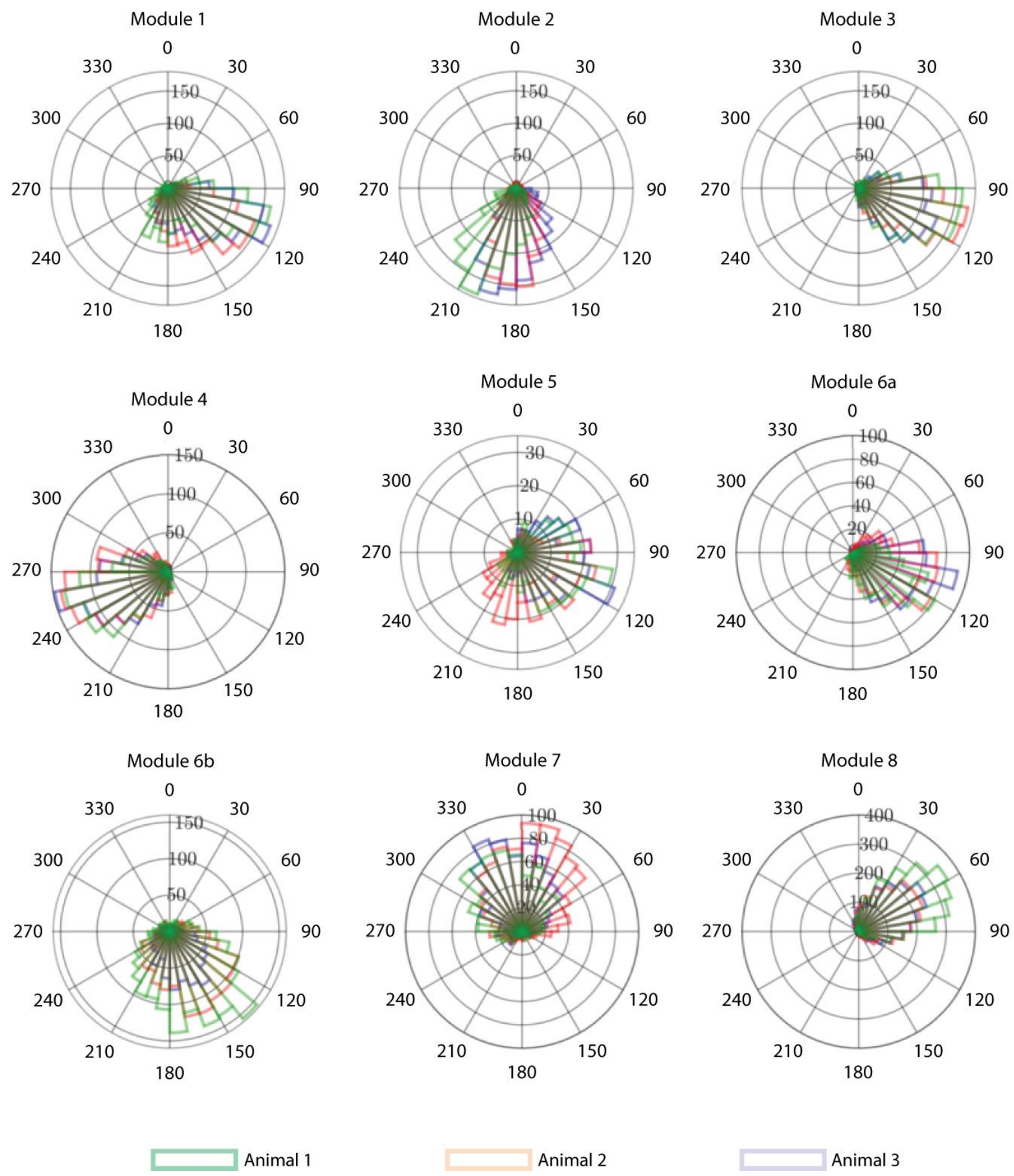

Fig. 14: For three wild type mice (green, orange, purple bars), circular plots show the quantification of translational polarity directions of all cells within each module. In all mice, the translational polarity directions in each module ranges in similar directionalities. 
The $\overrightarrow{\boldsymbol{C C}}$ vectors that were calculated by our algorithm were next compared with vectors that were drawn manually using a graphical user interface (GUI). Vectors from the cell centroid to the cilia bundle centroid were annotated for 50 cells in 3 animals. While the human eye can recognize every cell and designate a translational polarity vector to it, the computer may not always recognize a cell and its boundaries. In such a case, no $\overrightarrow{\boldsymbol{C C}}$ vector is drawn by the computer. On average, the manually drawn vectors and those defined by our algorithm deviate between $5^{\circ}$ to $20^{\circ}$ in angle (Fig. 15).

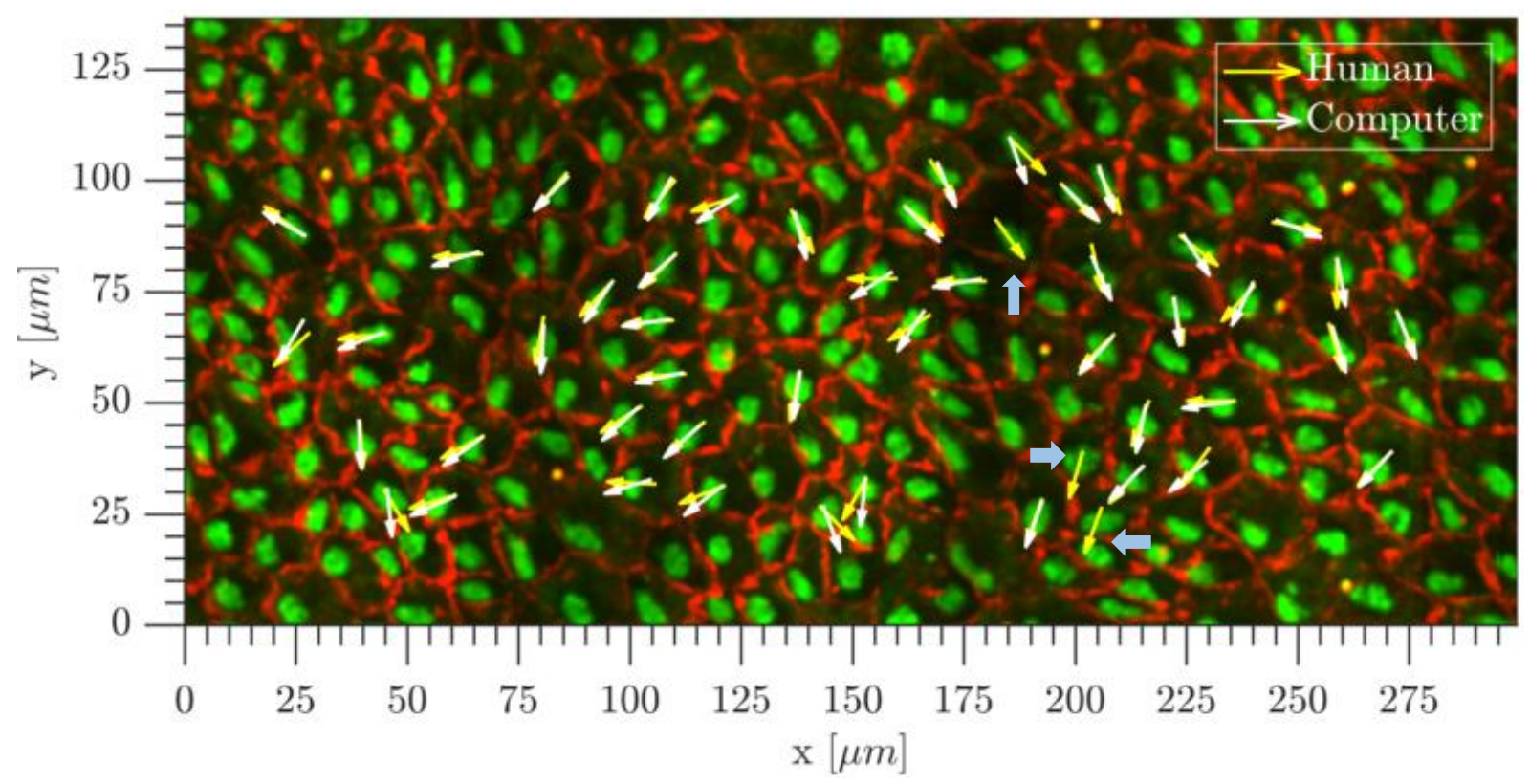

Fig. 15: Comparison of human annotated and computer calculated $\overrightarrow{\boldsymbol{C C}}$ vectors on ependymal cells. The vectors calculated by our algorithm are in white, while those that were human annotated are shown in yellow. For three human annotated cells in this sample, the computer did not recognize a cell and thus did not designate a $\overrightarrow{\boldsymbol{C C}}$ vector (blue arrows).

We conclude that the complex flow pattern of the $\mathrm{v} 3 \mathrm{~V}$ is directly correlated with a complex pattern of translational polarity directions. 


\subsubsection{Vangl 1 expression varies in different $v 3 \mathrm{~V}$ regions}

We next ought to investigate how PCP proteins are localized in the v3V ependyma, for which we first generated a flow-map of the v3V whole mount (Fig. $16 \mathrm{~A}$ ) and then stained the same tissue for Vangl 1, which is one of the commonly used markers for PCP $(109,151)$. Vangl 1 is a transmembrane protein that is typically expressed in a chevron/arrowhead shape and is located on the opposite side of the cilia bundle. An arrowhead like shape of Vangl 1 expression indicates that the cilia beat in the opposite direction to which the arrowhead is pointing (109). Our method of first recording the flow pattern of the $\mathrm{v} 3 \mathrm{~V}$ and then staining the entire $\mathrm{v} 3 \mathrm{~V}$ of the same specimen for Vangl 1, enables a correlation of flow direction with Vangl 1 expression pattern. The representative images of the Vangl 1 expression in straight flows of the $\mathrm{v} 3 \mathrm{~V}$ for modules 1, 2, 6b, and 7 of the same sample show how within each module, Vangl 1 has the typical chevron-like pattern, indicating aligned polarization within modules (Fig. 16 B, C, F, G). Furthermore, the directionality of the Vangl 1 pattern correlates with the flow direction of the respective modules. The cells in the area of a whirl or junctions of 2 opposing flows are not expected to have the same direction of polarization and are thus not expected to show a chevronlike pattern. We confirmed that in these regions, Vangl 1 expression pattern is distorted and indicates a lack of alignment in adjacent cells (Fig. 16 DE).

These results indicate that PCP aligns tissue polarity within each module. In addition, interdomain directionalities of cellular polarity vary in the same manner as the flow pattern and the translational polarity. As expected, in areas such as whirls or the borders of modules, where the flows with different directionalities come together, Vangl 1 expression is complex. The question arises whether the cilia of these cells beat in a polarized manner. In the next chapter we will show, how the rotational polarity determines the polarized beating of cilia in these areas. 


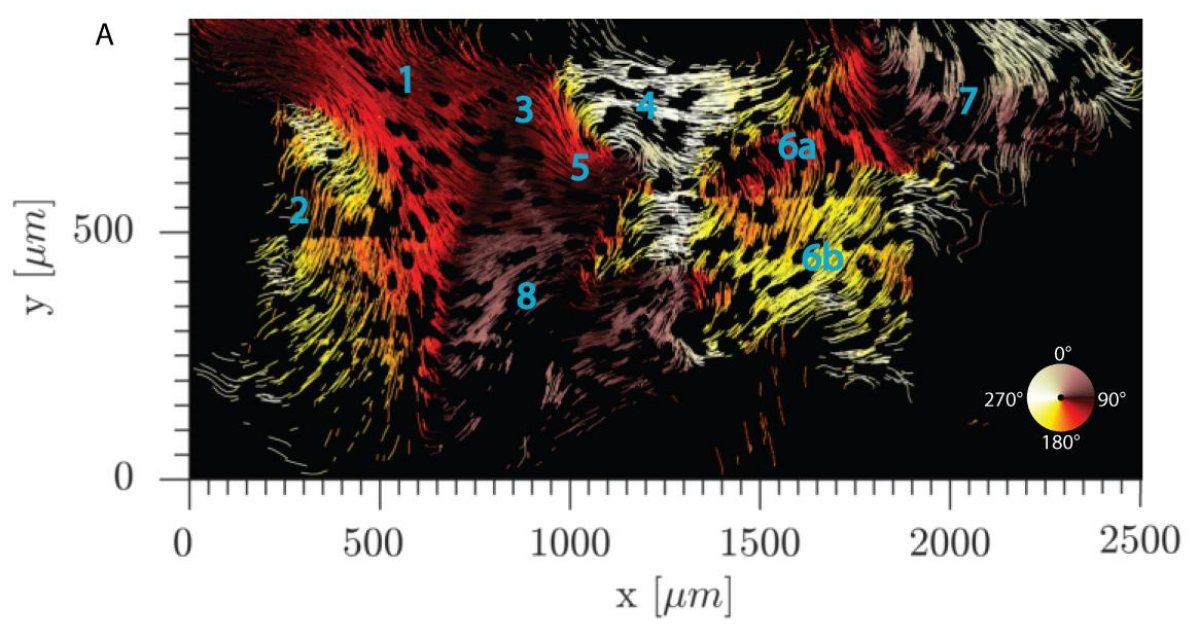

B
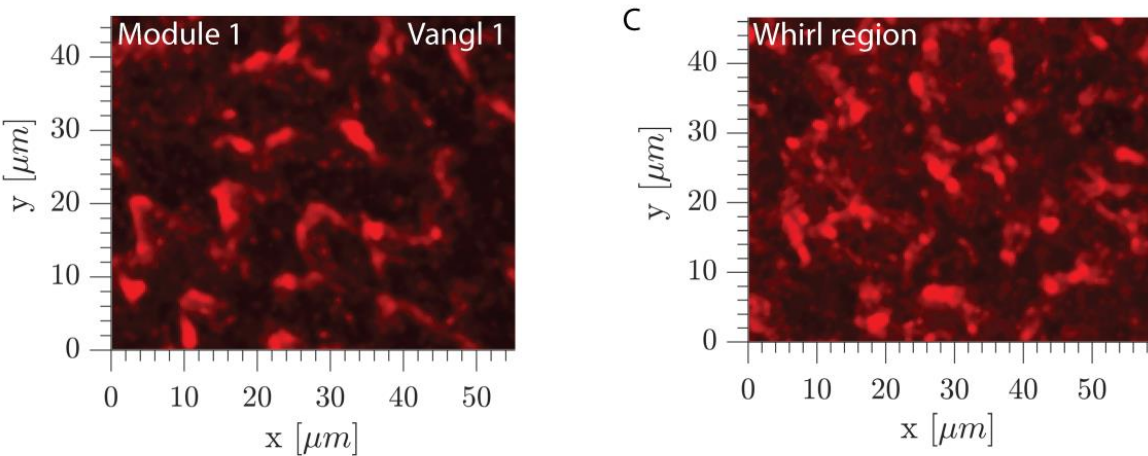

D

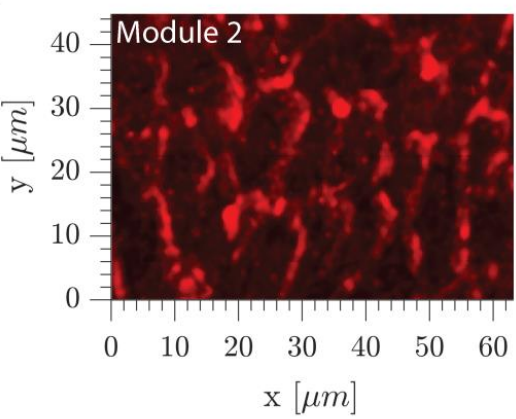

E

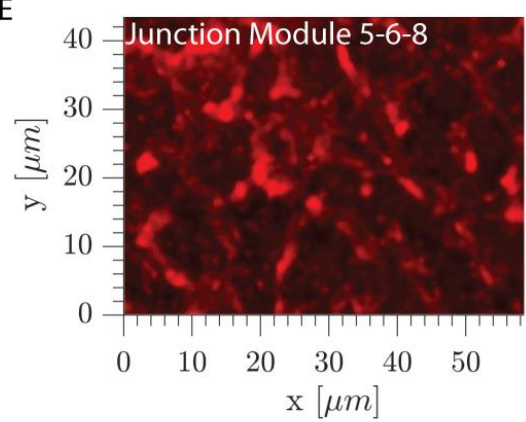

F

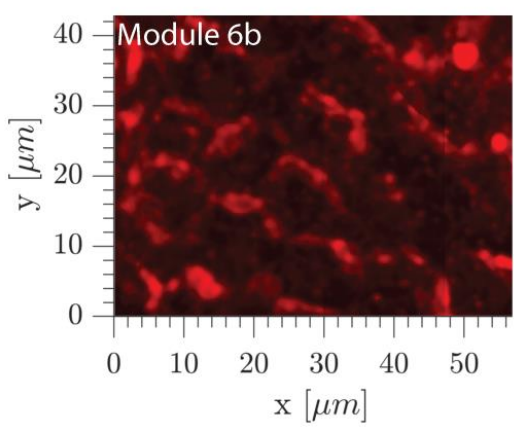

G

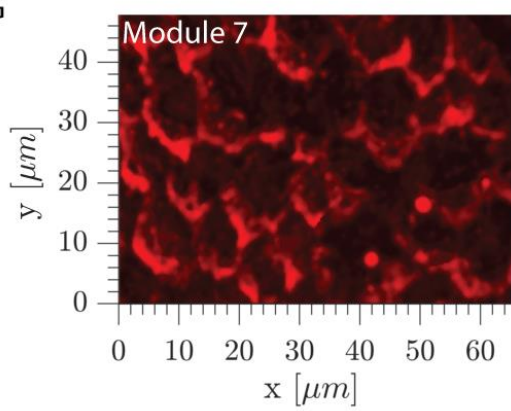

Fig. 16: Vangl 1 expression correlates with the v3V flow pattern. (A) V3V flow-map showing all modules with different flow-directions (Module numbers in blue). Representative images showing Vangl 1 expression in modules 1 (B), whirl region (C), 2 (D), junction of module 5,6, and 8 (E), module $6 \mathrm{~b}(\mathrm{~F})$, and module $7(\mathrm{G})$. Note that Vangl 1 expression in a straight flow shows a chevron pattern, whereas cells in the whirl area or at junctions of opposing flows exhibit a distorted pattern. 


\subsubsection{The rotational polarity presages the flow-pattern of ependymal cilia}

We have shown that cells within straight flows display a clear translational polarity and a chevron-like Vangl 1 expression. However, the cells that create a whirl possess motile cilia with directional beating, although they do not display whirl shaped translational polarities or polarized Vangl 1 expression in adjacent cells. This prompted us to analyze the polarization of each cilium, by localizing the position of the basal foot relative to the basal body. The basal foot connects the ciliary base to the cytoskeleton and its polarized position at the basal body correlates with the cilium's beating direction $(116,152)$.

First, we stained the basal body with Odf2 and the basal foot with centriolin, as illustrated in figure 8 (117). Using STED microscopy, we confirmed that all cilia within a bundle have approximately the same rotational polarity (Fig. 17 A). Then, another sample stained for Odf2 and centriolin was checked for the rotational polarities via laser - scanning microscopy. Maximum intensity projected stacks from this sample also show that all cilia in a bundle possess a similar rotational polarity direction (Fig. 17 B). Additionally, we found that every cilia bundle contains 1-2 basal bodies that have multiple basal feet, which may be primary cilia (Fig. 17). For further evaluation, we chose laser - scanning microscopy, since STED microscopy does not allow the acquisition of a larger region of interest. 

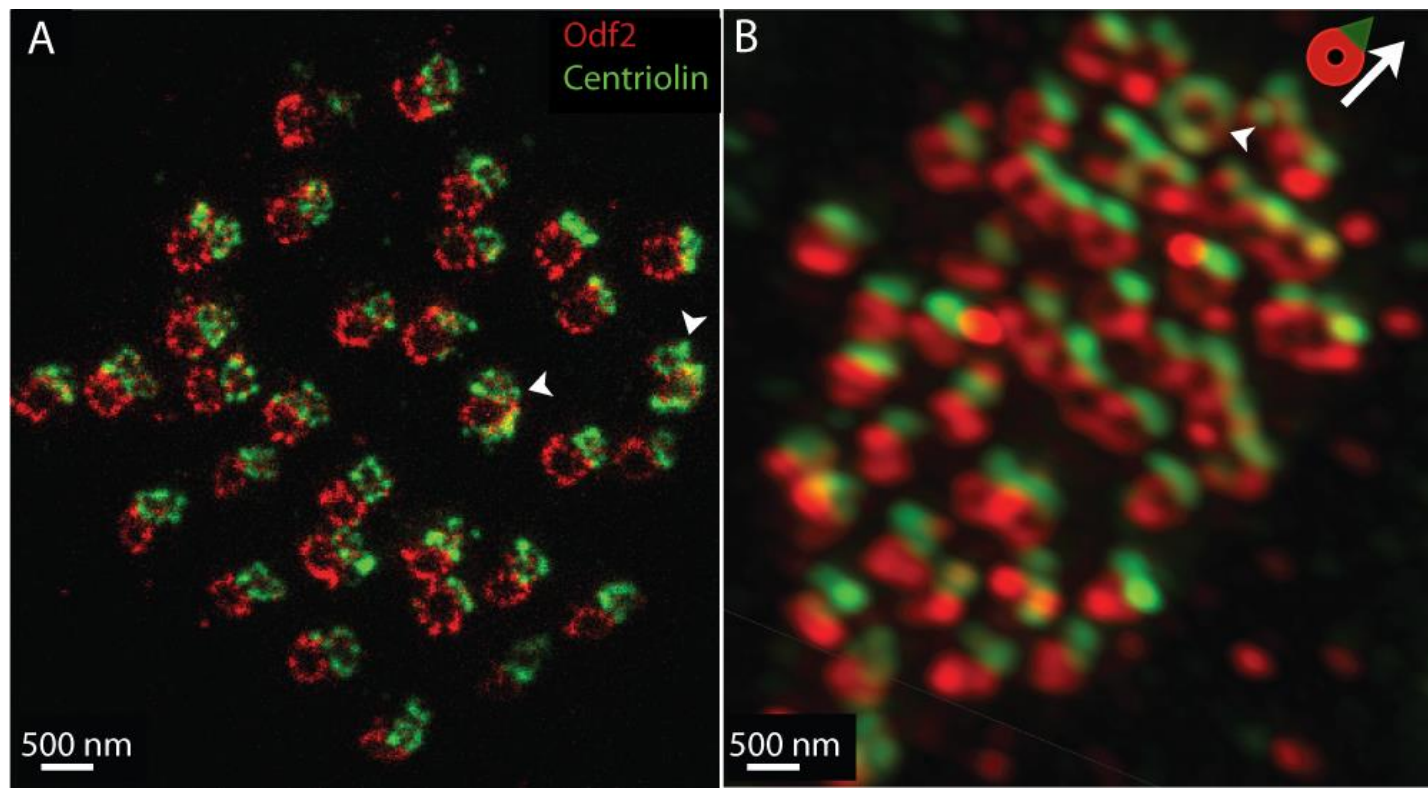

Fig. 17: Rotational polarity of all cilia in a bundle is visualized by staining the basal body with Odf2 (red) and basal foot with centriolin (green). (A) STED imaging slice of neighboring cilia within one bundle. (B) Maximum intensity projected laser scanning microscopy image of all cilia in a bundle. In both cases, within each bundle, the rotational polarity of individual cilia is approximately the same. A white arrow indicates the average rotational polarity direction of the shown cilia bundle. Basal bodies that show a centriolin signal on multiple sites are marked with an arrowhead. 1-2 such cilia are observed in every cilia bundle.

To calculate the rotational polarity of a larger area, we chose laser - scanning microscopy for further investigation. For the determination of the rotational polarity of each cilium, the algorithm, written by Dr. Christian Westendorf, computed local intensity maxima of the Odf2 and centriolin signals. The program next searched for overlaps or closest proximity between the fluorescent signal of Odf 2 and centriolin in order to detect the most likely pair of basal body and basal foot. It is important to note that with this imaging method we reach the resolution limit of light microscopy and due to the structure of basal bodies and basal feet, each signal can have multiple local intensity maxima. Subsequently, the directions of all resulting vectors were averaged into one vector that depicts the average rotational polarity of a cilia bundle (Fig. 18).

The accuracy of our algorithm was tested by manually annotating vectors from the basal bodies to their respective basal feet. We annotated vectors for 655 basal body and basal foot pairs in one sample, 689 pairs in a second sample and 724 pairs in a third sample that are part of $20-$ 25 cilia bundles per sample. An average of these manually drawn vectors was then calculated for each cilia bundle, which displays the beating direction of a cilia bundle (Fig. 18 A). The human and computer-generated directions of each bundle's rotational polarity deviated by $9.8^{\circ}$ 
in the first, $6.8^{\circ}$ in the second, and $11.1^{\circ}$ in the third sample. While the human eye is able to recognize a basal body and basal foot pair despite the limited resolution of the image, the computer calculates intensity maxima of the fluorescent signal of Odf 2 and centriolin to draw a vector. Since each basal body and basal foot pair can have multiple intensity maxima due to the shape of these structures, each pair is often designated more than one vector. This results in an average vector per cilia bundle that deviates from that calculated from human annotations (Fig.18 B).

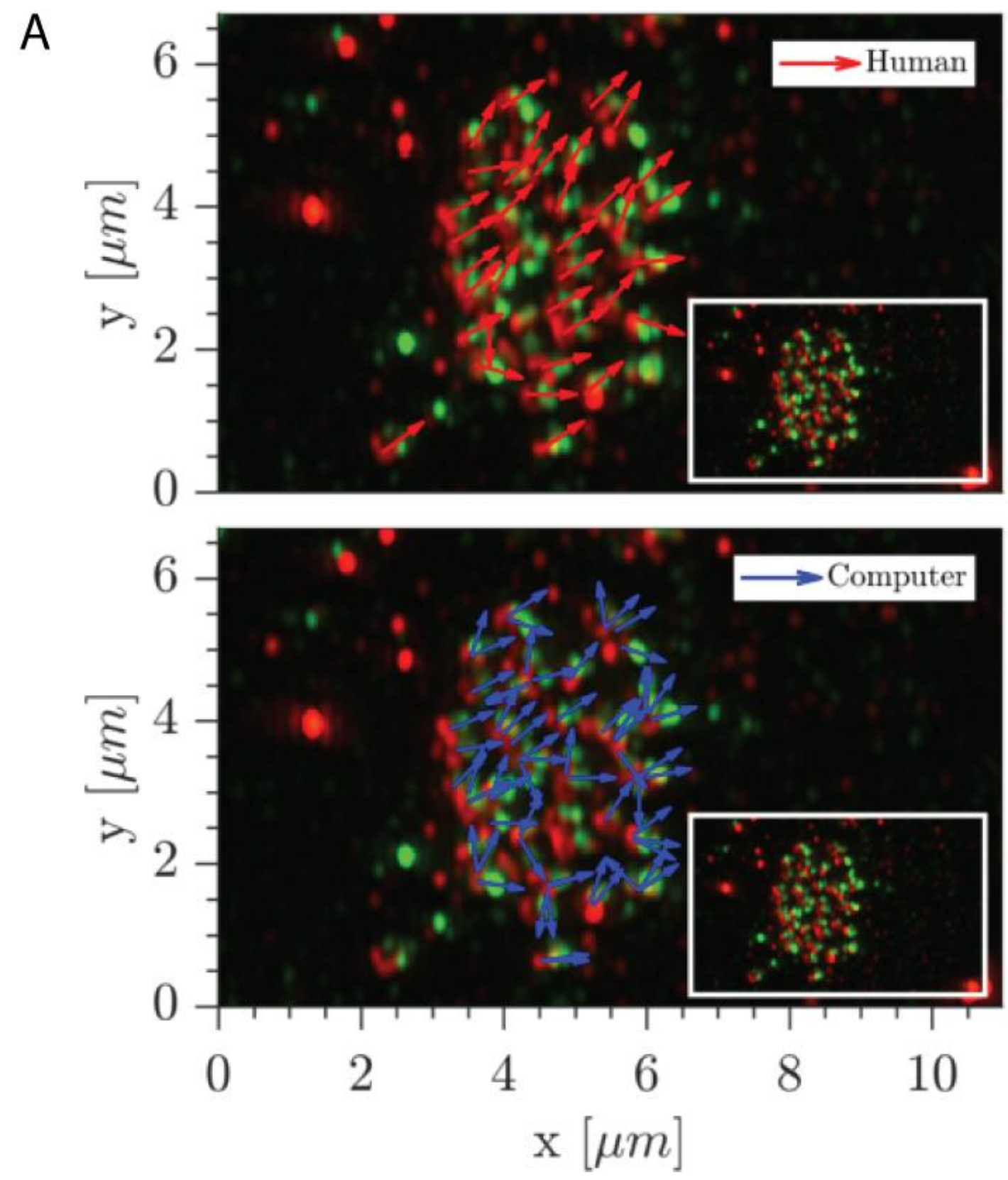




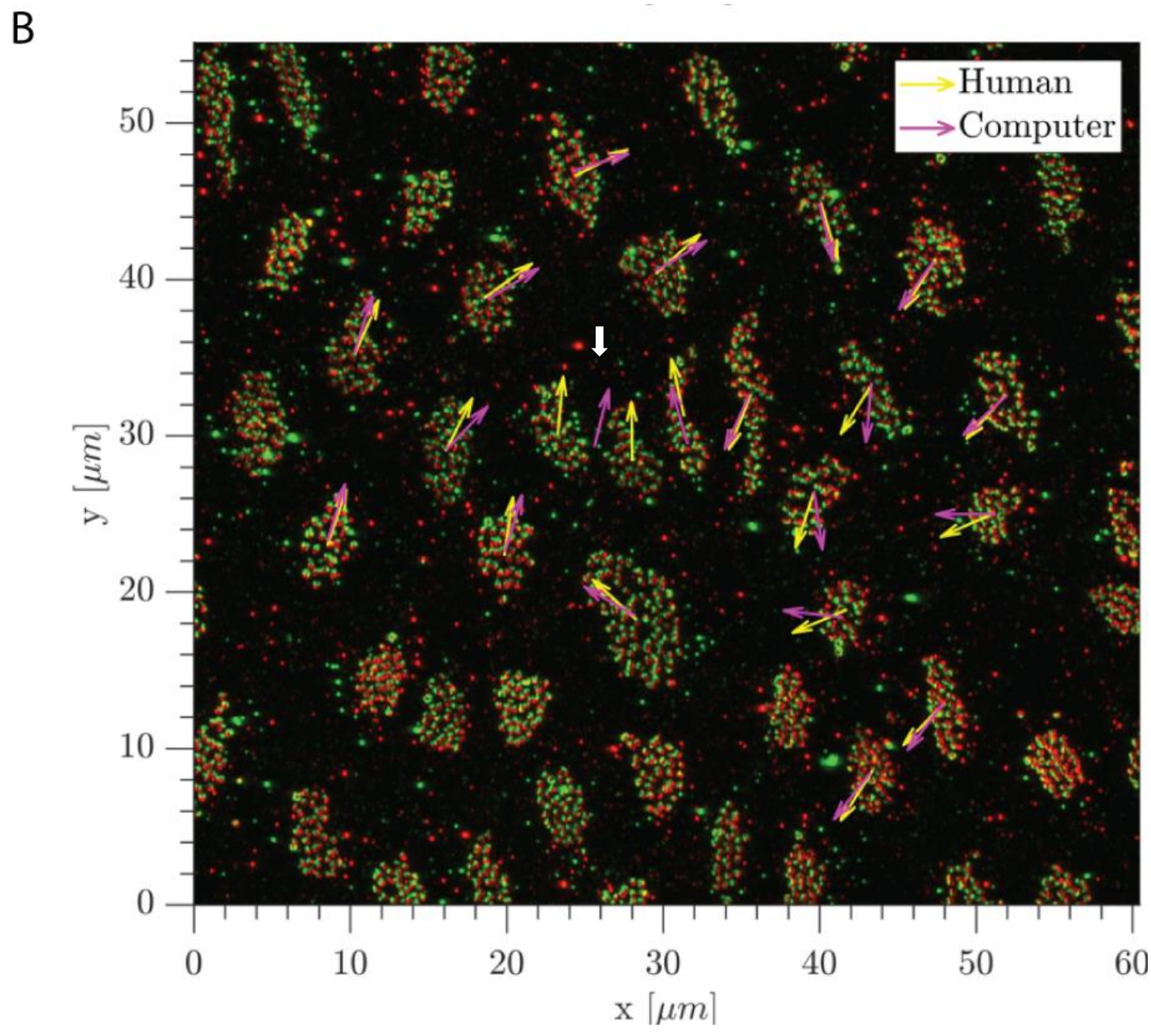

Fig. 18: Comparison between the algorithm and human annotated average rotational polarity direction of a cilia bundle. (A) Each basal body is designated one vector depicting its rotational polarity direction by a human (red), while 2-3 vectors are designated by a computer (blue). (B) The human and computer designated vectors of each basal body - basal foot pair from (A) are averaged, which result in an average rotational polarity direction for each cilia bundle. For two cilia bundles, the computer recognized one large cilia bundle and designated one averaged rotational polarity vector for both cilia bundles, while a human can distinguish 2 separate bundles (white arrow).

In order to investigate how the rotational polarity of cilia correlates with the flow pattern and how it is directed in regions such as the separatrix or in whirls, we first generated a flow-map of the v3V whole mount (Fig.19 A). Next, we stained the same tissue with Odf2 and centriolin. To record the largest area possible, tiles scans of z-stacks were acquired with an overlap of $10 \%$ using a $63 \mathrm{x}$ oil immersion objective. These tile scans were then stitched together and maximum projected. The representative sample in Fig. 19 A (region B), contains a clockwise whirl in the influx region and an anticlockwise whirl ventral from the separatrix (Fig. 19 A, region B and E). The rotational polarities of the cilia bundles of the array of cells that create these whirls also exhibit a clockwise and anti-clockwise pattern, respectively. They differ in their rotational 
polarity direction in a $360^{\circ}$ gradually changing manner to specify those whirls (Fig. $19 \mathrm{~B}, \mathrm{E}$ ). Under straight flows, like that of module one, the cilia bundles of neighboring cells display the same rotational polarity direction and are thus oriented in a unidirectional manner (Fig. $19 \mathrm{~F}$ ). Furthermore, cells generating the opposing flow pattern of modules three and four exhibit rotational polarities that point towards each other, while the cilia bundles of cells at the junction possess rotational polarities facing ventrally (Fig. 19 G).

We quantified the direction of the vectors for module 1, 3, 4, and the separatrix for 3-4 mice. For 2 mice, the direction of vectors in modules 2, and $6 \mathrm{~b}$ was also quantified. These quantifications are visualized in circular plots of these areas (Fig. 20). Rotational polarity in the straight flows of modules $1,2,3,4$, and $6 \mathrm{~b}$ range from $80^{\circ}$ to $170^{\circ}, 170^{\circ}$ to $280^{\circ}, 60^{\circ}$ to $160^{\circ}$, $190^{\circ}$ to $340^{\circ}$, and $100^{\circ}$ to $300^{\circ}$, respectively. In the separatrix, where flows 3 and 4 meet, the rotational polarity directions cover a wide range from $80^{\circ}$ to $300^{\circ}$ (Fig. 20).

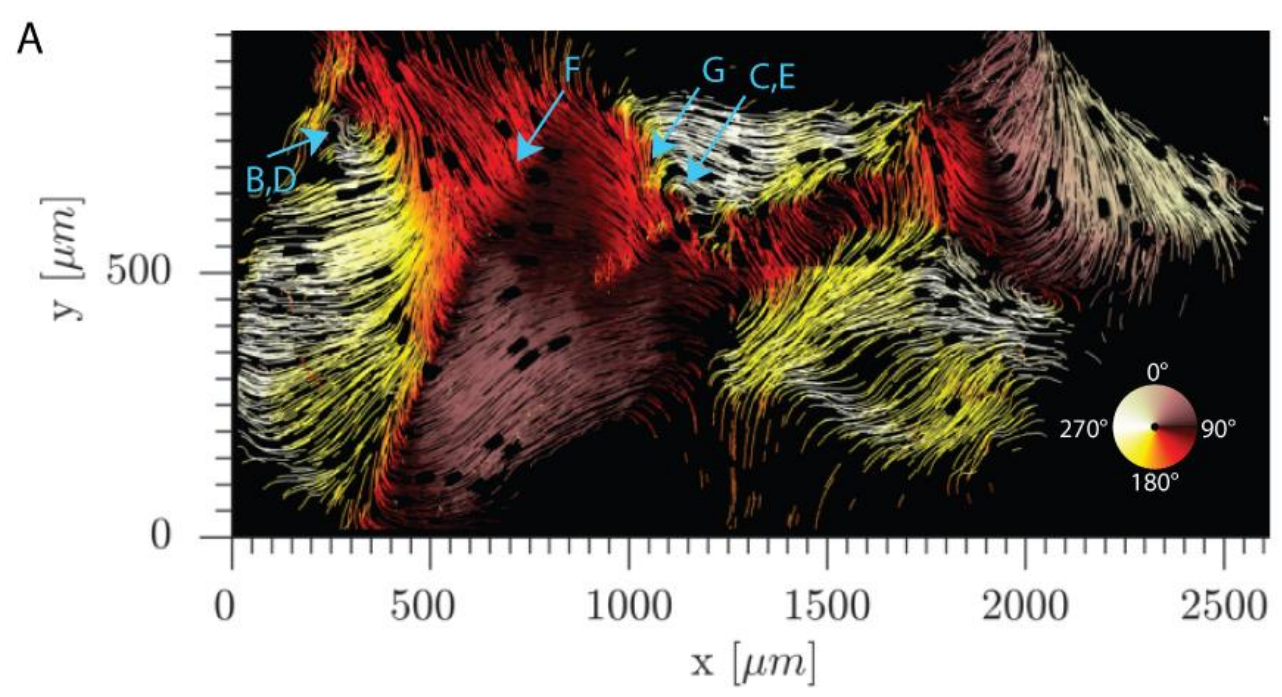

B

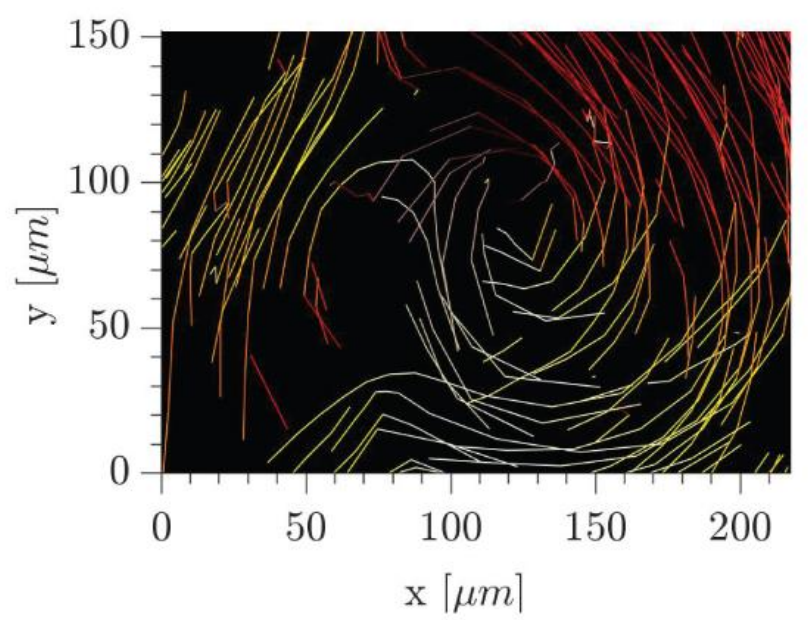

C

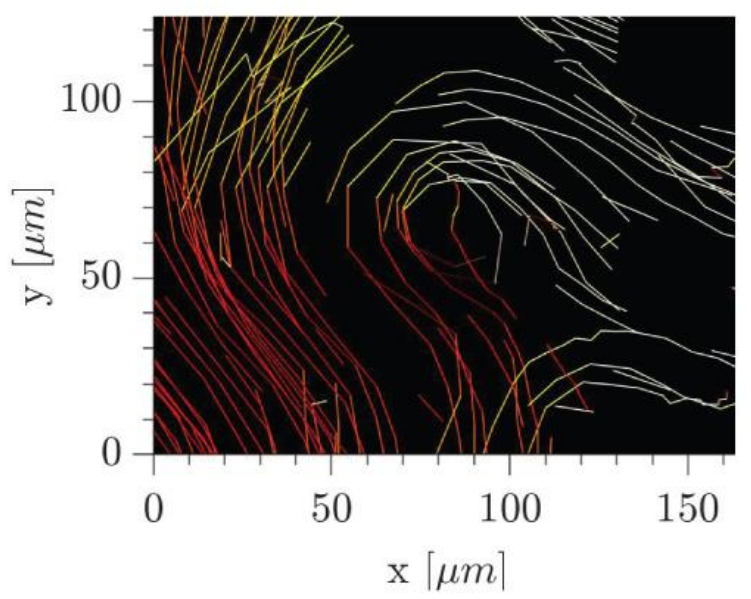


D

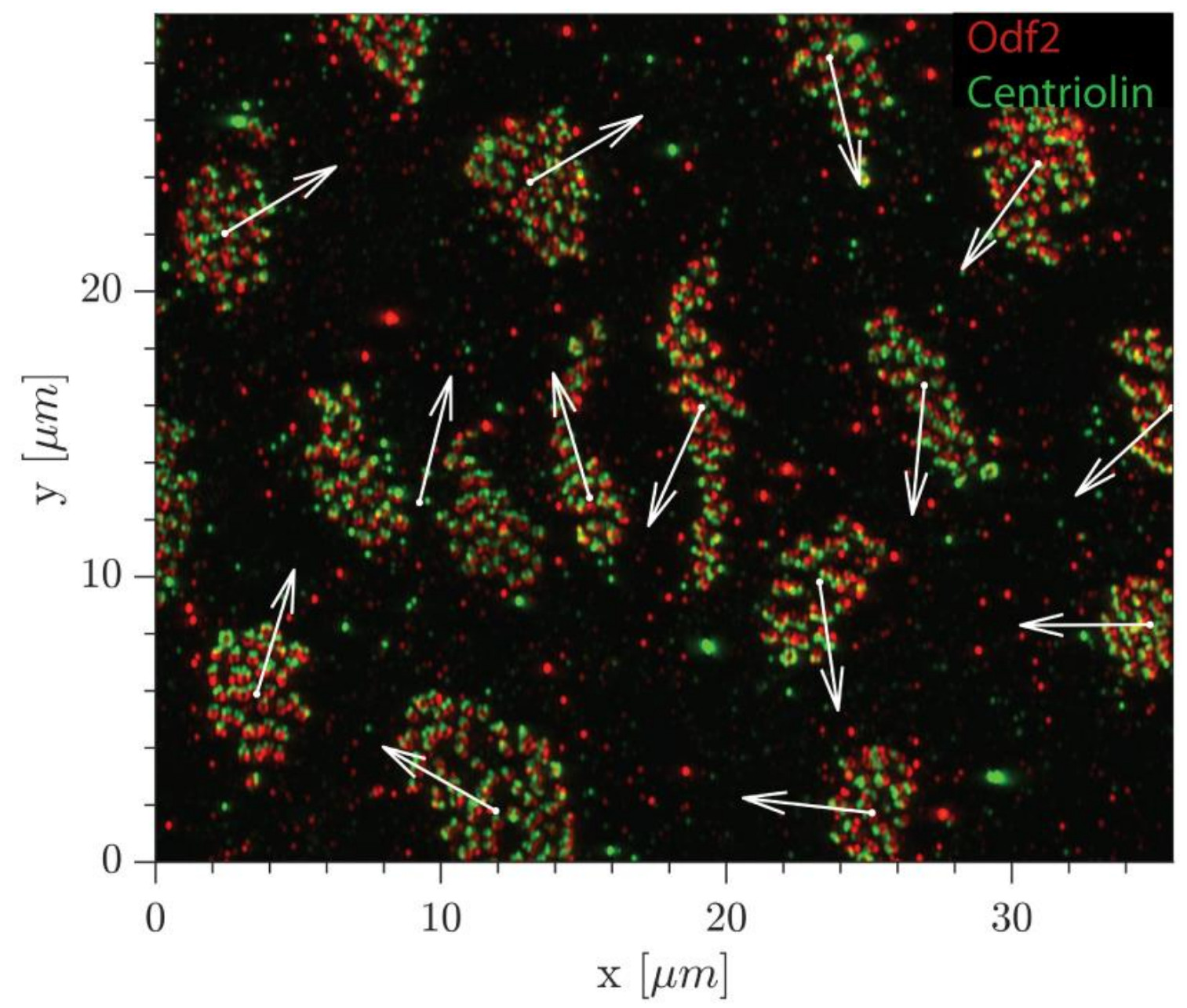

E

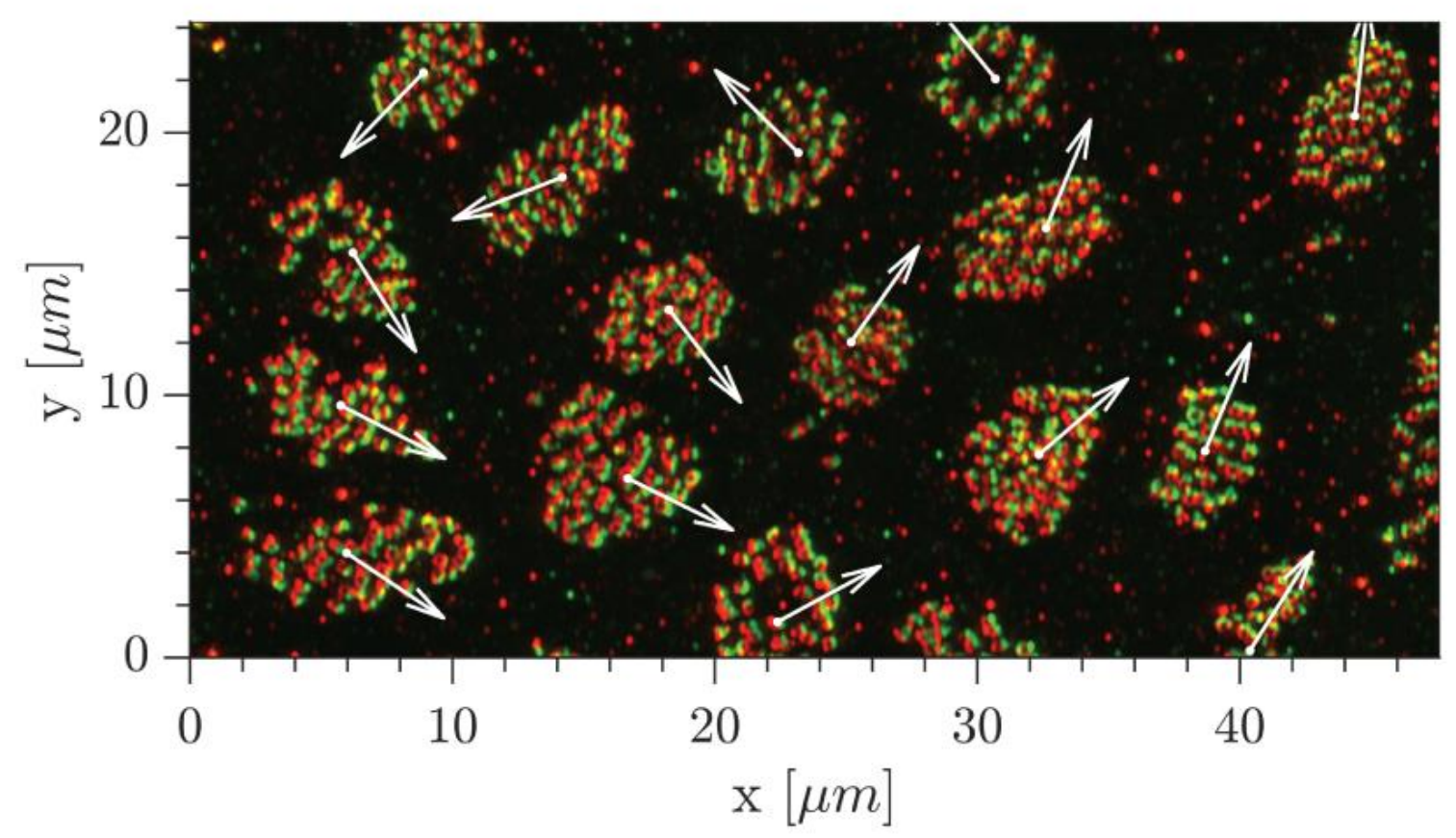


F
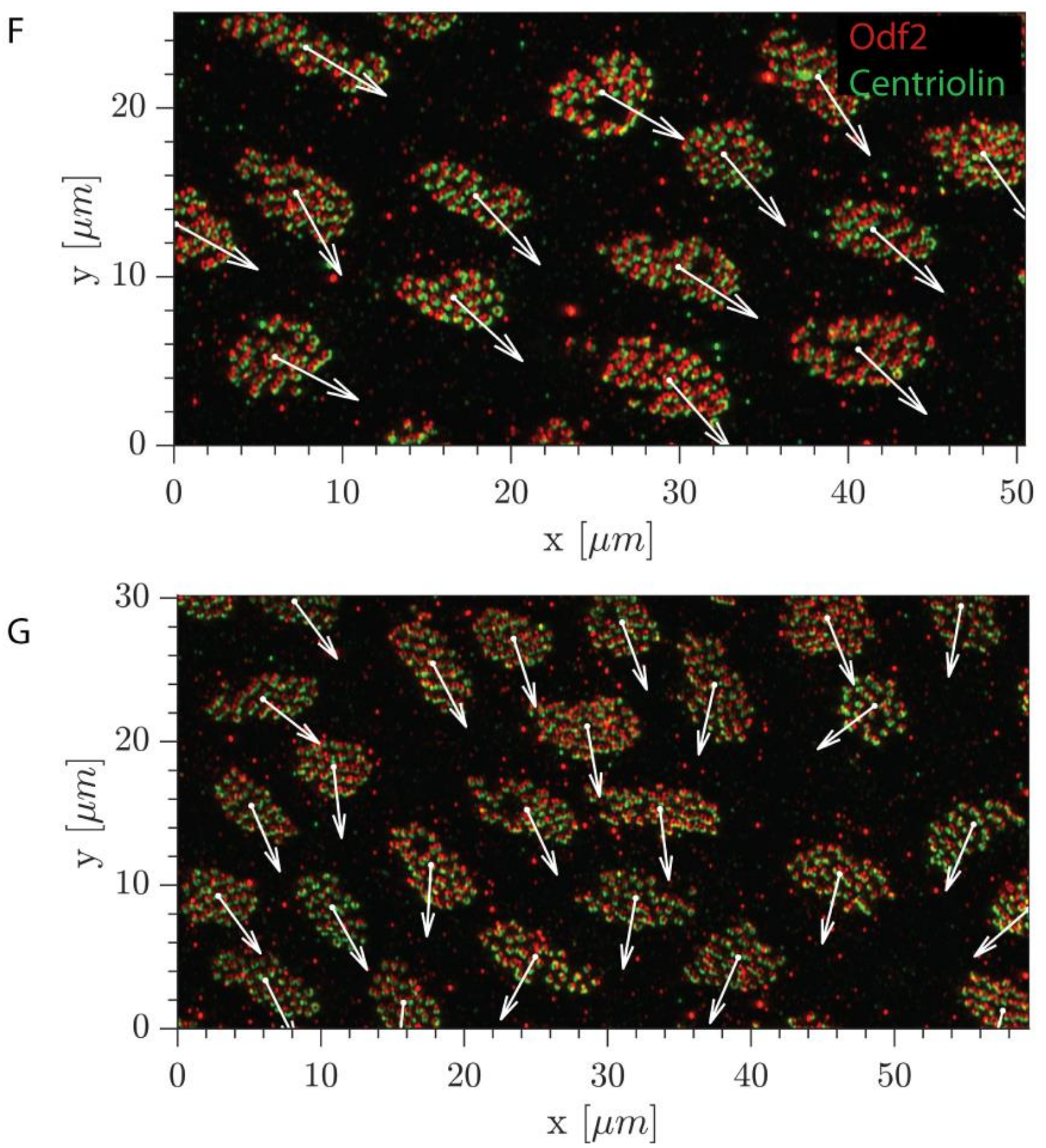

Fig. 19: The rotational polarity presages the flow pattern. (A) V3V flow map of an adult mouse. Blue arrows indicate the area of a clockwise whirl, a straight flow, the separatrix, and a counter-clockwise whirl, for which the rotational polarities are shown in B - D. (B,C) Enlarged images flow map in the area of the clockwise (B) and anticlockwise (C) whirl. (D,E) Immunostaining of Odf2 (red) and centriolin (green) show cells that specify the clockwise and anticlockwise whirl shown in B and C. The rotational polarity of their cilia bundles display a gradually changing directions in a clockwise/anticlockwise manner. (F) The unidirectional flow of module 1 has cells with unidirectional rotational polarities in adjacent cells. (G) The opposing flow pattern of module 3 and 4 is reflected in the rotational polarities of the cells underneath. 

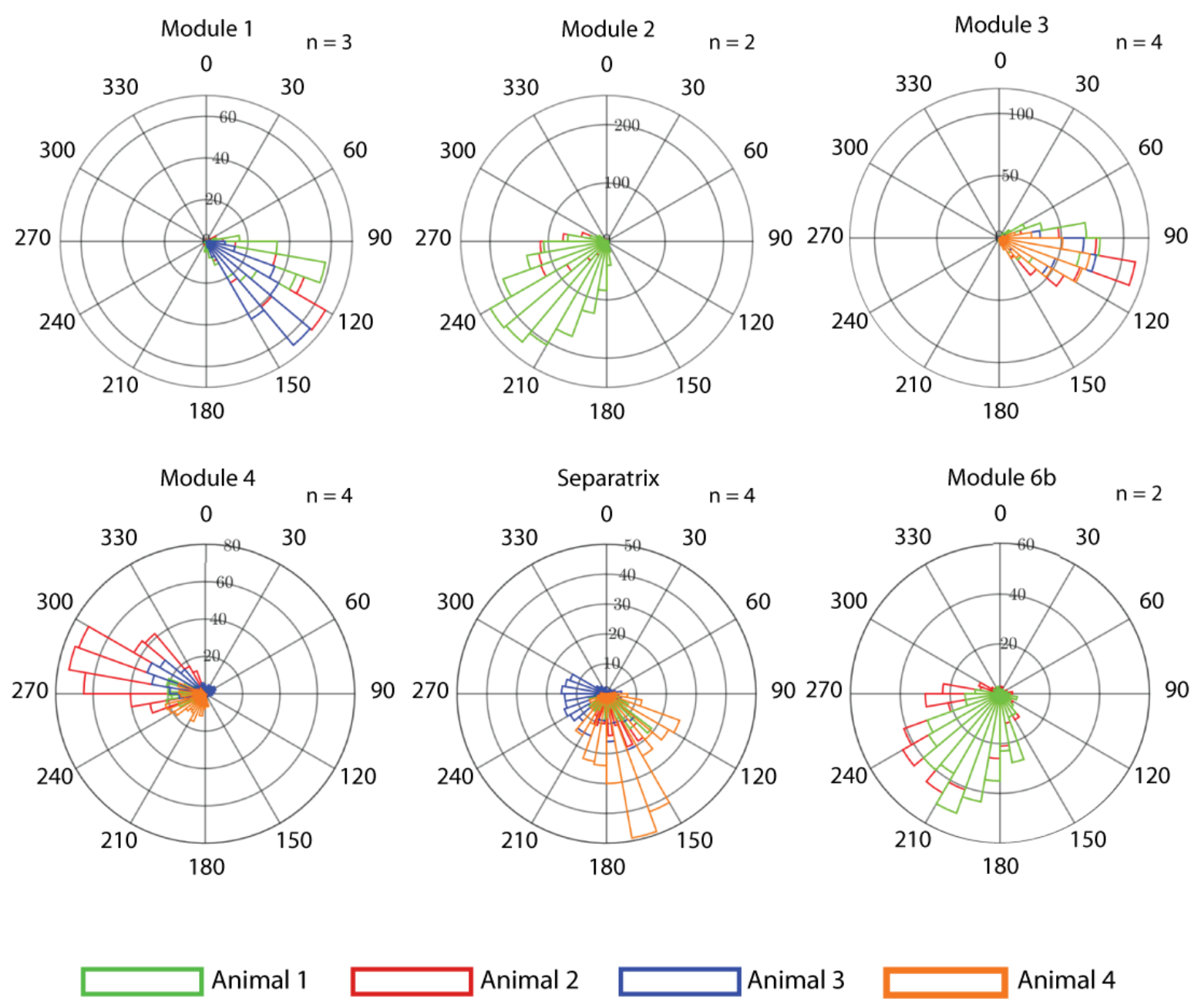

Animal 3

Animal 4

Fig. 20: Circular plots of rotational polarities. For 2 - 4 wild type mice (green, red, blue, and orange bars), circular plots show the quantification of rotational polarity directions of all cells in modules $1,2,3,4,6 \mathrm{~b}$, and the seperatrix. In all mice, directions of rotational polarity of each module is pointed towards similar directions. The given $n$ number for each module refers to the number of mice. Module 1: 753 cilia bundles in 3 animals; Module 2: 2395 cilia bundles in 2 animals; Module 3: 1553 cilia bundles in 4 mice; Module 4: 1212 cilia bundles in 4 mice; Separatrix: 983 cilia bundles in 4 mice; Module 6b: 910 cilia bundles in 2 mice.

These results show that the complex flow pattern of the $\mathrm{v} 3 \mathrm{~V}$ is a result of a complex pattern of translational and rotational polarity directions, which vary depending on the v3V region. The expression of Vangl1 indicates that across the $\mathrm{v} 3 \mathrm{~V}$, PCP proteins are expressed according to the translational and rotational polarity. Furthermore, the rotational polarity is the most accurate to visualize ciliary beating directions. How the cells of the v3V know, in which direction their polarization should occur remains unknown, but it is undoubtedly a phenomenon that occurs during ependymal development. 


\subsubsection{The stereotypical pattern of apical area morphology}

The immunostained $\mathrm{v} 3 \mathrm{~V}$ for $\beta$ catenin and $\gamma$ tubulin was initially used to determine the translational polarity of ependymal cells but additionally, we observed that the apical area sizes, cilia bundle sizes and shapes of cells vary across the tissue and seemed to follow a stereotypical pattern (Fig. 21).

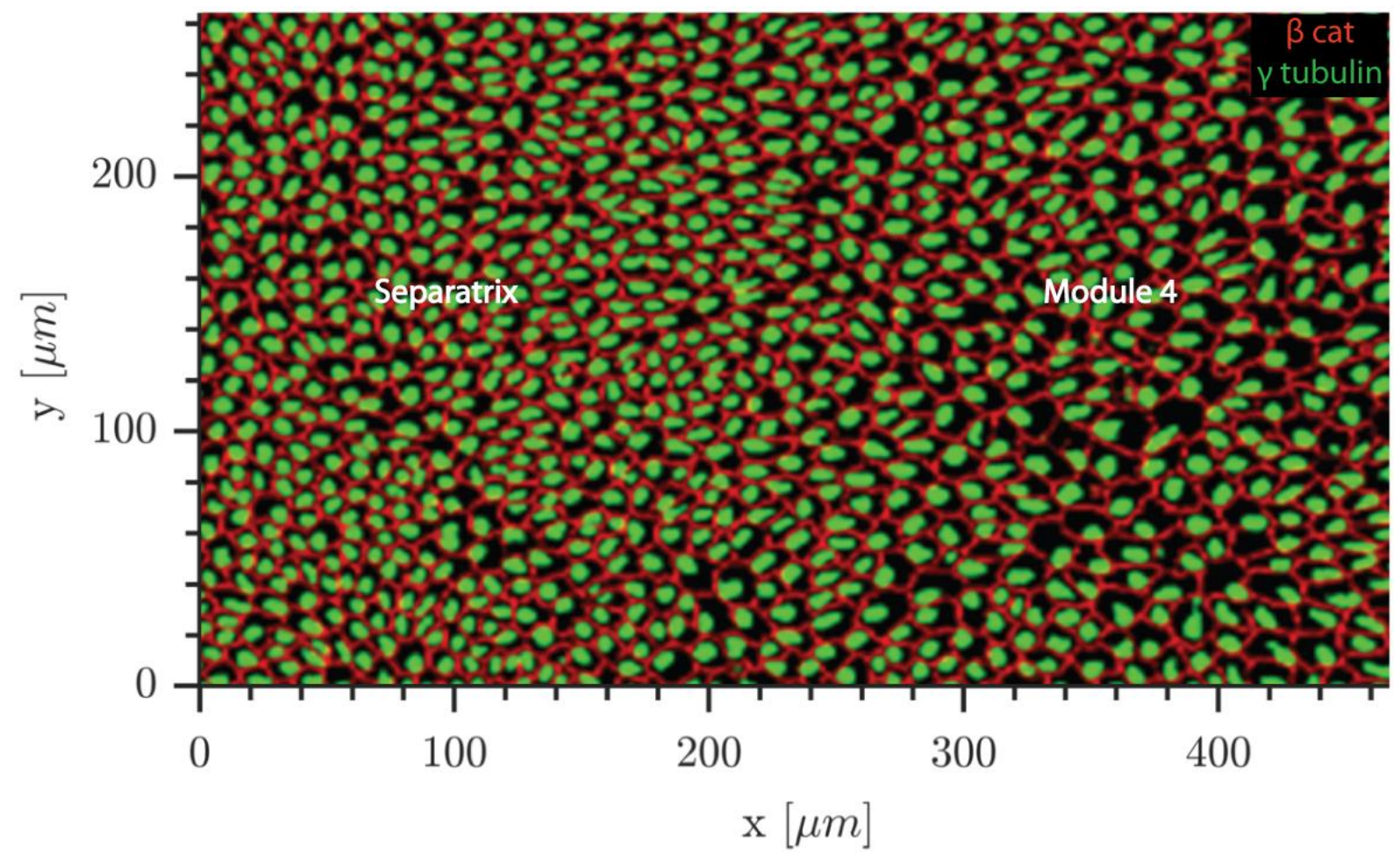

Fig. 21: Maximum intensity projected, stitched, and segmented image, displaying cells of the separatrix and module 4. Note the decreasing cell density from left to right. The Separatrix consists of small and dense cells, whereas flow-module 4 is covered with relatively large cells. 


\subsubsection{Steorotypical pattern of apical area size}

To check, whether the observed variety of apical surface area sizes of the v3V ependyma follows a pattern across multiple mice, we examined the segmented $\beta$ catenin signal and calculated the apical surface area that each polygon surrounds. To quantify apical area sizes for each flow-module in three wild type individuals, we used the same cell boundaries as previously determined to calculate the translational polarity directions for each module (Fig. 22).

The apical area sizes of $\mathrm{v} 3 \mathrm{~V}$ cells range from $2 \mu \mathrm{m}^{2}$ to $250 \mu \mathrm{m}^{2}$. Cells below the size of around $20 \mu \mathrm{m}^{2}$ that are located in the posterior-ventral v3V are mainly tanycytes. Cells with an apical area size above $150 \mu \mathrm{m}^{2}$ (large cells) are clustered in three groups. The first group is located in module one and has three "strands" extending towards the ventral v3V boundary. The first strand is located in the anterior region of module 2, the second strand is located at the junction between module two and eight, while the third strand stretches through module eight. The second group is located in module four, covering a width of $400 \mu \mathrm{m}$ posteriorly, which then stretches down diagonally towards module eight (diagonal band of large cells). The third group is located anterior - dorsally to the tanycyte region. Cells with an apical surface between $80-$ $150 \mu \mathrm{m}^{2}$ are situated in transition areas of cells above $150 \mu \mathrm{m}^{2}$ and below $80 \mu \mathrm{m}^{2}$ (small cells), while the latter is observed in four clusters in the v3V. The first cluster covers module two, while the second cluster is located between the three above described strands of large cells. The third cluster is in module three and eight and is located anterior to the diagonal band of large cells. The fourth cluster is found in module seven. In module $6 \mathrm{~b}$, cells with all apical area sizes are dispersed irregularly. 
A

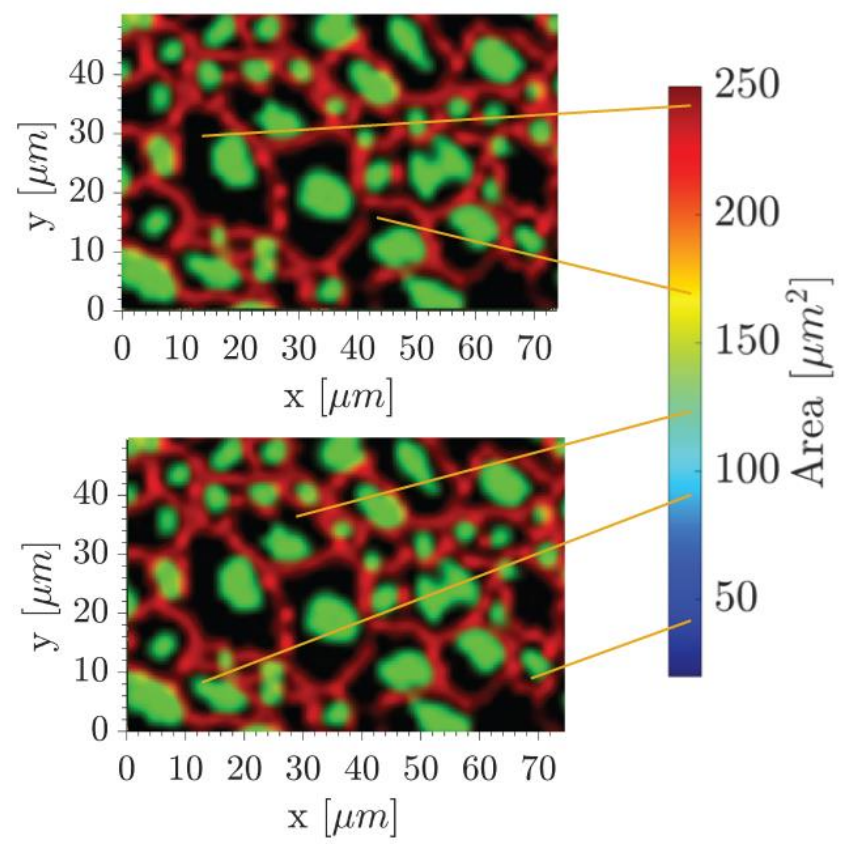

B

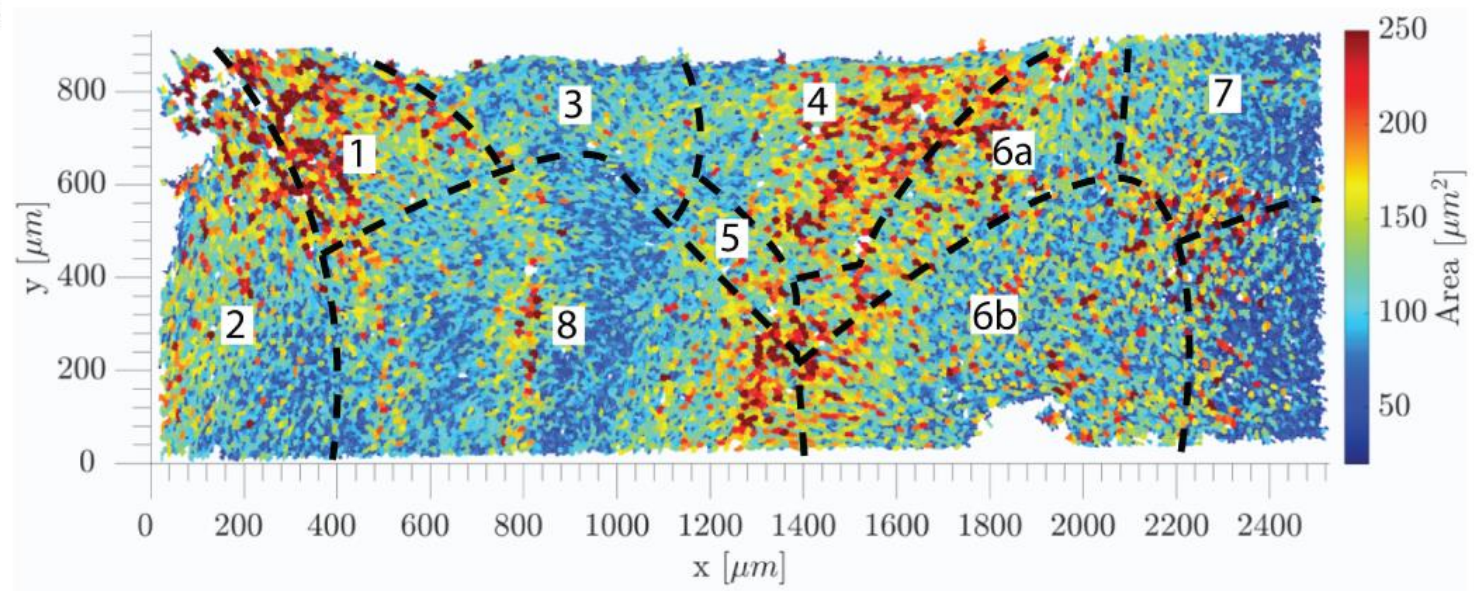

C

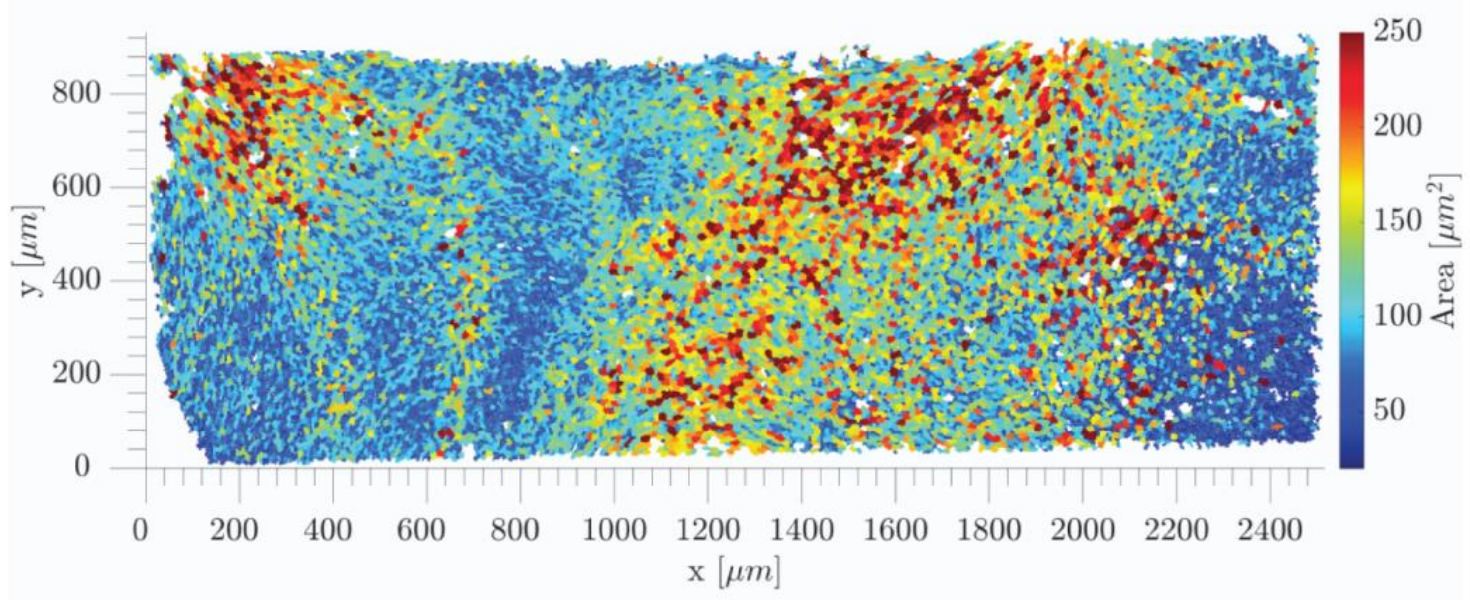




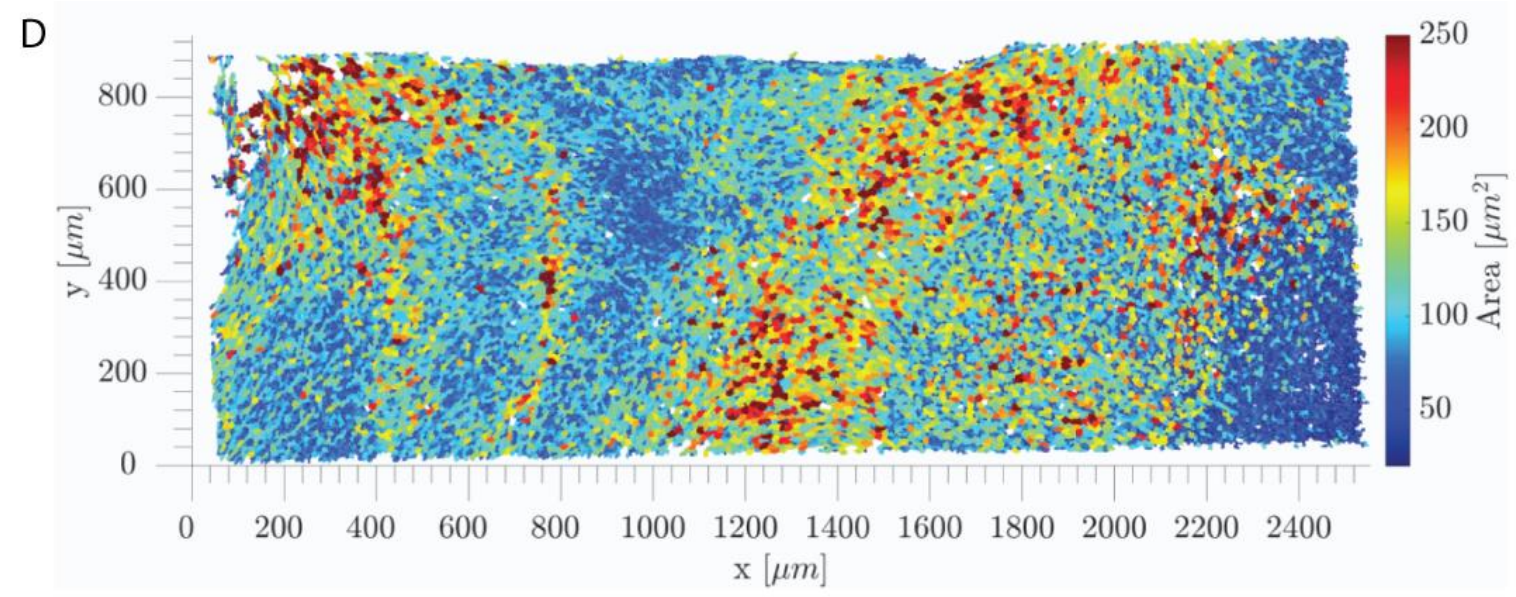

Fig. 22: Stereotypical pattern of cell sizes across the v3V. (A) Example cells with different apical area sizes that are depicted in colors. (B, C, D) Plots of the v3V pattern of cell sizes in 3 wild type mice. Dashed

\subsubsection{A stereotypical pattern of cilia bundle size}

We have shown that apical area sizes in the v3V show a pattern that is very similar in 3 animals. To determine if the size of cilia bundles correlates with the apical area size, we quantified the area of the segmented $\gamma$ tubulin signal and created a map visualizing the size of cilia bundles in v3V ependymal cells.

Reminicent of the pattern of apical area sizes, the ependymal plane is heterogeneous in cilia bundle size, which range from $2 \mu \mathrm{m}^{2}$ to $50 \mu \mathrm{m}^{2}$ (Fig. 23). Cilia bundles above the size of 33 $\mu \mathrm{m}^{2}$ colocalize with the large cells and are also found in three distinct groups. The first group is located in module one and has three strands towards the ventral v3V boundary. The first strand is located in the anterior region of module 2, the second strand is located at the junction between module two and eight, while the third strand stretches through module eight. The second group is located in module four, colocalizing with the above described diagonal band of large cells. The third group is located anterior - dorsally to the tanycyte region. Cells with cilia bundles between $20-33 \mu \mathrm{m}^{2}$ colocalize with cells with an apical area surface between 80 $-150 \mu \mathrm{m}^{2}$ and are found between transition areas of cells with cilia bundles above $33 \mu \mathrm{m}^{2}$ and beneath $20 \mu \mathrm{m}^{2}$, while the latter colocalizes with small cells. These cilia bundles are also clustered in four groups. The first cluster covers module two, while the second cluster is located between the above described strands of cells, that possess cilia bundles above the size of 33 $\mu \mathrm{m}^{2}$. The third cluster is in module three and eight and is located anterior to the second group of cells that possess cilia bundles above the size of $33 \mu \mathrm{m}^{2}$. The fourth cluster is located in 
module 7. Cilia bundles under the size of $4 \mu \mathrm{m}^{2}$ likely reflect the cilia of tanycytes, which only possess 1-2 cilia. In module $6 \mathrm{~b}$, cells with all cilia bundle sizes are dispersed irregularly.

We show that the pattern of cilia bundle size correlates with the pattern of apical area sizes. This indicates that the cilia bundle size may depend on the apical area size and thus the size of the cells.

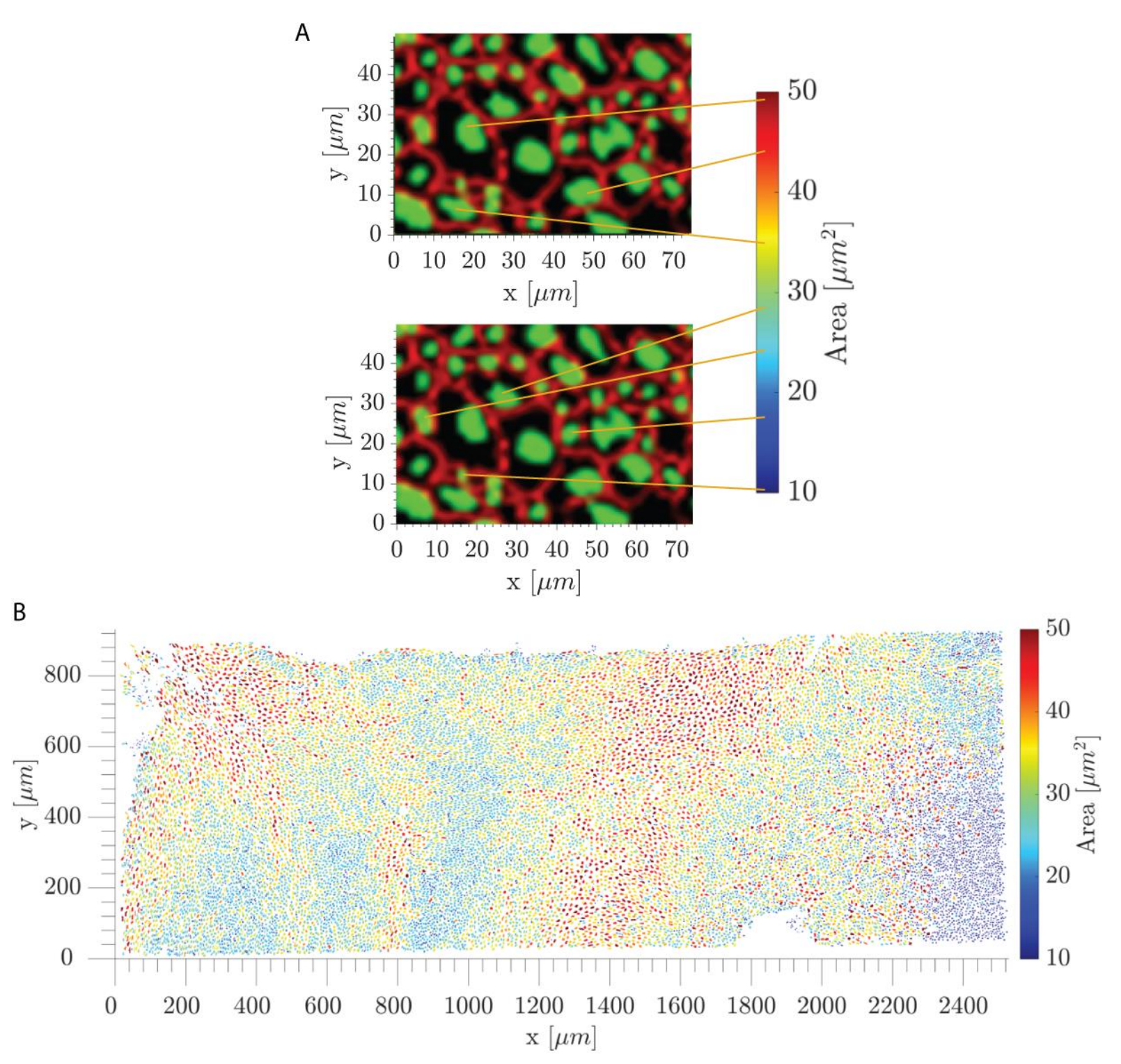




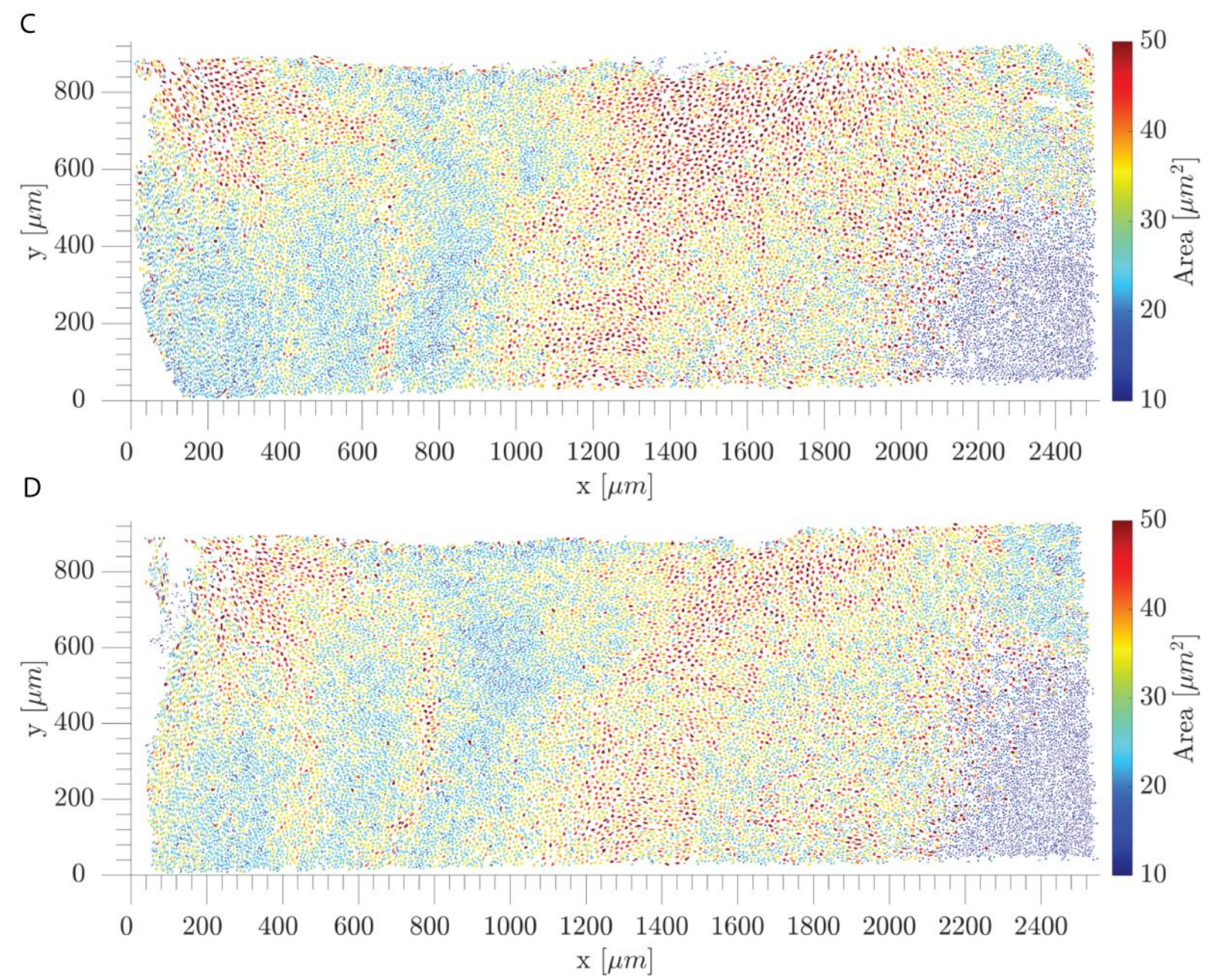

Fig. 23: Stereotypical pattern of the size of cilia bundles. (A) Example cells with different cilia bundle sizes that are depicted in colors. (B,C,D) Plots of the v3V pattern of cilia bundle sizes in 3 wild type animals.

\subsubsection{Distance between centroids}

While the direction of the $\overrightarrow{C C}$ vector determines the translational polarity direction, its length can be measured to show the degree of translational polarization.

The length of the $\overrightarrow{C C}$ vectors mostly correlates with the apical area size and cilia bundle size and shows a stereotypical pattern in three mice (Fig. 24). $\overrightarrow{C C}$ vectors above a length of $3 \mu \mathrm{m}$, colocalize with large cells and cilia bundles above the size of $33 \mu \mathrm{m}^{2}$ and are thus also found clustered in three groups. The first group is located in module one with three strands. The first strand is located in the anterior part of module 2, the second strand is situated at the junction between module two and eight, while the third strand stretches through module eight. The 
second group is located in module four, covering a width of $400 \mu \mathrm{m}$ posteriorly, which then stretches down diagonally towards module eight. The third group is located anterior - dorsally to the tanycyte region. $\overrightarrow{C C}$ vectors between 2 - $3 \mu \mathrm{m}$ colocalize with cells with an apical area surface between $80-150 \mu \mathrm{m}^{2}$ and cilia bundles between $20-33 \mu \mathrm{m}^{2}$. These $\overrightarrow{C C}$ vectors are found between transition areas of $\overrightarrow{C C}$ vectors above $3 \mu \mathrm{m}$ and beneath $2 \mu \mathrm{m}$, while the latter four groups. The first group covers the anterior part of module eight. The second group is found in module three, stretching ventrally to cover the posterior area of module 8 . The third group is located in module $6 \mathrm{~b}$ and the fourth in module 7 . In module $6 \mathrm{~b}, \overrightarrow{C C}$ vectors of above the length of $3 \mu \mathrm{m}$ are also found but are not distributed as abundantly as cells with large apical areas or cilia bundles (Fig 24).

A long $\overrightarrow{C C}$ vector indicates that a relatively large area of the apical surface lacks motile cilia. It has been suggested that the cilia free area in ependymal cells could be involved in releasing chemokines such as noggin, which promotes adult neurogenesis in the V-SVZ $(26,27)$. This area is most likely covered with structures that have a physiological function. Therefore, the control over the degree of translational polarization of a cell may contribute to the cells function.

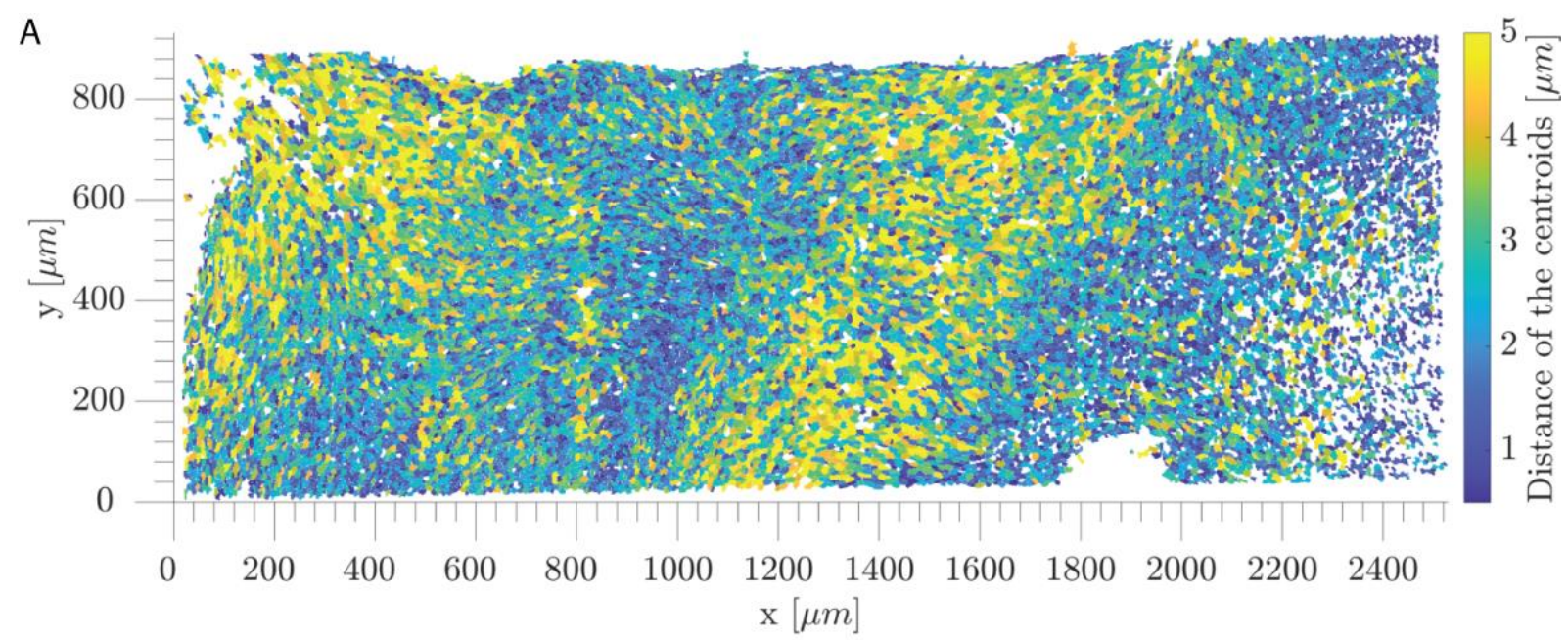



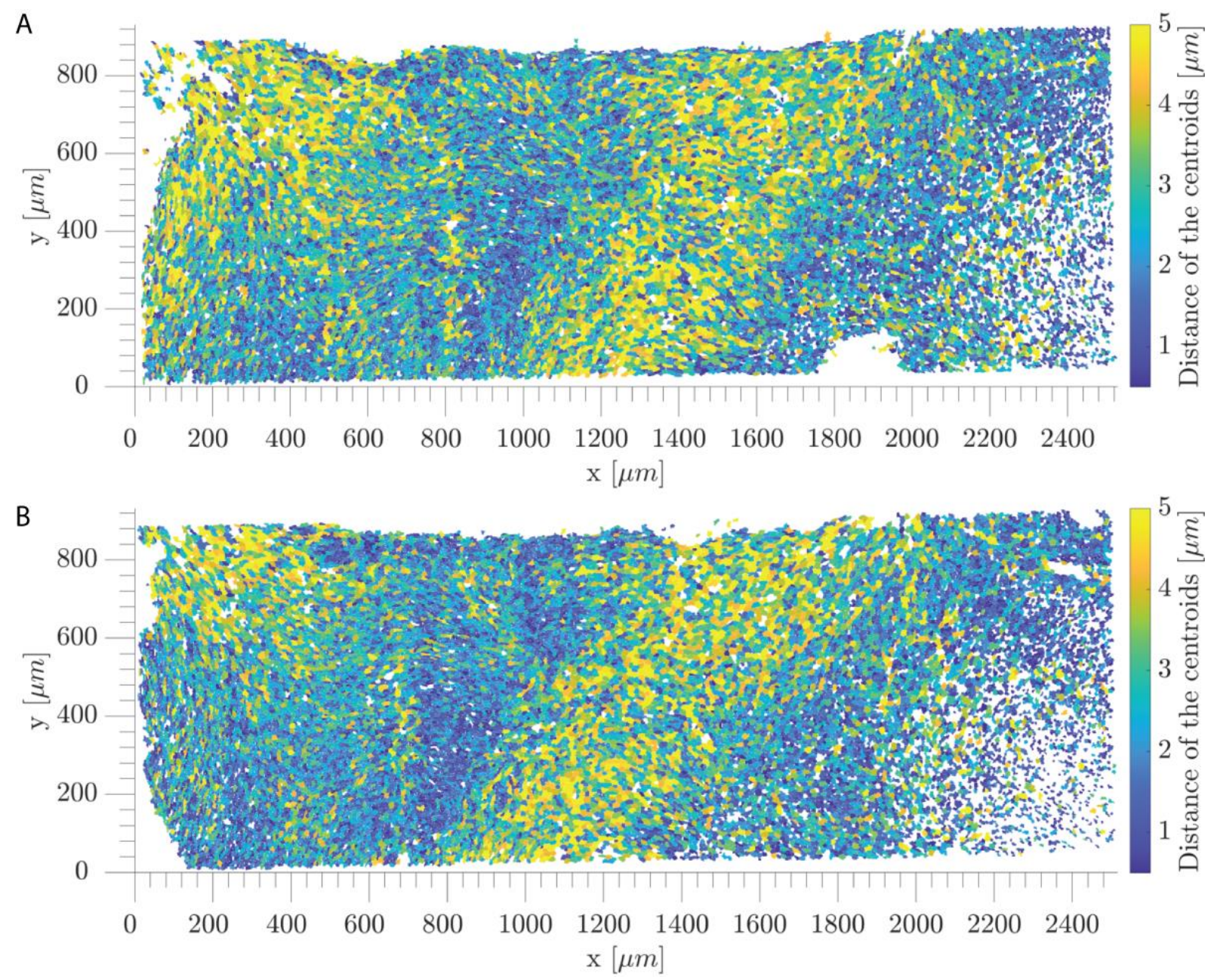

Fig. 24: Stereotypical pattern of the $\overrightarrow{\mathbf{C C}}$ vector length distribution. (A,B,C) Plots of the v3V pattern of $\overrightarrow{\mathbf{C C}}$ vector lengths in 3 wild type mice.

\subsubsection{The eccentricity pattern of cilia bundles}

To investigate the pattern of cilia bundle shapes, we checked the segmented $\gamma$ tubulin stainings for the eccentricity of the cilia bundles. 0 represents a round cell and the value 1 represents a line-shaped bundle. We observe that the v3V ependyma displays cilia bundles with various eccentricities that follow a particular pattern as well (Fig. 25).

The above described small cells that have small cilia bundles, seem to possess cilia bundles that are highly eccentric (>0.8), except for some in module 8. Cilia bundles of this shape are also found in the most anterior area, where they stretch throughout the y-axis and cover up to 400 $\mu \mathrm{m}$ of the $\mathrm{x}$-axis, covering module 1 and parts of module 2 . In the above described diagonal strand of large cells and cilia bundles above the size of $33 \mu \mathrm{m}^{2}$, there are two groups of highly eccentric cilia bundles. One is located dorsally, beginning from the anterior edge of module 4 
and span up to $200 \mu \mathrm{m}$ on the $\mathrm{x}$-axis and around $300 \mu \mathrm{m}$ on the $\mathrm{y}$-axis. The second group of highly eccentric cilia bundles is positioned ventrally at the v3V center and stretch diagonally in the dorsal direction up to $400 \mu \mathrm{m}$ on the y-axis. The area of module $6 \mathrm{~b}$, where apical area and cilia bundle sizes are dispersed, also the cilia bundle shapes are dispersed. Moderately eccentric cilia bundles $(0.65-0.8)$ surround the highly eccentric bundles and those with an eccentricity lower than 0.65 , cover the rest of the $\mathrm{v} 3 \mathrm{~V}$. All cilia bundles have an eccentricity value of at least 0.5 (Fig. 25).

Interestingly, many areas with relatively small apical area seem to have an increased number of highly eccentric bundles, which may be a mechanism to ensure bundle displacement and thus a cilia free area of the apical surface in those cells.

A

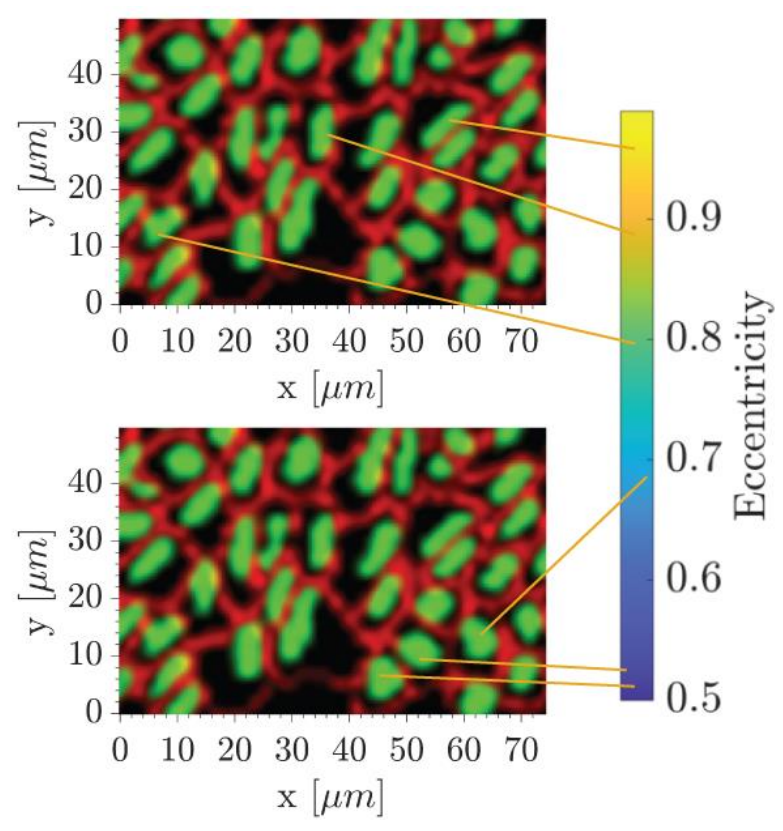

B

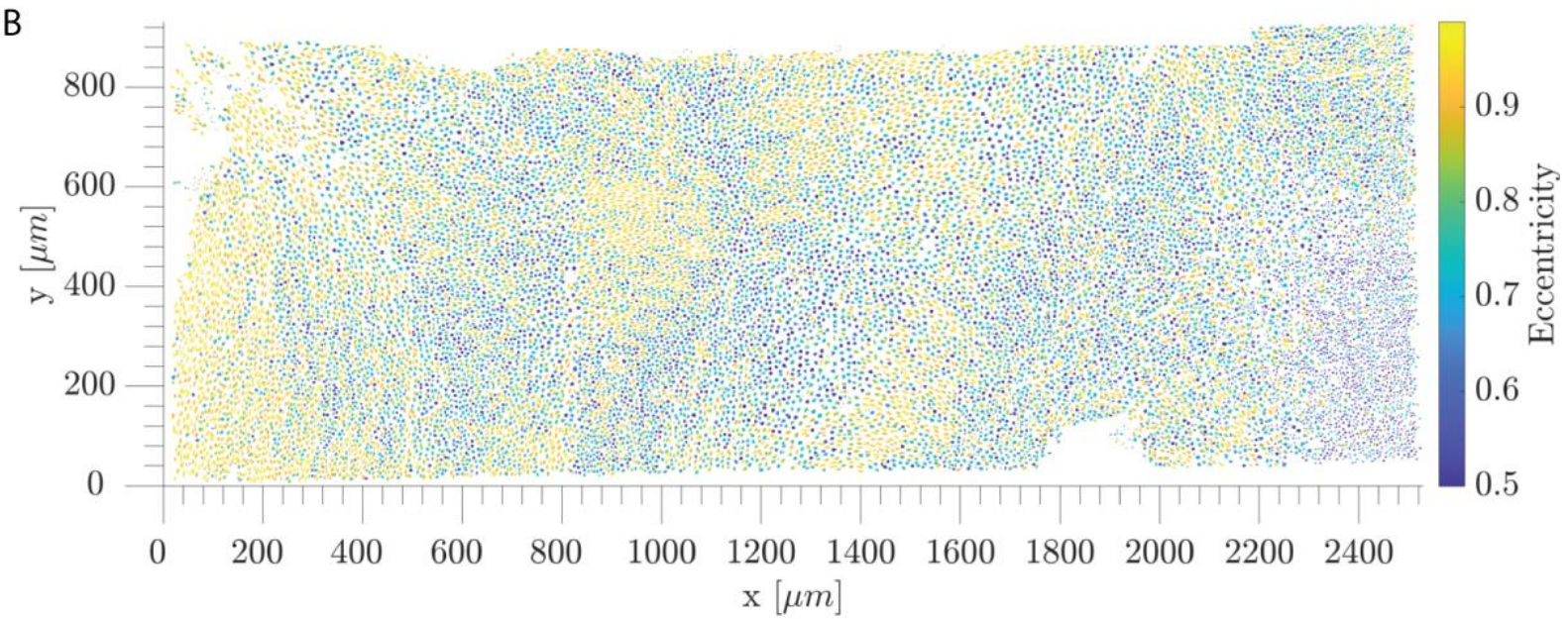



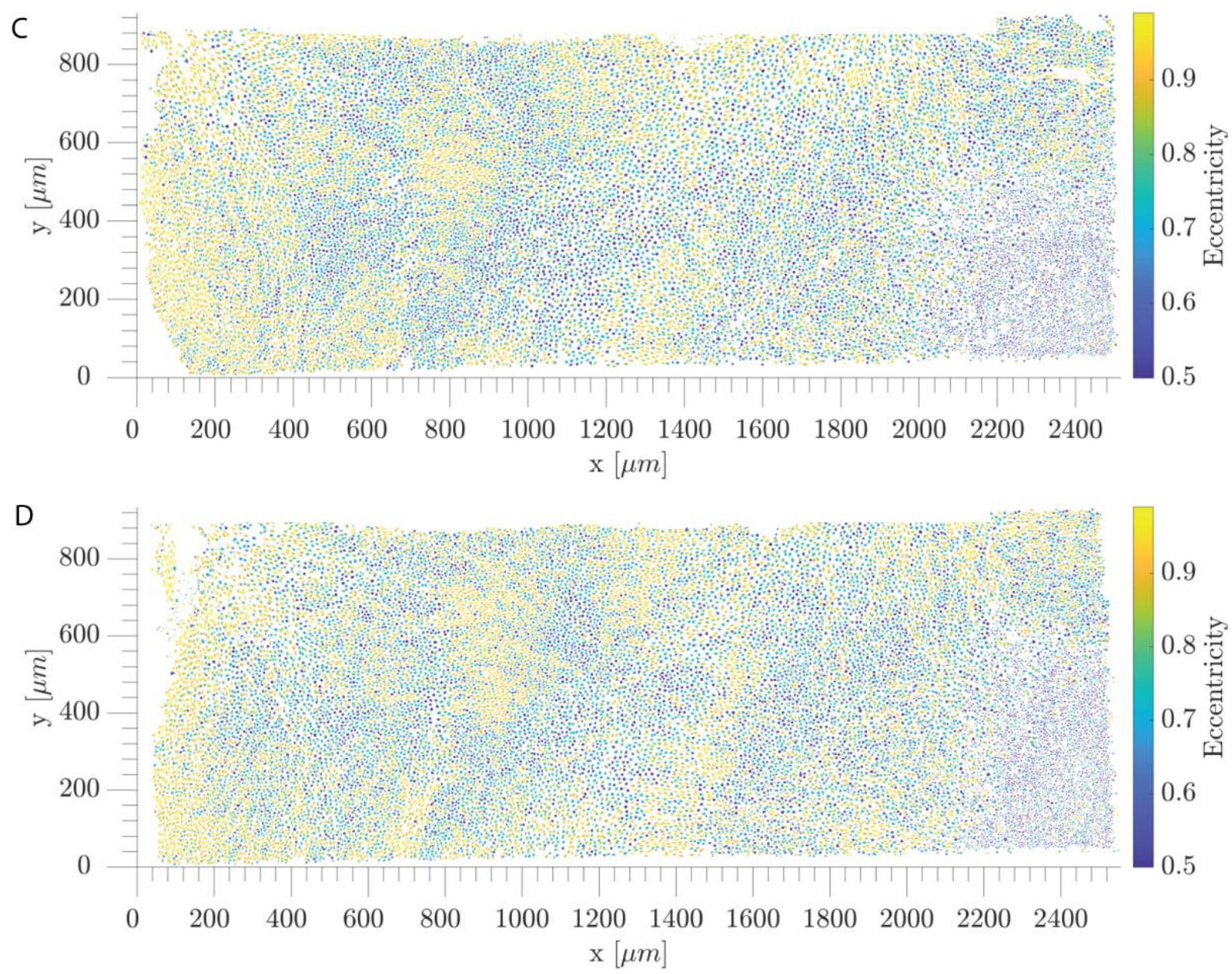

Fig. 25: Stereotypical pattern of the eccentricity of cilia bundles. . (A) Example cellswith different eccentricities that are depicted in colors. (B,C,D) Plots of the v3V pattern of cilia bundle eccentricity of 3 wild type mice.

Altogether, we conclude that the $\mathrm{v} 3 \mathrm{~V}$ architecture is predetermined to form a particular pattern of apical area sizes, cilia bundle sizes and shapes, and degree of cilia bundle displacement. This displays a hard-wired control of ependymal plane formation, which probably occurs due to cellintrinsic mechanisms in response to environmental cues during ependymal development. Whether the cellular architecture is related to the polarization of ependymocytes remains to be investigated. 


\subsection{Age-dependent changes in the $\mathrm{v} 3 \mathrm{~V}$ flow pattern}

The position of cilia bundles, translational and rotational polarities are established by P21 (20). Based on studies on the lateral ventricle it is thought that after P21, these parameters remain constant implying that there will not be a change in the flow pattern after that time. We determined flow maps of $\mathrm{v} 3 \mathrm{~V}$ whole mounts of mice at the age of postnatal day P20 - 24, P70 - P84, 6 months, 9 months and 1.5 years. We found that the flow pattern of young mice was notably different from that of adult mice and discovered that in the $\mathrm{v} 3 \mathrm{~V}$, there are significant and systematic changes in the flow pattern.

\subsubsection{Age-dependent whirl formation}

We compared $\mathrm{v} 3 \mathrm{~V}$ flow maps of mice at the above mentioned ages and quantified the occurrence of whirls that are located ventral from the separatrix (central whirl). As shown in table 4, only $7 \%$ of the young mice (P20 - P24) show a whirl. By P70 - P84, this fraction is close to $50 \%$ and by month 8 and older, all individuals have a central whirl. These whirls at this location are always anticlockwise. Occasionally, mice older than 6 months exhibit an additional whirl situated between modules 6 and 8, which rotates clockwise (Fig. 26). Some mice also exhibited clockwise whirls in module 2 (Fig. 19 A).

Table 4 Quantification of the occurrence of a central whirl.

\begin{tabular}{|l|l|l|l|}
\hline Age & $\begin{array}{l}\text { Number of mice with } \\
\text { a central whirl }\end{array}$ & Number of mice (n) & $\begin{array}{l}\text { Percentage of mice } \\
\text { with a central whirl }\end{array}$ \\
\hline P20 - P24 & 1 & 13 & 7 \\
\hline P70 - P84 & 20 & 46 & 43 \\
\hline 6 months & 9 & 9 & 93 \\
\hline 9 months & 9 & 9 & 100 \\
\hline 1.5 years & 7 & 7 & 100 \\
\hline
\end{tabular}




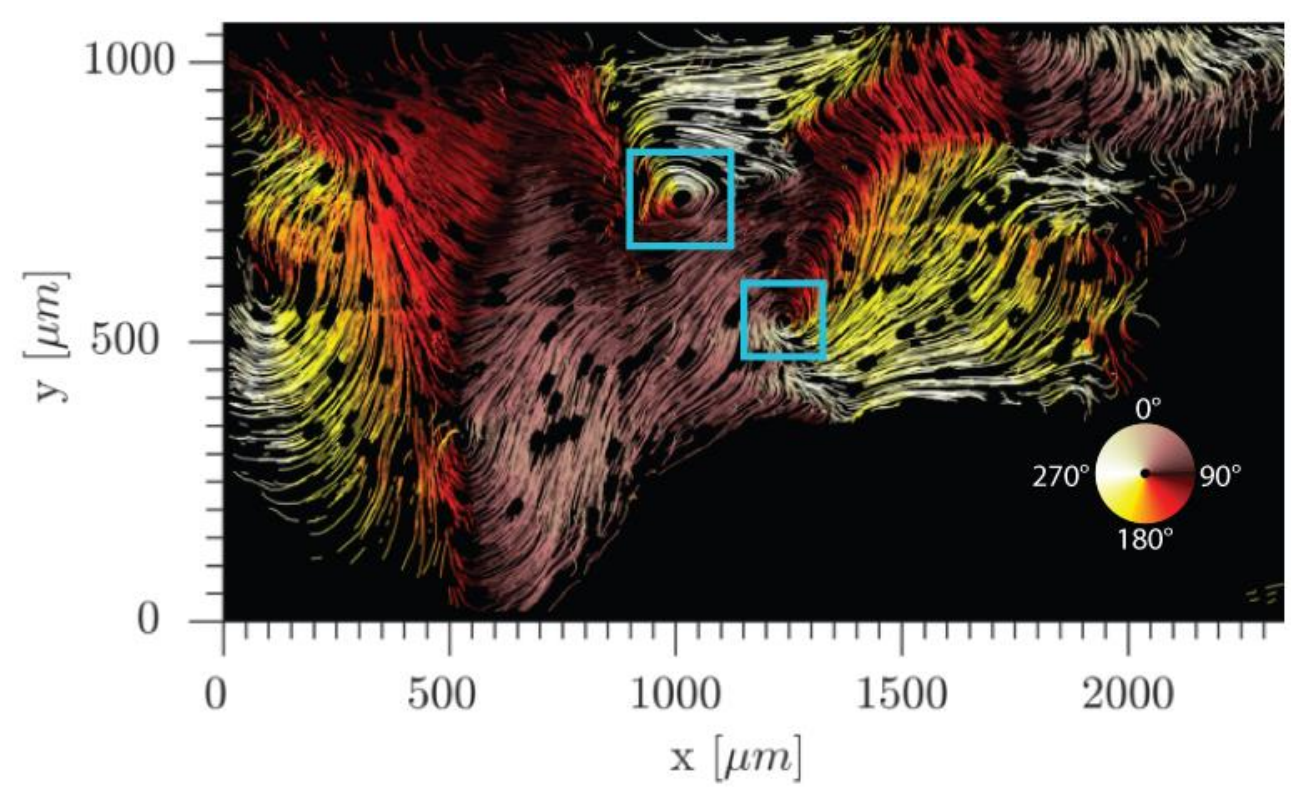

Fig. 26: Flow-map of a v3V, isolated from a 6 months old mouse. Blue squares indicate an anti-clockwise central whirl dorsally and an additional clock-wise whirl between module 6 and 8 .

Here we show that the whirl like pattern in the flow is a process, which immerges over time and depending on the localization in the $\mathrm{v} 3 \mathrm{~V}$ its orientation can be clockwise or anti-clockwise. Interestingly, all observed whirls occur either where flows of different directions come together or display bending, like the flow of module 2. It is possible that those areas of the v3V flow pattern that show a bend in their flow, over time form a whirl due to hydrodynamic forces the bend of flow creates. Thus depending on the localization of the whirl its orientation (clockwise or anticlockwise) can be predicted.

\subsubsection{The ventral $\mathrm{v} 3 \mathrm{~V}$ is dynamic}

Further interesting observations were made by comparing the flow pattern of mice at the age of P20 - P24 and P70 - P84. While modules 5 and 8 changed their area size, the flow pattern of module $6 \mathrm{~b}$ changed its direction from towards the tanycytes to away from them (Fig. 27, 28). We first ought to compare the sizes of modules in young and older mice. We used a custom made graphical interface (GUI), written by Dr. Christian Westendorf, to determine each module according to flow directions. Using this GUI, we manually marked each module in the flow maps. This way the area size of each module was computed. Taking the mean of four mice at P20 - P24, module five has an area of $1.49 \times 10^{5}$ and module 8 an area of $1.79 \times 10^{5}$. At P70 - 
P84 the mean of three mice shows how the area of module 5 decreased to $0.27 \times 10^{5}$, whereas the area of module 8 increased to $3.54 \times 10^{5}$ (Fig.27).

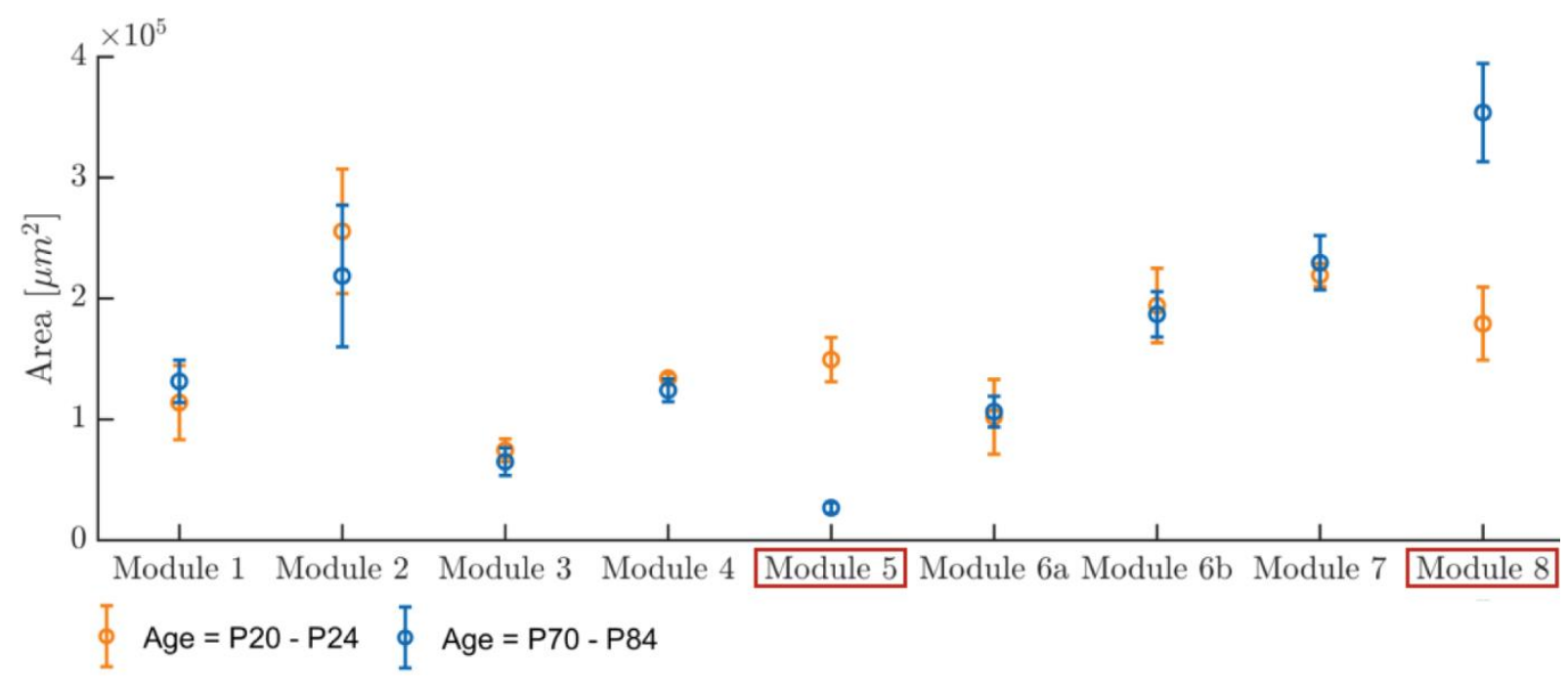

Fig. 27: A comparison between the area size of each module in young (P20 - P24) and adult (P70 - P84) mice. With the increase in age, module 5 decreases and module 8 increases in size. $(\mathrm{n}(\mathrm{P} 20-\mathrm{P} 24)=4 ; \mathrm{n}(\mathrm{P} 70-\mathrm{P} 84)=$ $3)$.

The observed changes in flow direction upon maturation are restricted to the ventral part of the v3V. The dorsal flows 1, 3, 4, and 7 do not change between young and adult individuals (Fig. 28). This indicates the presence of a dorsoventral boundary separating a dorsal, static region, and a ventral, dynamic compartment. It should be recalled, that the separatrix and the whirl underneath the separatrix establish an anteroposterior compartment boundary (18). Together these two boundaries would subdivide the $\mathrm{v} 3 \mathrm{~V}$ into four compartments. 

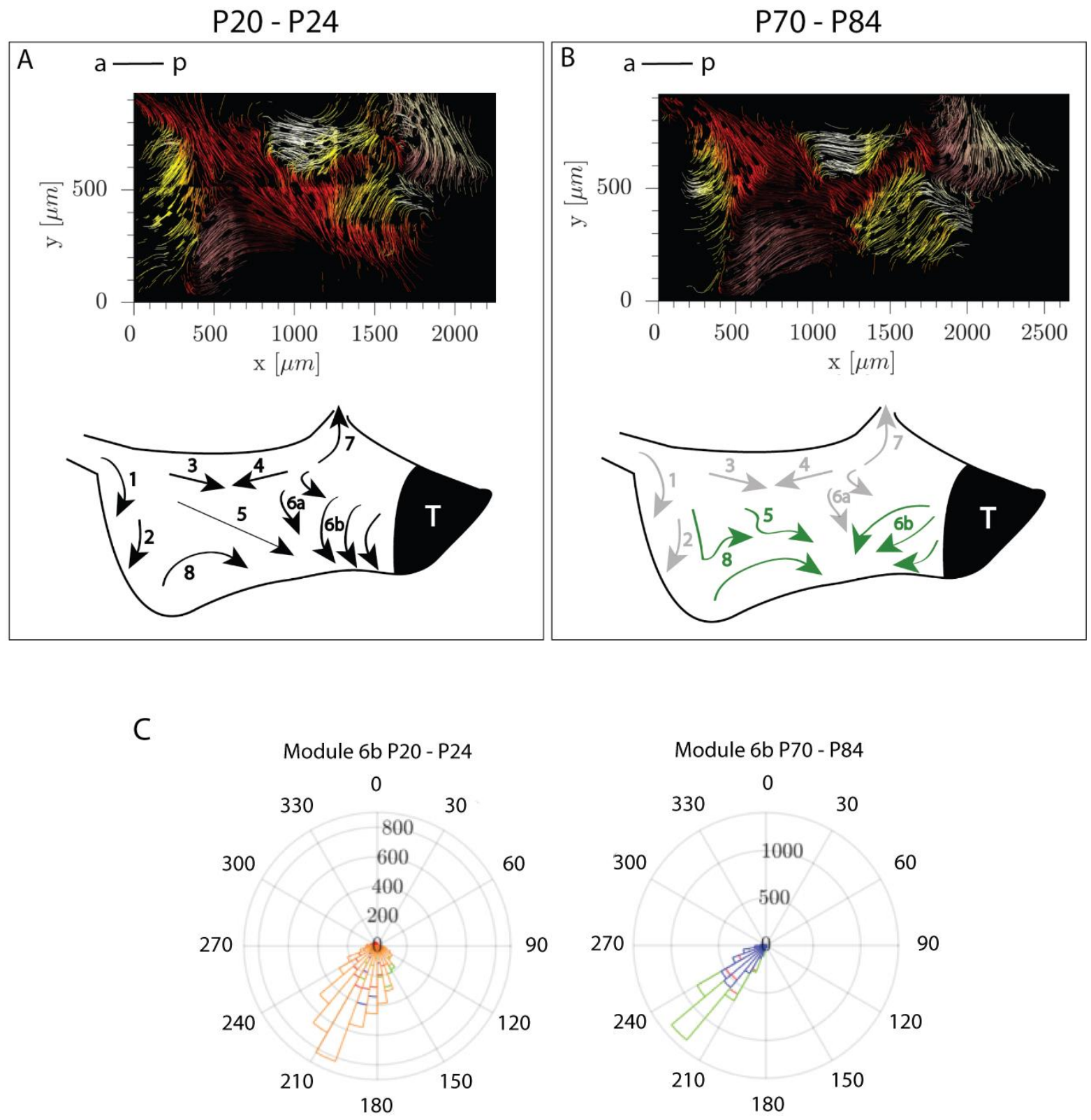

Fig. 28: V3V flow pattern of young and mature mice changes. (A) The v3V in 3 Weeks old mice displays the 8 modules. (B) The v3V flow pattern of adult mice shows changes in flow directions in modules 5,6, and 8. (C) Circular plots of the bead-track direction show how the flow direction of module $6 \mathrm{~b}$ ranges from $120^{\circ}$ to $260^{\circ}$ in mice at $\mathrm{P} 20-24$ but at $\mathrm{P} 70-\mathrm{P} 84$ it is directed towards $200^{\circ}-260$.

\subsubsection{Timed change of ciliary beating direction in module $6 \mathrm{~b}$}

One of the differences in the $\mathrm{v} 3 \mathrm{~V}$ flow pattern of young and adult mice is a change of ciliary beat direction in module $6 \mathrm{~b}$ from towards the tanycyte region to away from it. Furthermore, once the cilia beat direction in module $6 \mathrm{~b}$ is changed, it is maintained throughout life (Fig. 29). We chose this area of the v3V to further examine how these changes are carried out. To give a 
higher temporal resolution of the reorientation of flow pattern, we generated flow maps of the v3V whole mounts of juvenile mice at different ages between P28 and P42. From previous experiments, we had observed that at P20 - P24 the flow in module $6 \mathrm{~b}$ starts at the anterior border of the tanycyte region, bends ventrally in an anticlockwise manner and then flows towards the tanycyte region. This pattern is maintained until around P32, the exact time can vary slightly from animal to animal. However, between P33 - P35, we observe an intermediate stage of the change in flow direction. At this age, the flow starts at the anterior border of the tanycyte region but then leads to the ventral edge of the v3V boundary. Latest after P36, this flow pattern changes in all mice. Although the flow starts in the same source reagion as in younger mice, after P36, the flow of module $6 \mathrm{~b}$ leads away from the tanycyte region towards the anterior $\mathrm{v} 3 \mathrm{~V}$ and meets the flow of module 5, 6a, and 8. Once the change in flow direction has occurred, it is maintained this way throughout life (Fig. 29). Interestingly, this change of flow direction only occurs in the left hemisphere, while the right hemispheric v3V remains unchanged. This means that in a closed ventricle, the right and left hemispheric flow patterns of module $6 \mathrm{~b}$ lead to opposite directions. 

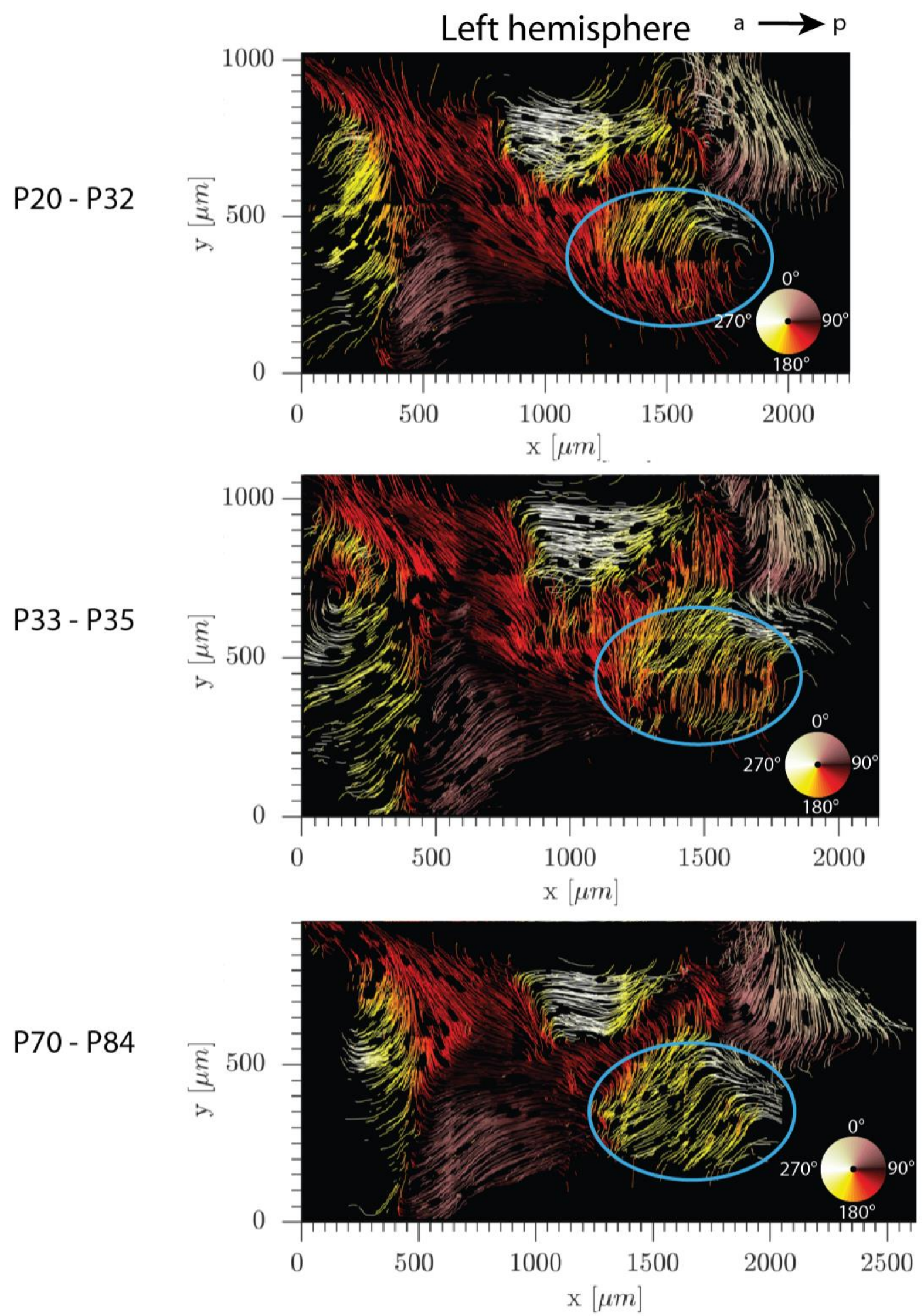

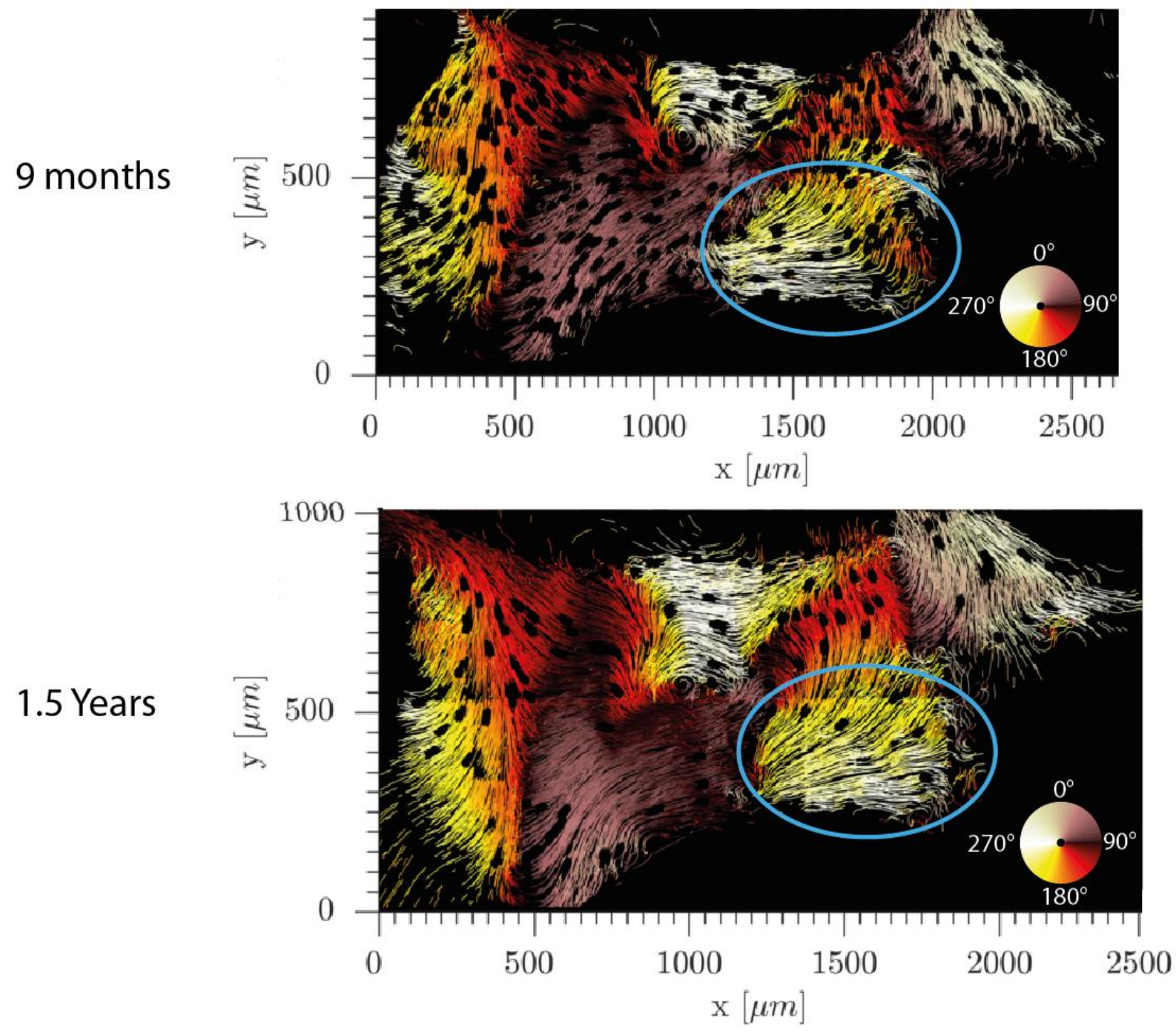

Fig. 29: Flow maps of mice at different ages, visualizing the reorientation of flow direction in module $6 \mathrm{~b}$, marked with a blue circle. V3V flow map at P20 - P24 displays how the flow pattern in module $6 \mathrm{~b}$ starts at the anterior side of the tanycyte region, turns anti clockwise and continues towards the tanycyte region. At P33 - P35 (the exact day can differ from animal to animal) we observe an intermediate state of the flow reorientation in module $6 \mathrm{~b}$. In adult mice (P70 - P84), this module displays a reorientation of the flow, where it starts in the anterior region of the tanycytes and leads away from the tanycytes. The changed flow pattern of module $6 \mathrm{~b}$ is maintained throughout life, which is visualized in the flow pattern of a v3V from a 9 months and a 1.5-year-old animal.

In conclusion, the $\mathrm{v} 3 \mathrm{~V}$ flow pattern gets first determined at P21, but for the first time, we show that between P33 - P35, it undergoes changes in flow direction in module $6 \mathrm{~b}$ of the left hemispheric v3V only. Before P33, the flow pattern of left-hemispheric module $6 \mathrm{~b}$ leads towards the tanycyte region, whereas by P36, it leads away from it. This may mean that the $\mathrm{v} 3 \mathrm{~V}$ flow pattern before P33 is designed to bring solutes to the tanycytes, which would be reduced after P36. 


\subsubsection{Period involvement}

We have established that the above described processes of the change in the flow pattern of module $6 \mathrm{~b}$ are timed precisely. As it is known, PCP does not change once established, thus we hypothesize that new ependymal cells form postnatally and are polarized in the opposite direction than those that were formed prenatally in order to change the near-wall flow in module 6b. Evidence of this is provided by the fact that ependymocytes are able to renew $(29,153,154)$. The combination of a timed event that may involve postnatal cell division drew our attention to Period that is a known regulator of postnatal cell cycle events in mammals (140). The orthologue of this gene in C. elegans, Lin42, regulates the timed developmental processes such as germline development or molting $(142,144,155)$.

To investigate whether the timed event of the change in flow direction in module $6 \mathrm{~b}$ is Period dependent, we created v3V flow maps of Per1 ${ }^{-/} \mathrm{Per}^{-/}$double knockout mice. We observed that the flow pattern of adult mice is comparable to that of wild type mice at the age of P20 - P24. All 8 modules develop as they do in wild type individuals but the change in the flow direction of module $6 \mathrm{~b}$ after P36, is absent in the mutants. Subsequent staining of Odf2 and centriolin that enables the visualization of the rotational polarity of cilia bundles further confirmed the lack of change in flow direction (Fig. 30).

Here we show that age-dependent changes of the v3V flow directions are directly or indirectly Period dependent. We hypothesize that this function of Period is related to the timing of a postnatal developmental process that involves cell division. 

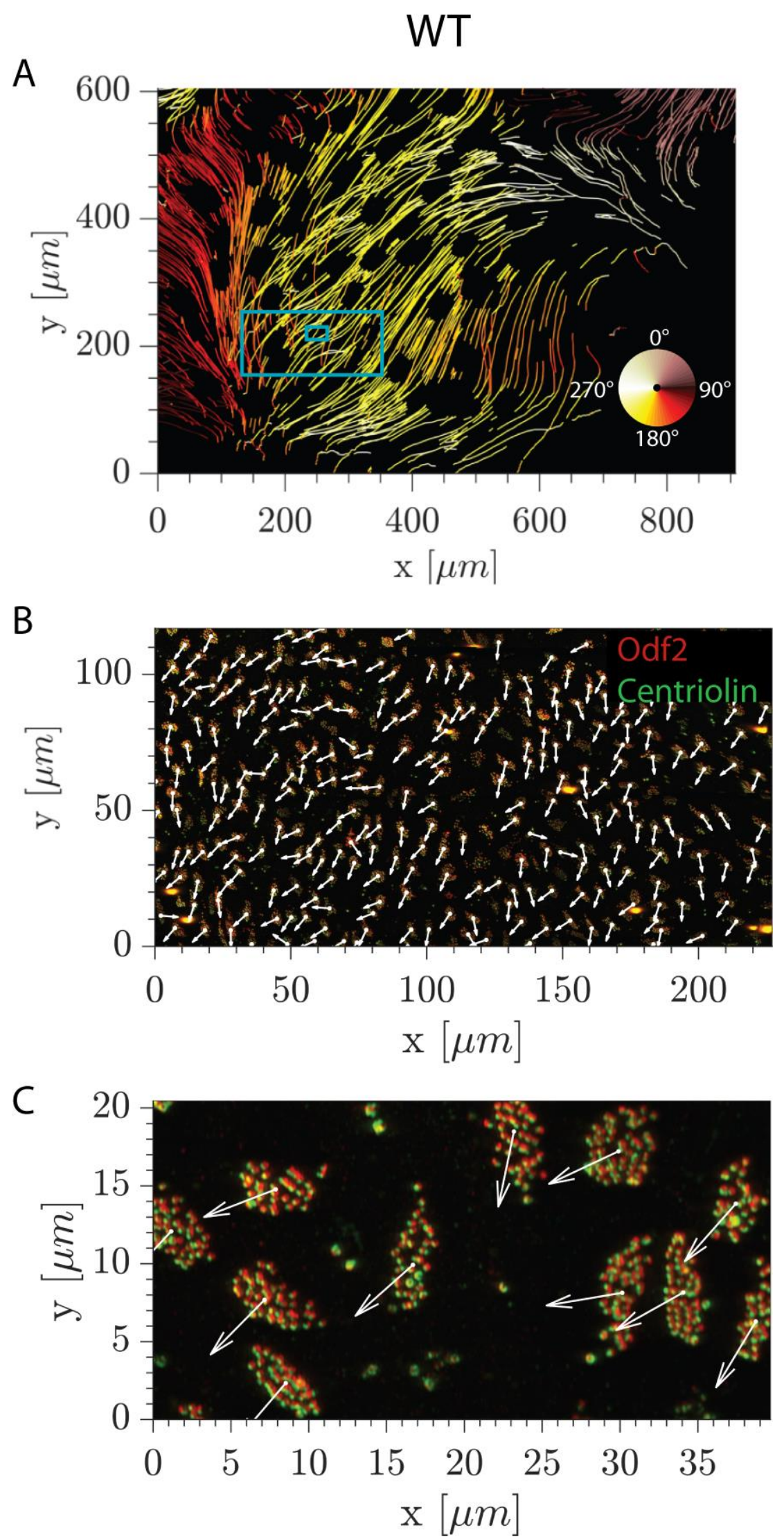

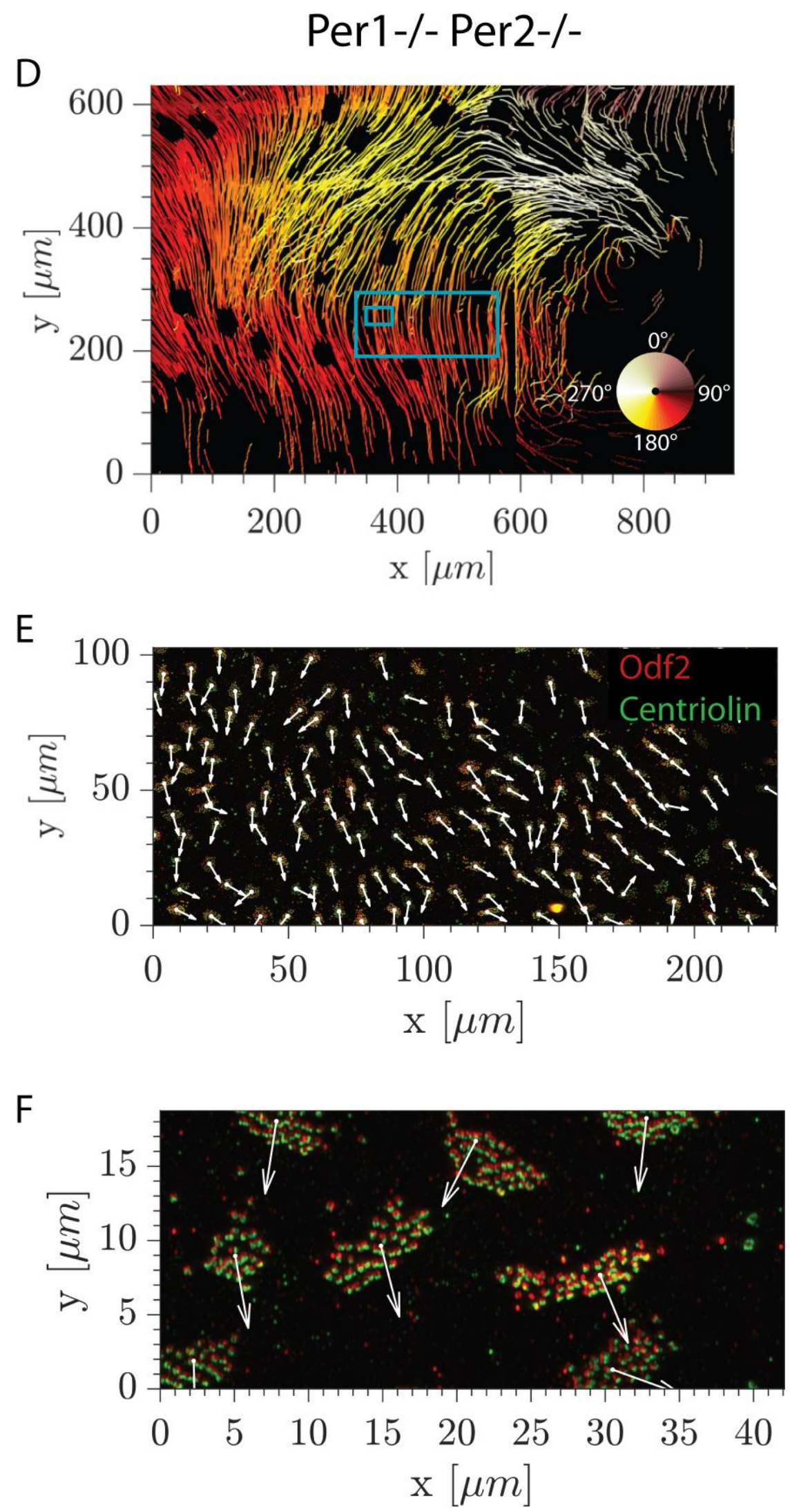

Fig. 30: Per1Per2 involvement in the flow change of module 6b. (A) Flow-pattern of module $6 \mathrm{~b}$ in a wild type mouse at P36. Blue squares indicate the position for which the rotational polarity is shown in B and C. (D) Flow-pattern of module $6 \mathrm{~b}$ in

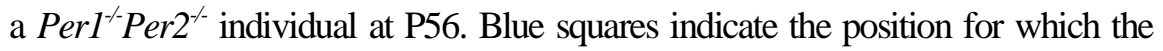
rotational polarity is shown in $\mathrm{E}$ and $\mathrm{F}$. 


\subsubsection{Preliminary results for future investigation}

\subsubsection{Rotational polarity during changes in flow pattern}

In section 3.1.3 we showed that the rotational polarity is correlated with the flow pattern. This indicates that a change in flow pattern must also involve changes in the rotational polarity of the underlying cilia. To confirm this, we generated v3V flow maps of mice at P35, which displayed the intermediate state of the change in flow direction in module $6 \mathrm{~b}$. Then, we stained the whole mounts for Odf2 and centriolin. This way we revealed the rotational polarities of the cells during the reorientation. We observed that a number of ependymal cells do not display aligned rotational polarities within the bundle, which may be in the process of changing the beating direction of their cilia (Fig. 31).

In wild type animals, disorganized cilia inside a bundle are only observed, when basal bodies have docked on the apical surface during ependymal differentiation before they are aligned by PCP signaling $(20,117)$. Furthermore, the fact that the circadian clock gene Period is involved in this change, we are encouraged to hypothesize that the reorientation of cilia beating involves the formation of new ependymal cells (138). 

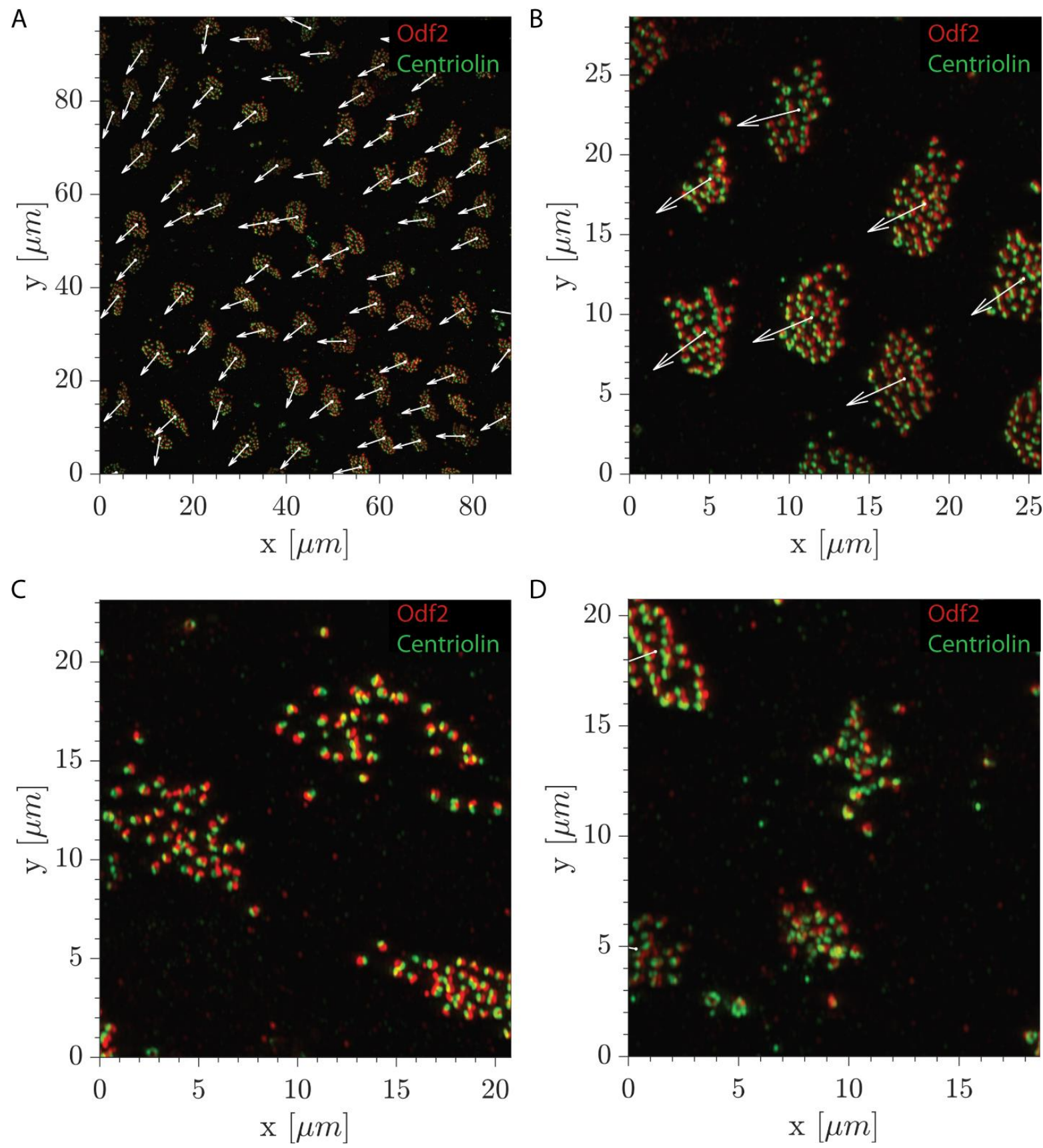

Fig. 31: Module 6b stained for Odf2 (red) and centriolin (green) during the reorientation of the ciliary beating direction at P35. (A) An overview of the average rotational polarities in cells of module 6b. (B) Cilia bundles of the module $6 \mathrm{~b}$, directed away from the tanycyte region. (C) Depitction of cilia bundles, where ciliary rotational polarity is not aligned. (D) Cilia bundles, which seem to either be in the process of degeneration or docking. 


\subsubsection{KI67 stain indicates cell division}

We showed that around P35 a number of cilia display non-aligned rotational polarities within a bundle. And together with the observation that Period may be related to this event, we hypothesize that in this region new ependymal cells are formed. To confirm this, we stained the $\mathrm{v} 3 \mathrm{~V}$ with the mitosis marker KI67 and checked its localization on the v3V. We found the presence of some KI67 positive cells in module 6b (Fig. 32). Only a few dividing cells are visible in module $6 \mathrm{~b}$, which may result from the fact that the immunolabeling of dividing cells only occurs if, before fixing the tissue, cells were dividing. This indicates that KI67 staining is probably not the most suitable method to investigate proliferation. For further investigation, we plan BrdU labeling of proliferating cells between P28 - P35.

Nonetheless, KI67 positive cells indicate that the change in flow direction in module $6 \mathrm{~b}$ could depend on the formation of new cells. It is yet to quantify, how high the ependymal turnover may be at this age and how the $P e r 1^{-/} P e r 2^{-/-}$mutants are affected.

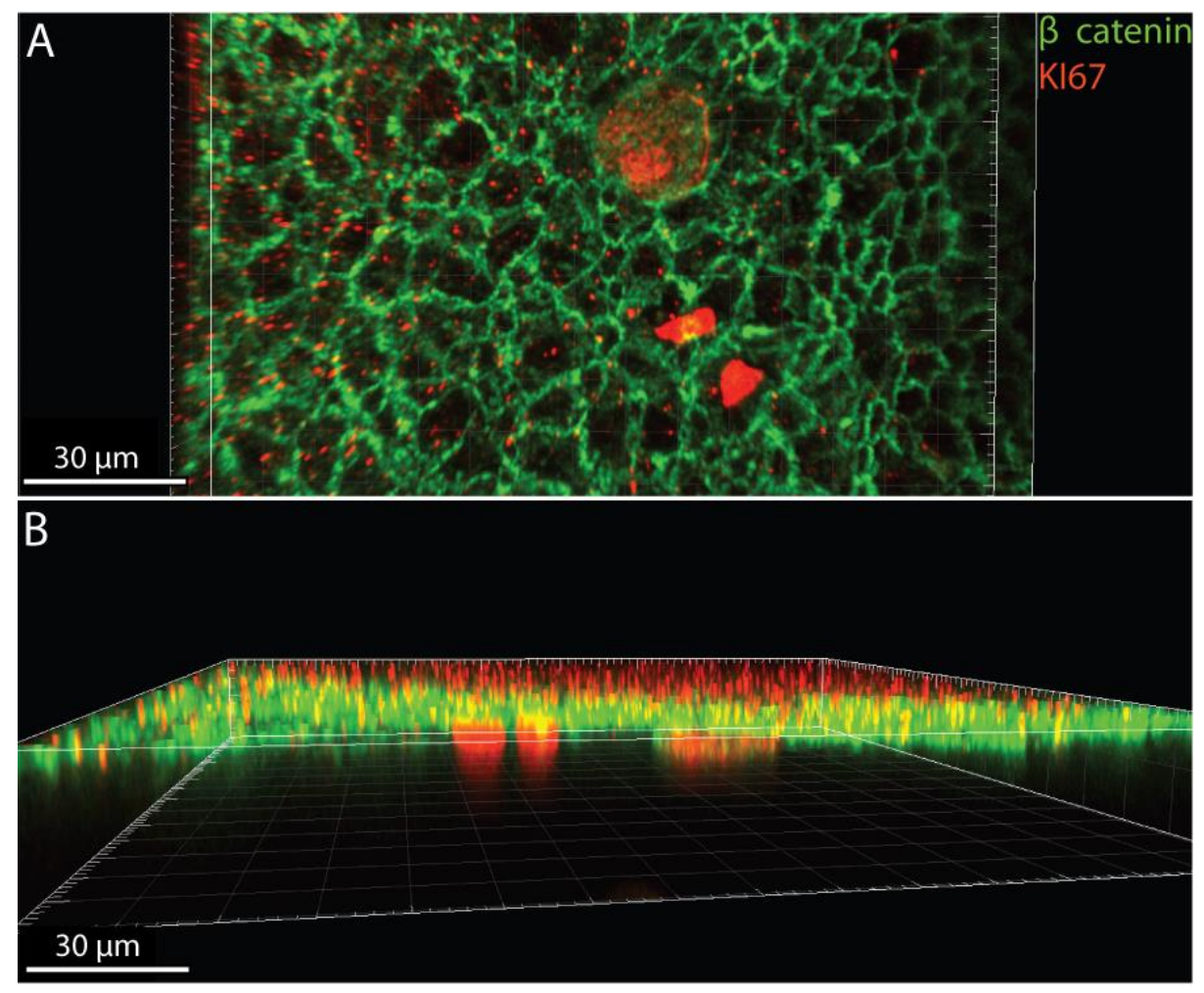

Fig. 32: Module 6b stained for beta catenin (green) and the mitosis marker KI67 (red) during the reorientation of the ciliary beating direction. (A) Top view of a 3D stack showing three Ki67 positive cells in module 6b. (B) Side view of the stack in (A) shows that Ki67 positive cells are located within or just beneath the ependymal plane. 


\section{Discussion}

\subsection{PCP gives rise to the complex flow pattern of the $\mathrm{v} 3 \mathrm{~V}$}

Flow maps based on particle tracking were recorded and revealed a complex flow pattern in the $\mathrm{v} 3 \mathrm{~V}(18)$. These patterns were obtained from $\mathrm{v} 3 \mathrm{~V}$ explants that were dissected from the brain and within a few minutes placed into culture medium. It remained to establish that the observed flow directions seen in this material reflect flow directions in the living animal. A way to address this issue is the analysis of translational and rotational polarities in the ependyma of explants. It is a central dogma of the planar cell polarity concept that these polarities do not change, at least not on the time scale of minutes (see Introduction). To determine translational polarity, v3V explants were stained for $\beta$ catenin that detects adherens junctions and thus marks cell boundaries. In addition, $\gamma$ tubulin staining of the basal body was used to define the position of the cilia bundles (see Results). Together this information allows one to define the translational polarity of each cell and thus one can predict cilia beating direction in cilia bundles and hence flow direction. Rotational polarity was determined by centriolin staining of the basal feet and Odf2 staining of the basal body (see Results). With this information, one can predict the beating direction of individual cilia. The beating direction predicted by translational and rotational polarities were then compared with the bead-tracking-based flow maps.

Since each wall of the v3V consists of approximately 20,000 ependymal cells, and because several hundred samples were measured, novel methods for data collection and data analysis were developed that deliver reliable information on cell polarity, basal foot orientation, cell size, and cilia bundle shape.

\subsubsection{Translational and rotational polarity presage the flow pattern}

The correlation of the bead track directions with the translational polarity directions in the $\mathrm{v} 3 \mathrm{~V}$ showed that the translational polarity of ependymal cells match quite well and thus translational polarity presages the flow pattern (Fig. 32). Taking the data of three mice, in module1, the angle of bead tracks/flow ranges between $110^{\circ}$ to $190^{\circ}$ and the translational polarity covers a range of $60^{\circ}$ to $230^{\circ}$. In module 2 , the range encompasses $190^{\circ}$ to $300^{\circ}$ for the flow and $100^{\circ}$ to $260^{\circ}$ for the translational polarity. The opposing flows of module 3 and 4 show strong correlation 
with translational polarity. The bead tracks of these modules range from $90^{\circ}$ to $180^{\circ}$ and $200^{\circ}$ to $320^{\circ}$, respectively, The corresponding translational polarity directions lie between $40^{\circ}$ to $180^{\circ}$ (flow3) and $160^{\circ}$ to $330^{\circ}$ (flow 4), respectively. In module 5, the flow direction extends between $60^{\circ}$ to $230^{\circ}$ in rather good agreement with the translational polarity directions from $0^{\circ}$ to $270^{\circ}$. The reflex angle of flow pattern and the broad polarity direction in the region of flow 5 is due to the fact that at this location whirls will form and, moreover, modules $3,4,6 \mathrm{a}, 6 \mathrm{~b}$, and 8 are directly bordering to flow 5 . The translational polarity directions of modules $6 \mathrm{a}, 7$, and 8 range from $40^{\circ}$ to $190^{\circ}, 240^{\circ}$ to $100^{\circ}$, and $350^{\circ}$ to $130^{\circ}$, respectively. The corresponding flow directions range from $100^{\circ}$ to $220^{\circ}, 320^{\circ}$ to $80^{\circ}$, and $40^{\circ}$ to $110^{\circ}$.

A noticeable divergence between the flow directions and translational polarity directions characterizes module $6 \mathrm{~b}$. Flow direction ranges from $200^{\circ}$ to $270^{\circ}$ but translational polarity encompasses a much wider angle $\left(60^{\circ}\right.$ to $\left.270^{\circ}\right)$. This widespread distribution may originate from the fact that in module $6 \mathrm{~b}$ ependymal cells are interspersed with tanycytes $(35,156)$. Tanycytes have but 1 or 2 primary, non-motile cilia that are not displaced from the center of the cell as is the case for motile cilia bundles. This is likely to introduce a significant error in determining translational polarity. As we will discuss below, the flow of module $6 \mathrm{~b}$ undergoes changes in flow direction. This change may produce additional complexities in translational polarization of this area that may cause a wider spread of angular distribution. We conclude that largely the directions of the bead tracks and translational polarity point in the same direction for most v3V modules.

This conclusion is further reinforced by our finding that the rotational polarity of cilia bundles in the $\mathrm{v} 3 \mathrm{~V}$ also correlates with the flow pattern. The quantification of rotational polarity in adults focused on modules 3, 4 and whirls, but in some specimen modules 1, 2 and $6 \mathrm{~b}$ were also quantified. Rotational polarity in the straight flows of module $1,2,3,4$, and $6 \mathrm{~b}$ range from $80^{\circ}$ to $170^{\circ}, 170^{\circ}$ to $280^{\circ}, 60^{\circ}$ to $160^{\circ}, 190^{\circ}$ to $340^{\circ}$, and $100^{\circ}$ to $300^{\circ}$, respectively (Fig. 32 ). In the separatrix, where flows 3 and 4 meet, the rotational polarity directions cover a wide range from $80^{\circ}$ to $300^{\circ}$. In all cases, the rotational polarity of cilia bundles presaged the flow pattern. Of particular interest are the whirls that form at different places within the v3V. Such whirls encompass up to 100 ependymal cells often with poorly defined translational polarity and disorganized expression of PCP factors (see below). However, it was possible to accurately determine rotational polarity. For clockwise and anticlockwise whirls, rotational polarity did correlate with the flow direction. The whirl-creating ependymal cells exhibited, from one cell 
to the next, a gradually changing rotational polarity, eventually forming either clockwise or anticlockwise full circle.
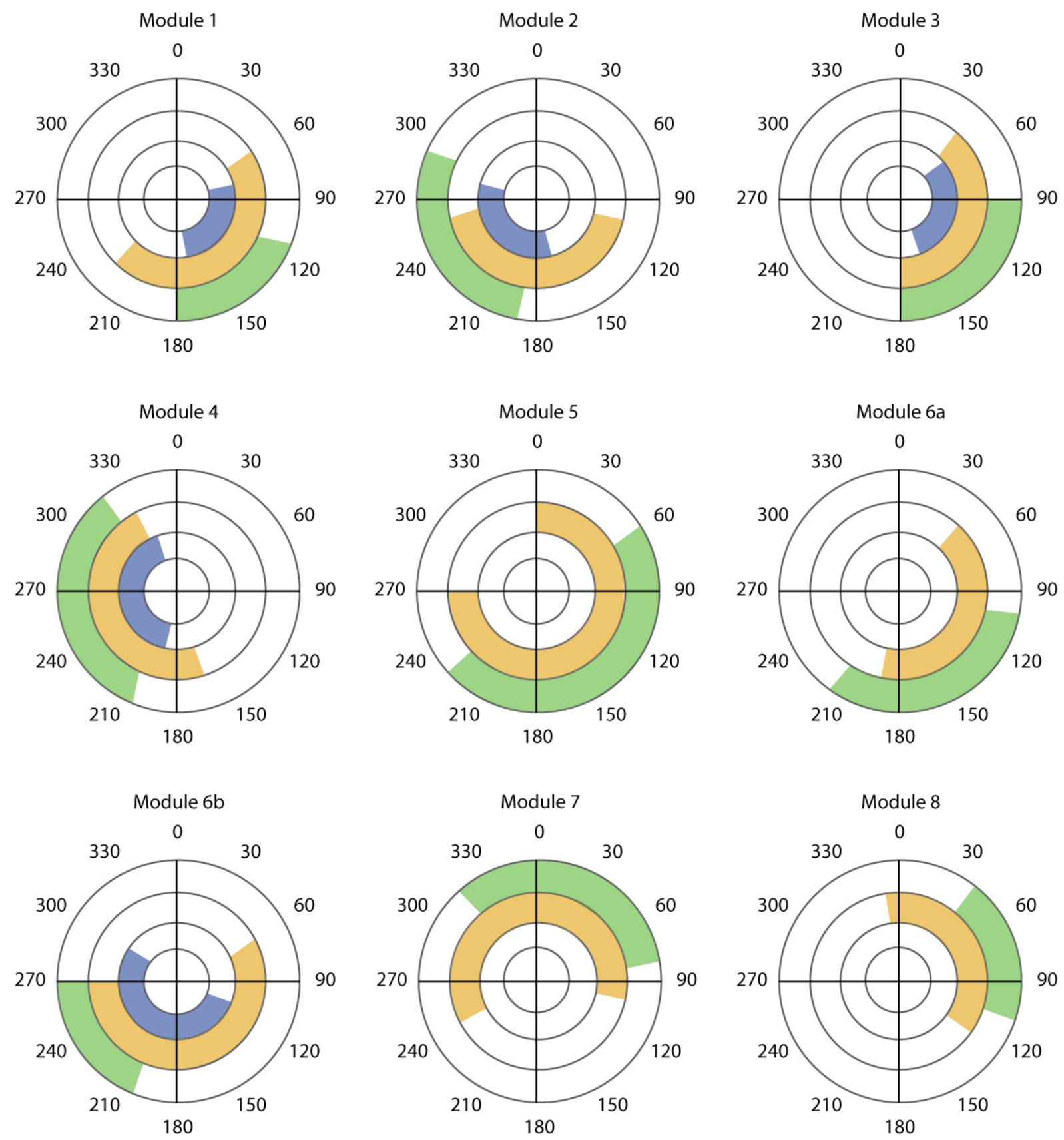

Rotational polarity

Translational polarity

Flow direction

Fig. 33: Schematic summary of the directions of rotational polarity (blue), translational polarity (orange), and flow (green) in all modules of the v3V. 
We set out to investigate whether the complex flow patterns seen in ventricular explants are occurring in the natural environment of the brain of a living animal. We showed that ependymal cells of the $\mathrm{v} 3 \mathrm{~V}$ exhibit translational and rotational polarities that presage the flow pattern that is observed in the explants. We infer from this data that the in vivo flow pattern resembles the one that is seen in explants. The sole caveat is that in the brain, the tissue forms a cavity and the two ependymal walls are in close proximity (50 to $200 \mu \mathrm{m})$. It is thus possible that flows of the left and the right wall influence each other in vivo.

In the brain, PCP proteins underlie translational and rotational polarities that then control the beating direction of motile cilia, and thereby create the observed flows. Thus, the next step in the research is to localize PCP proteins in individual flow modules.

\subsubsection{Vangl1 expression correlates with the flow pattern}

Among the proteins implicated in the establishment of module polarity is the transmembrane protein Vangl1 that is one of several PCP proteins (see Introduction). Immunohistochemical analysis shows that Vangl1 expression in the $\mathrm{v} 3 \mathrm{~V}$ is a canonical expression pattern also seen in other tissues and appears as a row of separate or interlinked "chevrons" (109). For example, in module 1 that initiates at the entrance of the $\mathrm{v} 3 \mathrm{~V}, \operatorname{Vang} 11$ immunoreactivity is observed at that edge of the ependymocyte that faces the source of this flow, which is at the entrance into the v3V. Precedence from other tissues such as trachea states that cilia beat away from the Vangl1positive edge $(109,157)$. This would mean that flow 1 initiates at the inlet of the v3V. and progresses into the ventricle. This is what is actually observed in videos of beating cilia and in the flow maps (18). Ependymocytes of module 2 and $6 \mathrm{~b}$ show Vangl1 expression that is restricted to that edge of the cell that faces the source of flows 2 and 6b, respectively. Consistent with the rule that cilia beat away from the Vangl1-positive edge, one would predict a flow in an anterior-ventral direction, which is what is observed in the flow map. In flow module 7 , Vangl1 is expressed on that side of the cell, which faces the tanycyte region. This would predict a flow that starts just dorsally of the tanycyte region and progresses to the outflow duct of the $\mathrm{v} 3 \mathrm{~V}$. This prediction is correct. In summary, the chevron-type expression motif locates to those edges of the ependymocyte that faces the source of the flow. Since the v3V has numerous flows and therefore also multiple sources, the Vangl1 expression pattern is complex.

The impression of complexity is also illustrated in the separatrix or in the regions of whirls, where flows from different sources meet. Vangl1 expression in the separatrix is confined to 
specific edges of the ependymocytes. However, the resulting chevrons point in different directions. Even in the whirl-forming ependymal cells edges are stained and may form chevrons. Our rotational polarity analyses show that cilia bundles do not beat in the same direction in whirls and there are multiple tangential sources of fluid. Thus, the whirly fluid flow correlates with the intricate Vangl1 expression pattern.

\subsection{Predetermined cellular morphology across the v3V}

The systematic and high throughput analysis of ependymal explants yielded several unexpected insights regarding the ependymal cell geometry. For thousands of ependymal cells across the $\mathrm{v} 3 \mathrm{~V}$, we computed the size of the apical surface area, the size of the apical area covered by cilia, the length of the vector drawn from the cell centroid to the cilia bundle centroid $(\overrightarrow{C C})$, and the shape of the cilia bundles. Our observation shows that although v3V cells have heterogeneous morphologies, they follow a stereotypical pattern that varies very little between animals. In this pattern, cells with the largest apical area sizes, the largest cilia bundles, and the longest $\overrightarrow{C C}$ vectors show the same distribution pattern in the $\mathrm{v} 3 \mathrm{~V}$. The following text discusses this point and will solely focus on the size of the apical area. This area was classified as "large cells" $\left(>150 \mu \mathrm{m}^{2}\right)$, "intermediate cells" $\left(80-150 \mu \mathrm{m}^{2}\right)$, and "small cells" $\left(40-80 \mu \mathrm{m}^{2}\right)$. Cells below $40 \mu \mathrm{m}^{2}$ surface and with a cilia bundle size below $15 \mu \mathrm{m}^{2}$ are mainly tanycytes, that are located in the most posterior area of the v3V. Fig. $33 \mathrm{~B}$ summarizes the distribution of large and small cells. Noteworthy, are the three long strands of large cells in the rostral part of the $\mathrm{v} 3 \mathrm{~V}$ and the broad diagonal band of large cells passing through the middle of the v3V. Flow 8 transects the three stands and terminates in the lower part of the diagonal band. Other flows also extend across populations of large and small cells. Flow 4 mostly encompasses large cells, while flow 3 is driven mostly by small cells. Thus, the separatrix is generated by cells at the opposite ends of the size spectrum.

The eccentricity of the cilia bundles, in which the value 0 represents a circular cilia bundle and 1 a bundle in the shape of a line, follows a pattern in the v3V (Fig. 33 C). No cilia bundles with an eccentricity below 0.5 are found in the $\mathrm{v} 3 \mathrm{~V}$. Most small cells seem to possess cilia bundles that are elongated (>0.8), except for some areas in module 8. Cilia bundles of this shape are also in a region that covers module 1 and 2 . In the diagonally oriented group of large cells are two domains of highly eccentric cilia bundles. One is located dorsally, beginning from the anterior limit of module 4 and extending ventrally towards flow 5. The second group lies at the 
posterior edge of the diagonal band. In the area of module $6 \mathrm{~b}$, where cell sizes are variable, the cilia bundle shapes are also variable.

A

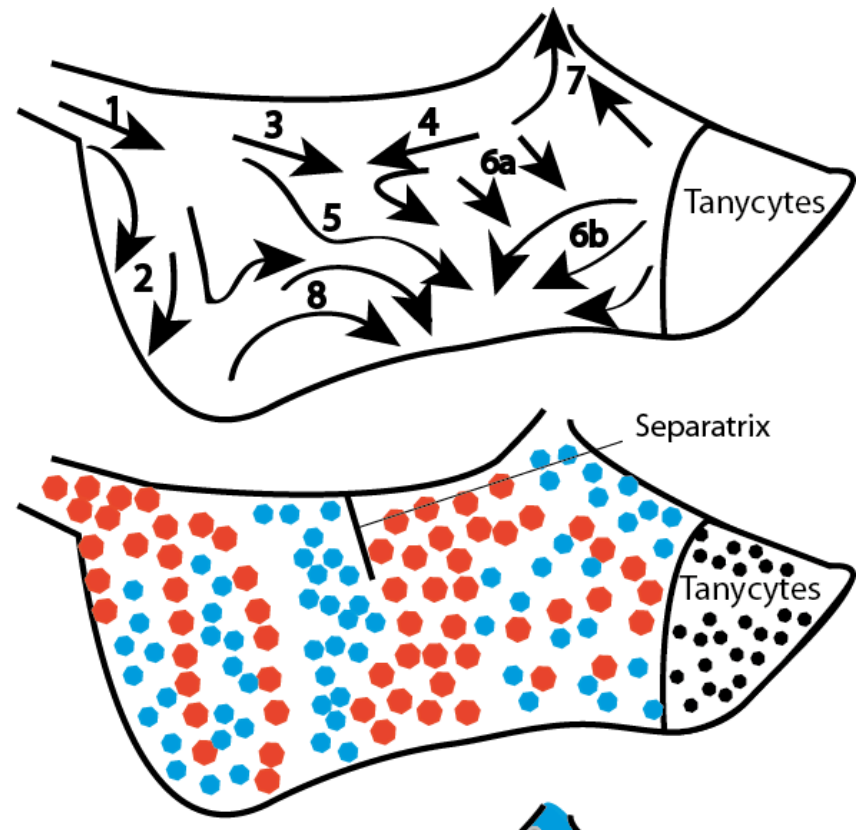

C

B

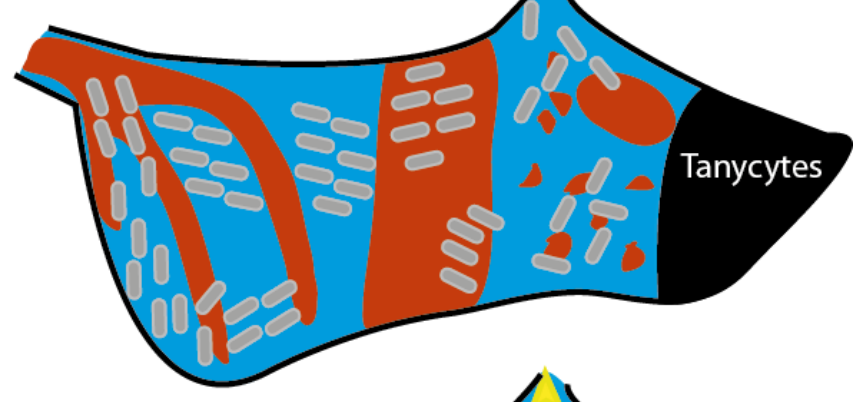

D

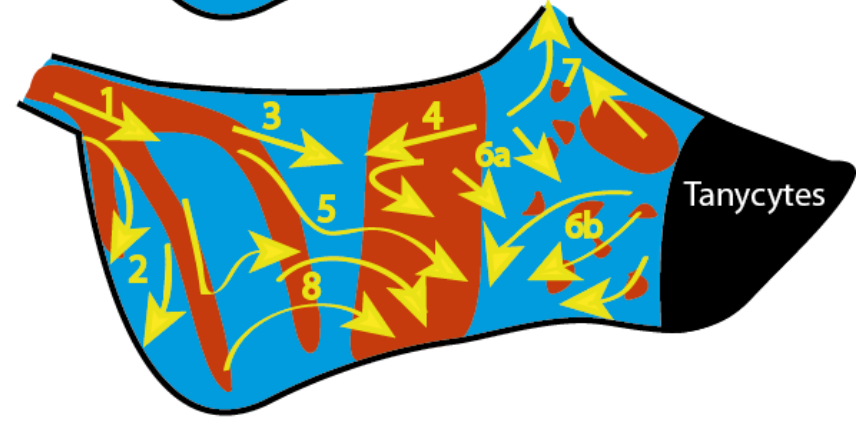

- Large ependymal cells

- Small ependymal cells

Highly eccentric cilia bundles (>80)

Fig.34: Scheme of v3V flow pattern in comparison to its architecture. (A) Scheme of the v3V flow pattern. (B) V3V pattern of large (red) and small (blue) cells. (C) Pattern of highly eccentric cells in the v3V. The background of this scheme illustrates the pattern of large (red) and small cells (blue). (D) V3V flow pattern in yellow with regard to large and small cells depicted in the background. 
Why are the geometrical properties of ependymal cells invariant between animals? We found little evidence of a direct link between cell geometry and flow directions. It should be recalled that in addition to driving CSF flow, the ependyma is key tissue that separates CSF from the brain. This separation is leaky in the sense that CSF and interstitial fluid exchange solutes $(10,158)$. Thus CSF solutes, transported by the flows, could interact with the non-ciliated, microvilli-covered apical surface of ependymocytes and eventually end up in subependymal tissue. Such a mechanism has been proposed for tanycytes (159). Ependymocytes are interconnected by adherens junctions that allow passage of molecules in either direction. Compared to large cells, clusters of small ependymocytes show a disproportionate increase of intercellular substance exchange per unit area. How the pattern of cell size distribution is established remains completely unknown. It is likely that factors that control the size of cells are involved. Examples for such factors are the IGF/PI3K/AKT/mTORC1 pathway and the transcription factor Myc $(160,161)$. The transcription factor Myc increases the production of ribosomal RNA (rRNA), the size of nucleoli and protein synthesis. Furthermore, it is associated with the regulation of metabolic activity within a cell that contributes to the control of cell size $(161,162)$. The IGF/PI3K/AKT/mTORC1 pathway is activated in response to external stimuli such as oxygen, stress, amino acids, ATP levels, and insulin growth factor 1 (IGF1). IGF1 mediated activation of mTORC1 causes a cellular response that encompasses an increased lipid and protein biosynthesis and inhibition of autophagy, which in turn increase growth $(160,162)$. Interestingly, IGF1 is one of the factors that is produced by the choroid plexus and could be part of the passive CSF flow that exists before RGCs differentiate into ependymal cells $(3,27)$.

\subsection{Age-dependent changes in the v3V flow pattern}

Developmental studies of the lateral ventricle show that multiciliated cells emerge shortly after birth $(20,134)$. There are no published developmental studies on the third ventricle, but preliminary analysis of P5 and P7 tissue suggest a similar temporal profile (SK, unpublished data). Our main effort with regard to time-dependent changes in the ependyma has concentrated on male mice at the age of P21 and older. We observed two types of changes in the flow pattern. First, over the course of the murine life, clockwise and counterclockwise whirls emerged at specific sites. Second, between P33 - P35 the flow pattern in the in modules 5, 6b, and 8 changes. This change, as well as whirl formation, differ between the left and the right v3V walls. 
Young mice (P21 to P24) rarely show the characteristic central whirl below the separatrix (Figure in Results), whereas mice older than 9 months always possess an anticlockwise central whirl at this location. It should be noted that even prior to whirl formation at the base of the separatrix, flows 3 and 4 change direction by making a 150 degree leftward turn towards the tanycyte region. This turn could eventually develop to a full reversal in direction and thereby form the whirl. This elaborate change in direction resulting in a whirl could either be the result of hydrodynamic forces (134) or a reorientation of the rotational polarity of those ependymal cells that generate the whirl. It is also possible that new cells emerge at this site that adjust their cilia beating direction to a preexisting flow (134). Fact is that the group of ependymal cells that drive this new whirl show the appropriate rotational polarity (see above). After the age of around 6 months, we frequently observe clockwise whirls at the junction of module $6 \mathrm{~b}$ and 8 and sometimes at the entrance of $\mathrm{v} 3 \mathrm{~V}$.

Between P33 and P35 the flow pattern in the ventral area of the left hemispheric v3V changes in modules 5, 6b, and 8. The most prominent change is that in flow module $6 \mathrm{~b}$. Until P33 the flow in this module points in the direction the tanycyte region (i.e. delivers solutes to the tanycyte region) but two days later, flow6b leads away from this region (Fig. 26, 27). It is striking that these changes in the flow pattern are specific to the left hemisphere and take place at a particular time and in just a few days. The mechanism of this directional change is as yet unclear. However, preliminary data show that at P35 there are many cilia bundles that contain disoriented cilia, as judged by the variation in direction of the basal feet (rotational polarity). This could either mean that the cilia on existing ependymal cells are removed and new cilia form or that new ependymal cells are born, which form new cilia with a changed rotational polarity. We have evidence from mitotic marker Ki67 staining that nuclei in that module suggest cell proliferation. It should be noted that there is no evidence in the literature supporting the existence of proliferation of ependymal cells in $\mathrm{v} 3 \mathrm{~V}$. However, the ependyma in $6 \mathrm{~b}$ contains tanycytes that do proliferate when exposed to growth factor stimuli (159). It should also be pointed out that the flow pattern of module $6 \mathrm{~b}$ is governed by hundreds of ependymal cells. Thus a change of flow pattern is only observable when the majority of the cells beat in a different direction. 


\subsection{Period involvement in the timing of postnatal processes}

To proof that the flows directionally transport CSF-contained substances to specific sites within the ventricular system, one will have to genetically alter flow patterns. This should result in a physiological phenotype. Serendipitously, we discovered that $P e r 1^{-/} \mathrm{Per}^{-/-}$double mutant mice do not undergo the reorientation of flow direction in module $6 \mathrm{~b}$. The flow pattern in $\mathrm{Perl}^{-/}$ $\mathrm{Per}^{-/}$double mutants is comparable to that of wild type mice that are younger than P33. Thus in wild type mice flow $6 \mathrm{~b}$ orientation is different on the right and the left side wall of the $\mathrm{v} 3 \mathrm{~V}$ while in double mutants there is symmetry.

PERIOD (PER) is an inhibitory factor of the circadian clock blocking the function of the transcriptional activators BMAL1 and CLOCK. Thus PER is acting in a negative feedback and PER expression is controlled by the CLOCK and BMAL1 (163-165)). Per1Per2 double knockout mice lack a circadian clock (164). However, there is so far no evidence for Period's involvement in a postnatal timing-related processes, except that in Caenorhabditis elegans $(C$. elegans) the Period orthologue, lin42, times postnatal developmental processes (142-145). During larval development, $C$. elegans goes through four stages of molting. Lin 42 regulates the heterochronic miRNA expression in these stages and its mutation leads to premature terminal differentiation of hypodermal seam cells $(143,146)$. Period gene functions have also been demonstrated in the context of cell cycle control (138). In mammals, Period was shown to influence the expression of the cell cycle checkpoint gene pl6-link4A. In Per mutants, postnatal cell divisions such as in wound healing were perturbed $(139,140)$. Our preliminary results suggest that the formation of new ependymal cells is required for changing the flow direction in module 6. In $\mathrm{Perl}^{-/} \mathrm{Per}^{-/}$mutants this change does not occur or is retarded, possibly because cell proliferation is impaired. 


\section{Summary}

The ventricular system of the mammalian brain consists of the two lateral, a third, and a fourth ventricle that interconnect and are filled with circulating cerebrospinal fluid (CSF) (1). CSF streams along the ventricular walls propelled by bundles of motile cilia that protrude from the apical surface of wall-forming ependymal cells (14). Planar cell polarity (PCP), which is created by proteins that are asymmetrically expressed in ependymocytes, controls cilia beating direction $(113,166-169)$. PCP has two components referred to as translational and rotational polarity. Translational polarity is quantified by a vector $(\overrightarrow{C C})$ extending from the cell center to the center of the cilia bundle. Each cilium docks to a cylindrical, cytoplasmic basal body that extends a basal foot. The angular direction of the foot relative to the axis of the cylindrical basal body is referred to as rotational polarity $(19,120)$.

Explants consisting of flat-mounts of the ventral part of the third ventricle (v3V) show in ex vivo cultures very intricate flow patterns that are organized into eight modules. Within each of the modules, fluid is transported uniformly but flow directions differ between modules (18).

Aim 1 of this thesis was to investigate whether the flow pattern observed ex vivo reflects the in vivo flows. This was achieved by determining, throughout the $\mathrm{v} 3 \mathrm{~V}$, translational and rotational polarities and hence cilia beating directions. In addition, we analyzed the cellular expression pattern of the PCP protein Vangl1 in ependymocytes. By means of automated confocal microscopy and custom-made segmentation analysis, PCP and Vangl1 expression sites of $>20.000$ ependymocytes of the $\mathrm{v} 3 \mathrm{~V}$ wall were determined in multiple freshly sacrificed mice. Both, the localization of Vangl1 within ependymocytes and translational and rotational polarities presage modular an in vivo flow pattern in the $\mathrm{v} 3 \mathrm{~V}$ that strongly resembles that seen in ex vivo $\mathrm{v} 3 \mathrm{~V}$ explants.

Aim 2 was to search for complex features in the architecture of the v3V beyond the PCP. We computed the apical surface area of ependymocytes, the area and shape of cilia bundles, and the length of the $\overrightarrow{C C}$ vector. We discovered that ependymocytes of the cell morphology in the $\mathrm{v} 3 \mathrm{~V}$ are heterogeneous in (large and small cells, elongated and round cilia bundles etc.). However, cells of a given characteristic are found in the same location of the $\mathrm{v} 3 \mathrm{~V}$ of all mice analyzed. For example, there are elongated arrays of large surface area ependymocytes in the rostral part of the $\mathrm{v} 3 \mathrm{~V}$ that extend from the site of CSF influx to the ventral edge of the v3V. 
$\underline{\text { Aim } 3}$ was to search for temporal changes in the flow directions in the $\mathrm{v} 3 \mathrm{~V}$. We discovered that over the span of a mouse circular arrays of cilia driving a whirl-like flow emerge at particular sites. In addition, and specific for the left-side of the $\mathrm{v} 3 \mathrm{~V}$ wall, the flow pattern changes in several of the eight modules. A particular striking example was seen in the ventral part of flow 6. Prior to the switching, cilia beat towards the posterior end of the $\mathrm{v} 3 \mathrm{~V}$, while after the switch, they beat in the opposite direction. Interestingly, this switch would alter the CSF flow from one targeting the tanycytes to one leading away from the tanycytes.

Aim 4 was to identify genes that are involved in the temporal changes of CSF flow in the v3V. We made the important observation that the above-mentioned switches in flow directions do not occur in $\mathrm{Perl}^{-/} \mathrm{Per}^{-/}$double mutants. While wild type individuals develop a left-side asymmetry in the fifth week, in $\mathrm{Per}^{-/} \mathrm{Per}^{-/}$double mutants, the flow pattern remains symmetrical. Hence, on both walls flow persistently direct CSF towards the tanycyte region. Period genes are key regulators of circadian timing and influence a vast variety of physiological and pathophysiological processes in postnatal animals.

In conclusion, this thesis shows that the $\mathrm{v} 3 \mathrm{~V}$ architecture and the polarization of its cells is more complex than that of other tissues with multiciliated cells. This complexity is undoubtedly established during $\mathrm{v} 3 \mathrm{~V}$ development. We propose that the complex flow pattern suggests the existence of a novel way communication in the brain in which solutes are transported to particular regions by means of directionally beating cilia bundles. The timed switch of the orientation of cilia beating in flow 6 supports this hypothesis and shows that in each area planar polarity can be regulated independently from the rest. Furthermore, it exemplifies how ependymal development occurs in juvenile mice and may be controlled, at least in part, by circadian clock genes. 


\section{List of figures and tables}

\begin{tabular}{|c|c|}
\hline Figure 1 & Schematic illustration of the murine cerebral ventricles. \\
\hline Figure 2 & Flow maps of the $\mathrm{v} 3 \mathrm{~V}$, in which all modules are marked in blue arrows. \\
\hline Figure 3 & $\begin{array}{l}\text { Schematic illustration of hypothalamic nuclei that are located adjacent to the } \\
\text { v3V in the brain parenchyma. }\end{array}$ \\
\hline Figure 4 & $\begin{array}{l}\text { Schematic illustration of the ventricular wall in the posterior } \mathrm{v} 3 \mathrm{~V} \text {, where } \\
\text { ependymal cells meet tanycytes. }\end{array}$ \\
\hline Figure 5 & $\begin{array}{l}\text { Simplified scheme of the posterior } \mathrm{v} 3 \mathrm{~V} \text {, where tanycytes contact the brain } \\
\text { parenchyma. }\end{array}$ \\
\hline Figure 6 & Schematic illustration of axonemal cross sections of primary and motile cilia. \\
\hline Figure 7 & A simplified scheme of the localization of core PCP proteins. \\
\hline Figure 8 & $\begin{array}{l}\text { Schematic illustration of the translational and rotational polarity in ependymal } \\
\text { cells. }\end{array}$ \\
\hline Figure 9 & Schematic illustration of ependymal development in the lateral ventricle. \\
\hline Figure 10 & Illustration of the open book preparation to isolate the $\mathrm{v} 3 \mathrm{~V}$ \\
\hline Figure 11 & Summary of methods. \\
\hline Figure 12 & $\begin{array}{l}\text { The } \mathrm{v} 3 \mathrm{~V} \text { pattern of translational polarity directions correlate with the flow } \\
\text { pattern. }\end{array}$ \\
\hline Figure 13 & Circular plots of bead track directions in each module. \\
\hline Figure 14 & $\begin{array}{l}\text { For three wild type mice, circular plots show the quantification of translational } \\
\text { polarity directions of all cells within each module. }\end{array}$ \\
\hline Figure 15 & Comparison of human and computer designated $\overrightarrow{C C}$ vectors on ependymal cells. \\
\hline Figure 16 & Vangl1 expression correlates with the v3V flow pattern. \\
\hline Figure 17 & $\begin{array}{l}\text { The rotational polarity of all cilia in a bundle is visualized by staining the basal } \\
\text { body with Odf } 2 \text { and basal foot with centriolin. }\end{array}$ \\
\hline Figure 18 & $\begin{array}{l}\text { Comparison between the algorithm and human generated average rotational } \\
\text { polarity of a cilia bundle. }\end{array}$ \\
\hline Figure 19 & The rotational polarity presages the flow pattern. \\
\hline Figure 20 & Circular plots of rotational polarities. \\
\hline Figure 21 & $\begin{array}{l}\text { Maximum intensity projected, stitched, and segmented image, displaying cells } \\
\text { of the separatrix and module } 4 .\end{array}$ \\
\hline
\end{tabular}




\begin{tabular}{|c|c|}
\hline Figure 22 & Stereotypical pattern of cell sizes across the $\mathrm{v} 3 \mathrm{~V}$. \\
\hline Figure 23 & Stereotypical pattern of the size of cilia bundles. \\
\hline Figure 24 & Stereotypical pattern of the CC vector length distribution. \\
\hline Figure 25 & Stereotypical pattern of the eccentricity of cilia bundles. \\
\hline Figure 26 & Flow map of a v3V, isolated from a 6 months old mouse. \\
\hline Figure 27 & $\begin{array}{l}\text { A comparison between the area size of each module in young }(\mathrm{P} 20-\mathrm{P} 24) \text { and } \\
\text { adult (P70 - P84) mice. }\end{array}$ \\
\hline Figure 28 & V3V flow pattern of young and mature mice changes. \\
\hline Figure 29 & $\begin{array}{l}\text { Flow maps of mice at different ages, visualizing the reorientation of flow } \\
\text { direction in module } 6 \mathrm{~b} \text {. }\end{array}$ \\
\hline Figure 30 & Per1Per 2 involvement in the flow change of module $6 \mathrm{~b}$. \\
\hline Figure 31 & $\begin{array}{l}\text { Module } 6 \mathrm{~b} \text { stained for Odf } 2 \text { and centriolin during the reorientation of the ciliary } \\
\text { beating direction at P35. }\end{array}$ \\
\hline Figure 32 & $\begin{array}{l}\text { Module } 6 b \text { stained for } \beta \text { catenin and the mitosis marker KI67 during the } \\
\text { reorientation of the ciliary beating direction. }\end{array}$ \\
\hline Figure 33 & Schematic summary of the directions of polarization. \\
\hline Figure 34 & Scheme of v3V flow pattern in comparison to its architecture. \\
\hline Table 1 & $10 \times \mathrm{PBS}$ \\
\hline Table 2 & Primary antibodies \\
\hline Table 3 & Secondary antibodies \\
\hline Table 4 & Quantification of the occurrence of a central whirl. \\
\hline
\end{tabular}




\section{List of abbreviations}

\begin{tabular}{|c|c|}
\hline AHA & Anterior hypothalamic area \\
\hline Ankrd6 & Ankyrin Repeat Domain-Containing Protein 6 \\
\hline Arc & Arcuate nucleus \\
\hline AVPe & Anterio-ventral periventricular nucleus \\
\hline Bmal & Brain and Muscle Arnt Like \\
\hline Celsr & Cadherin EGF LAG Seven-Pass G-Type Receptor \\
\hline CNS & Central nervous system \\
\hline $\overrightarrow{C C}$ & Centroid to centroid vector \\
\hline Clock & Circadian Locomotor Output Cycles Kaput Protein \\
\hline CKIE & Casein Kinase $\varepsilon$ \\
\hline $\mathbf{C P}$ & Choroid Plexus \\
\hline Cry & Cryptochrome \\
\hline CSF & Cerebrospinal Fluid \\
\hline CT & Circadian time \\
\hline CVO & Circumventricular organ \\
\hline DD & 12 h dark : 12 h dark \\
\hline DM or DMH & Dorsomedial hypothalamic nucleus \\
\hline DMD & Dorsomedial hypothalamic nucleus, diffuse \\
\hline Ds & Dachsous \\
\hline DTM & Dorsal tuberomammillary nucleus \\
\hline Dvl & Dishevelled \\
\hline $\mathbf{E}$ & Embryonic day \\
\hline $\mathbf{F j}$ & Four-jointed \\
\hline Foxj 1 & Forkhead box protein $\mathrm{J1}$ \\
\hline $\mathbf{F t}$ & Fat \\
\hline Fzd & Frizzled \\
\hline ICV & Intracerebroventricular \\
\hline IFT & Intraflagellar transport \\
\hline ISH & In situ hybridization \\
\hline IP & Intraperitoneal \\
\hline LD & $12 \mathrm{~h}$ light : $12 \mathrm{~h}$ dark \\
\hline
\end{tabular}


LV

MCC

MM

MN

MnPO

MPOM

NMII

Npas2

OVLT

Per

$\mathbf{P}$

PCD

PCP

Pk

ADPKD

PVN

RHT

RGC

RMS

SCN

SVZ

TTL

Tyr-MT

V3V

Vangl

VMHDM

VMN

VMPO

VOLT

VZ
Lateral ventricle

Multi-ciliated cell

Medial mammillary nucleus, med

Medial preoptic nucleus

Median preoptic nucleus

Medial preoptic nucleus, med

Non muscle myosin II

Neuronal Pas Domain Protein 2

Organum vasculosum of the lamina terminalis

Period

Postnatal day

Primary ciliary dyskinesia

Planar cell polarity

Prickle

Autosomal dominant polycystic kidney disease

Paraventricular nucleus

Retinohypothalamic tract

Radial glial cell

Rostral migratory stream

Suprachiasmatic nucleus

Subventricular zone

Translational - transcriptional loop

Tyrosinated microtubules

Ventral third ventricle

Van gogh like

Ventromedial hypothalamic nucleus, dorsomedial

Ventromedial Nucleus

Ventromedial preoptic nucleus/

Organum vasculosum of the lamina terminalis

Ventricular Zone 


\section{Bibliography}

1. Swedenborg E. The Brain considered Anatomically, Physiologically and Philosophically. 1887 Volume 2

2. Lun MP, Monuki ES, Lehtinen MK. Development and functions of the choroid plexuscerebrospinal fluid system. Nat Rev Neurosci. 2015 Aug;16(8):445-57.

3. Johanson C, Stopa E, McMillan P, Roth D, Funk J, Krinke G. The distributional nexus of choroid plexus to cerebrospinal fluid, ependyma and brain: toxicologic/pathologic phenomena, periventricular destabilization, and lesion spread. Toxicol Pathol. 2011 Jan;39(1):186-212.

4. Pardridge WM. CSF, blood-brain barrier, and brain drug delivery. Expert Opin Drug Deliv. 2016 Apr 11;13(7):963-75.

5. Brightman MW, Reese TS. Junctions between intimately apposed cell membranes in the vertebrate brain. J Cell Biol. 1969 Mar;40(3):648-77.

6. Lun MP, Johnson MB, Broadbelt KG, Watanabe M, Kang Y-J, Chau KF, et al. Spatially heterogeneous choroid plexus transcriptomes encode positional identity and contribute to regional CSF production. J Neurosci. 2015 Mar 25;35(12):4903-16.

7. Milhorat TH, Hammock MK, Fenstermacher JD, Levin VA. Cerebrospinal fluid production by the choroid plexus and brain. Science. 1971 Jul 23;173(3994):330-2.

8. Oi S, Di Rocco C. Proposal of "evolution theory in cerebrospinal fluid dynamics" and minor pathway hydrocephalus in developing immature brain. Childs Nerv Syst. 2006 Jul;22(7):662-9.

9. Casaca-Carreira J, Temel Y, Hescham S-A, Jahanshahi A. Transependymal cerebrospinal fluid flow: opportunity for drug delivery? Mol Neurobiol. 2018 Apr;55(4):27808.

10. Hladky SB, Barrand MA. Mechanisms of fluid movement into, through and out of the brain: evaluation of the evidence. Fluids Barriers CNS. 2014 Dec 2;11(1):26.

11. Welton JL, Loveless S, Stone T, von Ruhland C, Robertson NP, Clayton A. Cerebrospinal fluid extracellular vesicle enrichment for protein biomarker discovery in neurological disease; multiple sclerosis. J Extracell Vesicles. 2017 Sep 3;6(1):1369805. 
12. Freeman SM, Samineni S, Allen PC, Stockinger D, Bales KL, Hwa GGC, et al. Plasma and CSF oxytocin levels after intranasal and intravenous oxytocin in awake macaques. Psychoneuroendocrinology. 2016 Apr;66:185-94.

13. Aleks, Ra D, David E, Castellano J. Aging. Aging-induced type I interferon signaling at the choroid plexus negatively affects brain function.

14. Worthington WC, Cathcart RS. Ependymal cilia: distribution and activity in the adult human brain. Science. 1963 Jan 18;139(3551):221-2.

15. Olstad EW, Ringers C, Hansen JN, Wens A, Brandt C, Wachten D, et al. Ciliary beating compartmentalizes cerebrospinal fluid flow in the brain and regulates ventricular development. Curr Biol. 2019 Jan 21;29(2):229-241.e6.

16. Lechtreck K-F, Delmotte P, Robinson ML, Sanderson MJ, Witman GB. Mutations in Hydin impair ciliary motility in mice. J Cell Biol. 2008 Feb 11;180(3):633-43.

17. Klarica M, Orešković D. Enigma of cerebrospinal fluid dynamics. Croat Med J. 2014 Aug;55(4):287-98.

18. Faubel R, Westendorf C, Bodenschatz E, Eichele G. Cilia-based flow network in the brain ventricles. Science. 2016 Jul 8;353(6295):176-8.

19. Boutin C, Labedan P, Dimidschstein J, Richard F, Cremer H, André P, et al. A dual role for planar cell polarity genes in ciliated cells. Proc Natl Acad Sci USA. 2014 Jul 29;111(30):E3129-38.

20. Spassky N, Merkle FT, Flames N, Tramontin AD, García-Verdugo JM, Alvarez-Buylla A. Adult ependymal cells are postmitotic and are derived from radial glial cells during embryogenesis. J Neurosci. 2005 Jan 5;25(1):10-8.

21. Yoo S, Cha D, Kim DW, Hoang T, Blackshaw S. Tanycyte-independent control of hypothalamic leptin signaling. BioRxiv. 2019 Jan 23;

22. Faouzi M, Leshan R, Björnholm M, Hennessey T, Jones J, Münzberg H. Differential accessibility of circulating leptin to individual hypothalamic sites. Endocrinology. 2007 Nov;148(11):5414-23.

23. Brightman M. W. and Palay S. L. The fine structure of ependyma in the brain of the rat. The Journal of Cell Biology. 1963 Volume 19 
24. Del Bigio MR. The ependyma: a protective barrier between brain and cerebrospinal fluid. Glia. 1995 May;14(1):1-13.

25. Del Bigio MR. Ependymal cells: biology and pathology. Acta Neuropathol. 2010 Jan;119(1):55-73.

26. Lim DA, Tramontin AD, Trevejo JM, Herrera DG, García-Verdugo JM, Alvarez-Buylla A. Noggin antagonizes BMP signaling to create a niche for adult neurogenesis. Neuron. 2000 Dec;28(3):713-26.

27. Ohata S, Alvarez-Buylla A. Planar organization of multiciliated ependymal (E1) cells in the brain ventricular epithelium. Trends Neurosci. 2016 Jun 13;39(8):543-51.

28. Götz M, Stoykova A, Gruss P. Pax6 controls radial glia differentiation in the cerebral cortex. Neuron. 1998 Nov;21(5):1031-44.

29. Abdi K, Lai C-H, Paez-Gonzalez P, Lay M, Pyun J, Kuo CT. Uncovering inherent cellular plasticity of multiciliated ependyma leading to ventricular wall transformation and hydrocephalus. Nat Commun. 2018 Apr 25;9(1):1655.

30. Zhang RL, Zhang ZG, Wang Y, LeTourneau Y, Liu XS, Zhang X, et al. Stroke induces ependymal cell transformation into radial glia in the subventricular zone of the adult rodent brain. J Cereb Blood Flow Metab. 2007 Jun;27(6):1201-12.

31. Langlet F, Mullier A, Bouret SG, Prevot V, Dehouck B. Tanycyte-like cells form a blood-cerebrospinal fluid barrier in the circumventricular organs of the mouse brain. J Comp Neurol. 2013 Oct 15;521(15):3389-405.

32. Rodriguez_Tanycyte_Review.pdf.

33. Rizzoti K, Lovell-Badge R. Pivotal role of median eminence tanycytes for hypothalamic function and neurogenesis. Mol Cell Endocrinol. 2017 Apr 15;445:7-13.

34. Gross PM, Weindl A. Peering through the windows of the brain. J Cereb Blood Flow Metab. 1987 Dec;7(6):663-72.

35. Mirzadeh Z, Kusne Y, Duran-Moreno M, Cabrales E, Gil-Perotin S, Ortiz C, et al. Biand uniciliated ependymal cells define continuous floor-plate-derived tanycytic territories. Nat Commun. 2017 Jan 9;8:13759. 
36. Mullier A, Bouret SG, Prevot V, Dehouck B. Differential distribution of tight junction proteins suggests a role for tanycytes in blood-hypothalamus barrier regulation in the adult mouse brain. J Comp Neurol. 2010 Apr 1;518(7):943-62.

37. Langlet F, Levin BE, Luquet S, Mazzone M, Messina A, Dunn-Meynell AA, et al. Tanycytic VEGF-A boosts blood-hypothalamus barrier plasticity and access of metabolic signals to the arcuate nucleus in response to fasting. Cell Metab. 2013 Apr 2;17(4):607-17.

38. Yoo S, Blackshaw S. Regulation and function of neurogenesis in the adult mammalian hypothalamus. Prog Neurobiol. 2018 Apr 6;170:53-66.

39. Noctor SC, Martinez-Cerdeño V, Kriegstein AR. Neural stem and progenitor cells in cortical development. Novartis Found Symp. 2007;288:59-73; discussion 73.

40. Thomas Voigt. Development of glial cells in the cerebral wall of ferrets: Direct tracing of their transformation from radial glia into astrocytes. The Journal of Comparative Neurology. $1989 ; 289: 74-88$

41. Lledo P-M, Saghatelyan A. Integrating new neurons into the adult olfactory bulb: joining the network, life-death decisions, and the effects of sensory experience. Trends Neurosci. 2005 May;28(5):248-54.

42. Luskin MB. Restricted proliferation and migration of postnatally generated neurons derived from the forebrain subventricular zone. Neuron. $1993 \mathrm{Jul}$;11(1):173-89.

43. Lois C, García-Verdugo JM, Alvarez-Buylla A. Chain migration of neuronal precursors. Science. 1996 Feb 16;271(5251):978-81.

44. Lois C, Alvarez-Buylla A. Long-distance neuronal migration in the adult mammalian brain. Science. 1994 May 20;264(5162):1145-8.

45. Tramontin AD, García-Verdugo JM, Lim DA, Alvarez-Buylla A. Postnatal development of radial glia and the ventricular zone (VZ): a continuum of the neural stem cell compartment. Cereb Cortex. 2003 Jun;13(6):580-7.

46. Obernier K, Alvarez-Buylla A. Neural stem cells: origin, heterogeneity and regulation in the adult mammalian brain. Development. 2019 Feb 18;146(4).

47. Doetsch F, Caillé I, Lim DA, García-Verdugo JM, Alvarez-Buylla A. Subventricular zone astrocytes are neural stem cells in the adult mammalian brain. Cell. 1999 Jun 11;97(6):703-16. 
48. Lacar B, Young SZ, Platel J-C, Bordey A. Gap junction-mediated calcium waves define communication networks among murine postnatal neural progenitor cells. Eur J Neurosci. 2011 Dec;34(12):1895-905.

49. Shen Q, Wang Y, Kokovay E, Lin G, Chuang S-M, Goderie SK, et al. Adult SVZ stem cells lie in a vascular niche: a quantitative analysis of niche cell-cell interactions. Cell Stem Cell. 2008 Sep 11;3(3):289-300.

50. Obernier K, Cebrian-Silla A, Thomson M, Parraguez JI, Anderson R, Guinto C, et al. Adult Neurogenesis Is Sustained by Symmetric Self-Renewal and Differentiation. Cell Stem Cell. 2018 Feb 1;22(2):221-234.e8.

51. Menn B, Garcia-Verdugo JM, Yaschine C, Gonzalez-Perez O, Rowitch D, AlvarezBuylla A. Origin of oligodendrocytes in the subventricular zone of the adult brain. J Neurosci. 2006 Jul 26;26(30):7907-18.

52. Mitchell DR. The evolution of eukaryotic cilia and flagella as motile and sensory organelles. Adv Exp Med Biol. 2007;607:130-40.

53. Satir P, Christensen ST. Structure and function of mammalian cilia. Histochem Cell Biol. 2008 Jun;129(6):687-93.

54. Barnes B. G. Ciliated secretory sells in the pars distalis of the mouse hypophysis. J. Ultrastructure Research 5. 1961; 453-467

55. Gray J. The mechanism of ciliary movement. JSTOR. 1921

56. Mitchell D. R. and Winfield S. S. Characterization of a Chlamydomonas Insertional Mutant that Disrupts Flagellar Central Pair Microtubule-associated Structures. The Journal of Cell Biology, Volume 144, Number 2, Jan. 25 1999; 293-304

57. Kamiya R. Exploring the function of inner and outer dynein arms with Chlamydomonas mutants. Cell Motil Cytoskeleton. 1995;32(2):98-102.

58. Teves ME, Nagarkatti-Gude DR, Zhang Z, Strauss JF. Mammalian axoneme central pair complex proteins: Broader roles revealed by gene knockout phenotypes. Cytoskeleton (Hoboken). 2016 Jan;73(1):3-22.

59. Sorokin SP. Reconstructions of centriole formation and ciliogenesis in mammalian lungs. J Cell Sci. 1968 Jun;3(2):207-30. 
60. Hua K, Ferland RJ. Primary Cilia Reconsidered in the Context of Ciliopathies: Extraciliary and Ciliary Functions of Cilia Proteins Converge on a Polarity theme? Bioessays. 2018 Aug;40(8):e1700132.

61. Satir P, Pedersen LB, Christensen ST. The primary cilium at a glance. J Cell Sci. 2010 Feb 15;123(Pt 4):499-503.

62. Uzbekov R, Alieva I. Who are you, subdistal appendages of centriole? Open Biol. 2018;8(7).

63. Mönnich M, Borgeskov L, Breslin L, Jakobsen L, Rogowski M, Doganli C, et al. CEP128 Localizes to the Subdistal Appendages of the Mother Centriole and Regulates TGFß/BMP Signaling at the Primary Cilium. Cell Rep. 2018 Mar 6;22(10):2584-92.

64. Chen JV, Kao L-R, Jana SC, Sivan-Loukianova E, Mendonça S, Cabrera OA, et al. Rootletin organizes the ciliary rootlet to achieve neuron sensory function in Drosophila. J Cell Biol. 2015 Oct 26;211(2):435-53.

65. Ishikawa H, Marshall WF. Mechanobiology of Ciliogenesis. Bioscience. 2014 Dec 1;64(12):1084-91.

66. Mochizuki T, Wu G, Hayashi T, Xenophontos SL, Veldhuisen B, Saris JJ, et al. PKD2, a gene for polycystic kidney disease that encodes an integral membrane protein. Science. 1996 May 31;272(5266):1339-42.

67. Yoder BK, Hou X, Guay-Woodford LM. The polycystic kidney disease proteins, polycystin-1, polycystin-2, polaris, and cystin, are co-localized in renal cilia. J Am Soc Nephrol. 2002 Oct;13(10):2508-16.

68. Retailleau K, Duprat F. Polycystins and partners: proposed role in mechanosensitivity. J Physiol (Lond). 2014 Jun 15;592(12):2453-71.

69. The European Polycystic Kidney Disease Consortium The Polycystic Kidney Disease 1 Gene Encodes a 14 kb Transcript and Lies within a Duplicated Region on Chromosome 16. Cell, Vol. 77, June 17 1994:881-894

70. Audrézet M-P, Cornec-Le Gall E, Chen J-M, Redon S, Quéré I, Creff J, et al. Autosomal dominant polycystic kidney disease: comprehensive mutation analysis of PKD1 and PKD2 in 700 unrelated patients. Hum Mutat. 2012 Aug;33(8):1239-50. 
71. Ohata S, Herranz-Pérez V, Nakatani J, Boletta A, García-Verdugo JM, Álvarez-Buylla A. Mechanosensory genes pkd1 and pkd2 contribute to the planar polarization of brain ventricular epithelium. J Neurosci. 2015 Aug 5;35(31):11153-68.

72. Gueron S, Levit-Gurevich K. Energetic considerations of ciliary beating and the advantage of metachronal coordination. Proc Natl Acad Sci USA. 1999 Oct 26;96(22):122405.

73. Dirksen E. Centriole morphogenesis in developing ciliated epithelium of the mouse oviduct. The Journal of Cell Biology Volume 51. 1971:268-302

74. Fritz-Laylin LK, Fulton C. Naegleria: a classic model for de novo basal body assembly. Cilia. 2016 Apr 4;5:10.

75. Kim H-K, Kang J-G, Yumura S, Walsh CJ, Cho JW, Lee J. De novo formation of basal bodies in Naegleria gruberi: regulation by phosphorylation. J Cell Biol. 2005 Jun 6;169(5):71924.

76. Werner ME, Hwang P, Huisman F, Taborek P, Yu CC, Mitchell BJ. Actin and microtubules drive differential aspects of planar cell polarity in multiciliated cells. J Cell Biol. 2011 Oct 3;195(1):19-26.

77. Matsuo M, Shimada A, Koshida S, Saga Y, Takeda H. The establishment of rotational polarity in the airway and ependymal cilia: analysis with a novel cilium motility mutant mouse. Am J Physiol Lung Cell Mol Physiol. 2013 Jun 1;304(11):L736-45.

78. Clare DK, Magescas J, Piolot T, Dumoux M, Vesque C, Pichard E, et al. Basal foot MTOC organizes pillar MTs required for coordination of beating cilia. Nat Commun. 2014 Sep $12 ; 5: 4888$.

79. Brooks ER, Wallingford JB. Multiciliated cells. Curr Biol. 2014 Oct 6;24(19):R97382.

80. Werner ME, Mitchell BJ. Planar cell polarity: microtubules make the connection with cilia. Curr Biol. 2012 Dec 4;22(23):R1001-4.

81. Wanner A, Salathé M, O'Riordan TG. Mucociliary clearance in the airways. Am J Respir Crit Care Med. 1996 Dec;154(6 Pt 1):1868-902.

82. Lyons RA, Saridogan E, Djahanbakhch O. The reproductive significance of human Fallopian tube cilia. Hum Reprod Update. 2006 Aug;12(4):363-72. 
83. Sawamoto K, Wichterle H, Gonzalez-Perez O, Cholfin JA, Yamada M, Spassky N, et al. New neurons follow the flow of cerebrospinal fluid in the adult brain. Science. 2006 Feb 3;311(5761):629-32.

84. Knowles MR, Boucher RC. Mucus clearance as a primary innate defense mechanism for mammalian airways. J Clin Invest. 2002 Mar;109(5):571-7.

85. Onoufriadis A, Paff T, Antony D, Shoemark A, Micha D, Kuyt B, et al. Splice-site mutations in the axonemal outer dynein arm docking complex gene CCDC114 cause primary ciliary dyskinesia. Am J Hum Genet. 2013 Jan 10;92(1):88-98.

86. Davis SD, Ferkol TW, Rosenfeld M, Lee H-S, Dell SD, Sagel SD, et al. Clinical features of childhood primary ciliary dyskinesia by genotype and ultrastructural phenotype. Am J Respir Crit Care Med. 2015 Feb 1;191(3):316-24.

87. Şişmanlar Eyüboğlu T, Aslan AT, Ceylan A, Soysal AŞ, Budakoğlu Iİ, Ulukavak Çiftçi $\mathrm{T}$, et al. Neurocognitive disorders and sleep in children with primary ciliary dyskinesia. Pediatr Pulmonol. 2018 Oct;53(10):1436-41.

88. Zariwala MA, Knowles MR, Omran H. Genetic defects in ciliary structure and function. Annu Rev Physiol. 2007;69:423-50.

89. Banizs B, Pike MM, Millican CL, Ferguson WB, Komlosi P, Sheetz J, et al. Dysfunctional cilia lead to altered ependyma and choroid plexus function, and result in the formation of hydrocephalus. Development. 2005 Dec;132(23):5329-39.

90. Ibañez-Tallon I, Pagenstecher A, Fliegauf M, Olbrich H, Kispert A, Ketelsen U-P, et al. Dysfunction of axonemal dynein heavy chain Mdnah5 inhibits ependymal flow and reveals a novel mechanism for hydrocephalus formation. Hum Mol Genet. 2004 Sep 15;13(18):213341.

91. Kelly M, Chen P. Shaping the mammalian auditory sensory organ by the planar cell polarity pathway. Int J Dev Biol. 2007;51(6-7):535-47.

92. Keller R. Shaping the vertebrate body plan by polarized embryonic cell movements. Science. 2002 Dec 6;298(5600):1950-4.

93. Wada $\mathrm{H}$, Okamoto H. Roles of noncanonical Wnt/PCP pathway genes in neuronal migration and neurulation in zebrafish. Zebrafish. 2009 Mar;6(1):3-8. 
94. Zallen JA. Planar polarity and tissue morphogenesis. Cell. 2007 Jun 15;129(6):105163.

95. Lawrence PA, Struhl G, Morata G. Bristle patterns and compartment boundaries in the tarsi of Drosophila. J Embryol Exp Morphol. 1979 Jun;51:195-208.

96. Axelrod JD. Unipolar membrane association of Dishevelled mediates Frizzled planar cell polarity signaling. Genes Dev. 2001 May 15;15(10):1182-7.

97. Strutt DI. Asymmetric localization of frizzled and the establishment of cell polarity in the Drosophila wing. Mol Cell. 2001 Feb;7(2):367-75.

98. Peng Y, Axelrod JD. Asymmetric protein localization in planar cell polarity: mechanisms, puzzles, and challenges. Curr Top Dev Biol. 2012;101:33-53.

99. Goodrich LV, Strutt D. Principles of planar polarity in animal development. Development. 2011 May;138(10):1877-92.

100. Adler P. N. Planar Signaling and Morphogenesis in Drosophila. Developmental Cell, Vol. 2, May 2002:525-535

101. Lawrence PA, Struhl G, Casal J. Planar cell polarity: one or two pathways? Nat Rev Genet. 2007 Jul;8(7):555-63.

102. Lawrence PA, Casal J. Planar cell polarity: two genetic systems use one mechanism to read gradients. Development. 2018 Dec 10;145(23).

103. Brittle AL, Repiso A, Casal J, Lawrence PA, Strutt D. Four-jointed modulates growth and planar polarity by reducing the affinity of dachsous for fat. Curr Biol. 2010 May 11;20(9):803-10.

104. Casal J, Struhl G, Lawrence PA. Developmental compartments and planar polarity in Drosophila. Curr Biol. 2002 Jul 23;12(14):1189-98.

105. Ma D, Yang C, McNeill H, Simon MA, Axelrod JD. Fidelity in planar cell polarity signalling. Nature. 2003 Jan 30;421(6922):543-7.

106. Casal J, Lawrence PA, Struhl G. Two separate molecular systems, Dachsous/Fat and Starry night/Frizzled, act independently to confer planar cell polarity. Development. 2006 Nov;133(22):4561-72. 
107. Strutt H, Gamage J, Strutt D. Robust Asymmetric Localization of Planar Polarity Proteins Is Associated with Organization into Signalosome-like Domains of Variable Stoichiometry. Cell Rep. 2016 Dec 6;17(10):2660-71.

108. Ohata S, Nakatani J, Herranz-Pérez V, Cheng J, Belinson H, Inubushi T, et al. Loss of Dishevelleds disrupts planar polarity in ependymal motile cilia and results in hydrocephalus. Neuron. 2014 Aug 6;83(3):558-71.

109. Vladar EK, Bayly RD, Sangoram AM, Scott MP, Axelrod JD. Microtubules enable the planar cell polarity of airway cilia. Curr Biol. 2012 Dec 4;22(23):2203-12.

110. Vladar EK, Lee YL, Stearns T, Axelrod JD. Observing planar cell polarity in multiciliated mouse airway epithelial cells. Methods Cell Biol. 2015 Mar 7;127:37-54.

111. By G, GARClA-BELLIDO. A genetic analysis of the determination of.

112. Wallingford JB. Planar cell polarity signaling, cilia and polarized ciliary beating. Curr Opin Cell Biol. 2010 Oct;22(5):597-604.

113. Mitchell B, Stubbs JL, Huisman F, Taborek P, Yu C, Kintner C. The PCP pathway instructs the planar orientation of ciliated cells in the Xenopus larval skin. Curr Biol. 2009 Jun 9;19(11):924-9.

114. De Iongh R, Rutland J. Orientation of respiratory tract cilia in patients with primary ciliary dyskinesia, bronchiectasis, and in normal subjects. J Clin Pathol. 1989 Jun;42(6):6139.

115. Hagiwara H, Kano A, Aoki T, Ohwada N, Takata K. Localization of gamma-tubulin to the basal foot associated with the basal body extending a cilium. Histochem J. 2000 Nov;32(11):669-71.

116. Kunimoto K, Yamazaki Y, Nishida T, Shinohara K, Ishikawa H, Hasegawa T, et al. Coordinated ciliary beating requires Odf2-mediated polarization of basal bodies via basal feet. Cell. 2012 Jan 20;148(1-2):189-200.

117. Tateishi K, Yamazaki Y, Nishida T, Watanabe S, Kunimoto K, Ishikawa H, et al. Two appendages homologous between basal bodies and centrioles are formed using distinct Odf2 domains. J Cell Biol. 2013 Nov 11;203(3):417-25.

118. Mitchell B, Jacobs R, Li J, Chien S, Kintner C. A positive feedback mechanism governs the polarity and motion of motile cilia. Nature. 2007 May 3;447(7140):97-101. 
119. Vladar EK, Axelrod JD. Dishevelled links basal body docking and orientation in ciliated epithelial cells. Trends Cell Biol. 2008 Nov;18(11):517-20.

120. Mirzadeh Z, Han Y-G, Soriano-Navarro M, García-Verdugo JM, Alvarez-Buylla A. Cilia organize ependymal planar polarity. J Neurosci. 2010 Feb 17;30(7):2600-10.

121. Kyrousi C, Lygerou Z, Taraviras S. How a radial glial cell decides to become a multiciliated ependymal cell. Glia. 2017 Feb 7;65(7):1032-42.

122. Al Jord A, Shihavuddin A, Servignat d'Aout R, Faucourt M, Genovesio A, Karaiskou A, et al. Calibrated mitotic oscillator drives motile ciliogenesis. Science. 2017 Nov 10;358(6364):803-6.

123. Al Jord A, Spassky N, Meunier A. Motile ciliogenesis and the mitotic prism. Biol Cell. 2019 Mar 24;

124. Hirota Y, Meunier A, Huang S, Shimozawa T, Yamada O, Kida YS, et al. Planar polarity of multiciliated ependymal cells involves the anterior migration of basal bodies regulated by non-muscle myosin II. Development. 2010 Sep;137(18):3037-46.

125. Gomperts BN, Gong-Cooper X, Hackett BP. Foxj1 regulates basal body anchoring to the cytoskeleton of ciliated pulmonary epithelial cells. J Cell Sci. 2004 Mar 15;117(Pt 8):1329_ 37.

126. Park TJ, Mitchell BJ, Abitua PB, Kintner C, Wallingford JB. Dishevelled controls apical docking and planar polarization of basal bodies in ciliated epithelial cells. Nat Genet. 2008 Jul;40(7):871-9.

127. Park TJ, Haigo SL, Wallingford JB. Ciliogenesis defects in embryos lacking inturned or fuzzy function are associated with failure of planar cell polarity and Hedgehog signaling. Nat Genet. 2006 Mar;38(3):303-11.

128. Blatt EN, Yan XH, Wuerffel MK, Hamilos DL, Brody SL. Forkhead transcription factor HFH-4 expression is temporally related to ciliogenesis. Am J Respir Cell Mol Biol. 1999 Aug;21(2):168-76.

129. Pelletier GJ, Brody SL, Liapis H, White RA, Hackett BP. A human forkhead/wingedhelix transcription factor expressed in developing pulmonary and renal epithelium. Am $\mathbf{J}$ Physiol. 1998;274(3):L351-9. 
130. Jacquet BV, Salinas-Mondragon R, Liang H, Therit B, Buie JD, Dykstra M, et al. FoxJ1dependent gene expression is required for differentiation of radial glia into ependymal cells and a subset of astrocytes in the postnatal brain. Development. 2009 Dec;136(23):4021-31.

131. Abedalthagafi MS, Wu MP, Merrill PH, Du Z, Woo T, Sheu S-H, et al. Decreased FOXJ1 expression and its ciliogenesis programme in aggressive ependymoma and choroid plexus tumours. J Pathol. 2016 Mar;238(4):584-97.

132. Avasthi P, Marshall WF. Stages of ciliogenesis and regulation of ciliary length. Differentiation. 2012 Feb;83(2):S30-42.

133. Takagishi M, Sawada M, Ohata S, Asai N, Enomoto A, Takahashi K, et al. Daple coordinates planar polarized microtubule dynamics in ependymal cells and contributes to hydrocephalus. Cell Rep. 2017 Jul 25;20(4):960-72.

134. Guirao B, Meunier A, Mortaud S, Aguilar A, Corsi J-M, Strehl L, et al. Coupling between hydrodynamic forces and planar cell polarity orients mammalian motile cilia. Nat Cell Biol. 2010 Apr;12(4):341-50.

135. Tissir F, Qu Y, Montcouquiol M, Zhou L, Komatsu K, Shi D, et al. Lack of cadherins Celsr2 and Celsr3 impairs ependymal ciliogenesis, leading to fatal hydrocephalus. Nat Neurosci. 2010 Jun;13(6):700-7.

136. Schwartz W. J., Gainer H. Suprachiasmatic Nucleus: Use of C-Labeled Deoxyglucose Uptake as a Functional Marker. Jan 1977

137. Buijs RM, Kalsbeek A. Hypothalamic integration of central and peripheral clocks. Nat Rev Neurosci. 2001 Jul;2(7):521-6.

138. Matsuo T, Yamaguchi S, Mitsui S, Emi A, Shimoda F, Okamura H. Control mechanism of the circadian clock for timing of cell division in vivo. Science. 2003 Oct 10;302(5643):2559.

139. Brown SA, Ripperger J, Kadener S, Fleury-Olela F, Vilbois F, Rosbash M, et al. PERIOD1-associated proteins modulate the negative limb of the mammalian circadian oscillator. Science. 2005 Apr 29;308(5722):693-6.

140. Kowalska E, Ripperger JA, Hoegger DC, Bruegger P, Buch T, Birchler T, et al. NONO couples the circadian clock to the cell cycle. Proc Natl Acad Sci USA. 2013 Jan 29;110(5):1592-9. 
141. Ohtani N, Yamokoshi K, Takahashi A, Hara E. The p16INK4a-RB pathway: molecular link between cellular senescence and tumor suppression. The journal of medical investigation. Vol.51 2004

142. Tennessen JM, Gardner HF, Volk ML, Rougvie AE. Novel heterochronic functions of the Caenorhabditis elegans period-related protein LIN-42. Dev Biol. 2006 Jan 1;289(1):30-43.

143. McCulloch KA, Rougvie AE. Caenorhabditis elegans period homolog lin-42 regulates the timing of heterochronic miRNA expression. Proc Natl Acad Sci USA. 2014 Oct 28;111(43):15450-5.

144. Perales R, King DM, Aguirre-Chen C, Hammell CM. LIN-42, the Caenorhabditis elegans PERIOD homolog, negatively regulates microRNA transcription. PLoS Genet. 2014 Jul 17;10(7):e1004486.

145. Jeon M, Gardner HF, Miller EA, Deshler J, Rougvie AE. Similarity of the C. elegans developmental timing protein LIN-42 to circadian rhythm proteins. Science. 1999 Nov $5 ; 286(5442): 1141-6$.

146. Abrahante JE, Miller EA, Rougvie AE. Identification of heterochronic mutants in Caenorhabditis elegans. Temporal misexpression of a collagen::green fluorescent protein fusion gene. Genetics. 1998 Jul;149(3):1335-51.

147. Frangi AF, Niessen WJ, Vincken KL, Viergever MA. Multiscale vessel enhancement filtering. In: Wells WM, Colchester A, Delp S, editors. Medical Image Computing and Computer-Assisted Intervention - MICCAI'98. Berlin, Heidelberg: Springer Berlin Heidelberg; 1998. p. 130-7.

148. Ferenczy A, Richart R. M, Agate F. J, Purkerson M. L, Dempsey E. W. Scanning Electron Microscopy of the Human Fallopian Tube. Science 1097

149. Hashemi MM, Holden BS, Taylor MF, Wilson J, Coburn J, Hilton B, et al. Antibacterial and Antifungal Activities of Poloxamer Micelles Containing Ceragenin CSA-131 on Ciliated Tissues. Molecules. 2018 Mar 7;23(3).

150. Balland E, Dam J, Langlet F, Caron E, Steculorum S, Messina A, et al. Hypothalamic tanycytes are an ERK-gated conduit for leptin into the brain. Cell Metab. 2014 Feb 4;19(2):293301. 
151. Yin H, Copley CO, Goodrich LV, Deans MR. Comparison of phenotypes between different vangl2 mutants demonstrates dominant effects of the Looptail mutation during hair cell development. PLoS One. 2012 Feb 20;7(2):e31988.

152. Chien Y-H, Werner ME, Stubbs J, Joens MS, Li J, Chien S, et al. Bbof1 is required to maintain cilia orientation. Development. 2013 Aug;140(16):3468-77.

153. Luo J, Shook BA, Daniels SB, Conover JC. Subventricular zone-mediated ependyma repair in the adult mammalian brain. J Neurosci. 2008 Apr 2;28(14):3804-13.

154. Luo Y, Coskun V, Liang A, Yu J, Cheng L, Ge W, et al. Single-cell transcriptome analyses reveal signals to activate dormant neural stem cells. Cell. 2015 May 21;161(5):117586.

155. Monsalve GC, Van Buskirk C, Frand AR. LIN-42/PERIOD controls cyclical and developmental progression of C. elegans molts. Curr Biol. 2011 Dec 20;21(24):2033-45.

156. Liu T, Jin X, Prasad RM, Sari Y, Nauli SM. Three types of ependymal cells with intracellular calcium oscillation are characterized by distinct cilia beating properties. J Neurosci Res. 2014 Sep;92(9):1199-204.

157. Tsuji T, Nakamura R, Katsuno T, Kishimoto Y, Suehiro A, Yamashita M, et al. Longterm preservation of planar cell polarity in reversed tracheal epithelium. Respir Res. 2018 Feb 2;19(1):22.

158. Iliff JJ, Wang M, Zeppenfeld DM, Venkataraman A, Plog BA, Liao Y, et al. Cerebral arterial pulsation drives paravascular CSF-interstitial fluid exchange in the murine brain. $\mathbf{J}$ Neurosci. 2013 Nov 13;33(46):18190-9.

159. Bolborea M, Dale N. Hypothalamic tanycytes: potential roles in the control of feeding and energy balance. Trends Neurosci. 2013 Feb;36(2):91-100.

160. Laplante M, Sabatini DM. mTOR signaling in growth control and disease. Cell. 2012 Apr 13;149(2):274-93.

161. Saucedo LJ, Edgar BA. Why size matters: altering cell size. Curr Opin Genet Dev. 2002 Oct;12(5):565-71.

162. Lloyd AC. The regulation of cell size. Cell. 2013 Sep 12;154(6):1194-205. 
163. Husse JL. Genetic disruption of the master pacemaker in the suprachiasmatic nucleus sheds light on the hierarchical organization of the mammalian circadian timing system.

164. Zheng B, Albrecht U, Kaasik K, Sage M, Lu W, Vaishnav S, et al. Nonredundant roles of the mPer1 and mPer2 genes in the mammalian circadian clock. Cell. 2001 Jun 1;105(5):68394.

165. Lee C, Etchegaray JP, Cagampang FR, Loudon AS, Reppert SM. Posttranslational mechanisms regulate the mammalian circadian clock. Cell. 2001 Dec 28;107(7):855-67.

166. Curtin JA, Quint E, Tsipouri V, Arkell RM, Cattanach B, Copp AJ, et al. Mutation of Celsr1 disrupts planar polarity of inner ear hair cells and causes severe neural tube defects in the mouse. Curr Biol. 2003 Jul 1;13(13):1129-33.

167. Montcouquiol M, Rachel RA, Lanford PJ, Copeland NG. Identification of Vangl2 and Scrb1 as planar polarity genes in mammals. nature.com.

168. Lu X, Borchers AGM, Jolicoeur C, Rayburn H, Baker JC. PTK7/CCK-4 is a novel regulator of planar cell polarity in vertebrates. nature.com.

169. Montcouquiol M, Sans N, Huss D, Kach J. Asymmetric localization of Vangl2 and Fz3 indicate novel mechanisms for planar cell polarity in mammals. Soc Neuroscience. 


\section{Acknowledgments}

At first, I would like to express my sincere gratitude for Prof. Gregor Eichele, who is an excellent teacher and inspired me with his great sense of organization and incredible knowledge. He paid interest in my ideas and provoked stimulating and meaningful discussions that guided me throughout the past four years. I am thankful for his advice and guidance, because of which I learned great lessons in science and in life.

Despite my supervisor, I am also deeply thankful to Prof. Bodenschatz and Prof. Hoyer Fender. During TAC meetings or other encounters, their challenging questions and useful methodological suggestions were a great help to understand my project to a greater depth. Especially, the collaboration with the group of Prof. Bodenschatz enabled us to bring the expertise of the field of Biology and Physics together. This combined work is one of the strengths of this study.

A very special thanks goes to Dr. Christian Westendorf, whose constant help and incredible skills as a biophysicist upgraded the quality of my research. Despite, the friendship that developed in the past years has helped me go through difficult times.

I also thank my fellow lab mates and friends at the MPI BPC for the greatest lunchtimes and coffee breaks while having the most interesting discussions. In particular, the ones with Prof. Anastasia Stoykova have helped me understand my project from a different perspective and raise relevant scientific questions.

Now, I would like to thank my loved-ones. My parents (Madan \& Anita Kapoor), my aunt (Mamta Kapoor), my siblings (Kevin Kapoor \& Nandika Wadhwa-Kapoor), and last but not least my boyfriend (Tom Brown). They have tolerated my madness through a variety of times and always gave me strength and security. I felt free in my mind because their support was always with me

Thank you

Shoba Kapoor 


\section{CV}

Shoba Kapoor

\section{Personal Data}

Date of birth:

Place of birth:

Nationality:

Gender:

\section{Education}

$11 / 2015$ - present

$10 / 2012-09 / 2014$

$10 / 2009-09 / 2012$

$08 / 2003-05 / 2008$
$10 / 11 / 1989$

Afghanistan - Kabul

German

Female
Max Planck Institute for Biophysical Chemistry

Ph.D. in the program Genes and Development of the graduate school Göttingen Graduate Center for Neurosciences, Biophysics, and Molecular Biosciences (GGNB) of the Georg August University Göttingen

\section{Georg August University Göttingen}

Master's in Developmental, Neural and Behavioral Biology

Georg August University Göttingen

Bachelor's in Biology

Gymnasium Rodenkirchen, Cologne

Abitur

\section{Laboratory experience}

$11 / 2015$ - present
Ph.D. under the supervision of Prof. Dr. Gregor Eichele

Title: Spatio-temporal dynamics in the anchoring of ependymal cilia.

Department of Genes and Behavior

Max Planck Institute for Biophysical Chemistry, Göttingen 
Title: "Sialic acids and their binding properties"

Department of Reconstructive Neurobiology

Life and Brain Center, University of Bonn

$05 / 2013-08 / 2013$

Internship under the supervision of Dr. Igor Kagan

Decision and Awareness Group

German Primate Center, Göttingen

$04 / 2012-09 / 2012$

Bachelor thesis under the supervision of Prof. Dr. Gregor Eichele

Title: "Histochemical confirmation of choroid plexus specific

deletion of the clock gene Bmal1"

Department of Genes and Behavior

Max Planck Institute of Biophysical Chemistry, Göttingen

\section{Stay abroad}
$08 / 2008-02 / 2009$
Work and Travel in Australia
$10 / 2014-02 / 2015$
Traveling in South America (Peru and Bolivia)

\section{Work experience}

$02 / 2014-09 / 2014$

Student assistant of Prof. Dr. Harald Neumann

Reconstructive Neurobiology

Life and Brain Center, University of Bonn

Responsible for animal house facility

Preparation of buffers, cell culture media, etc.

01/2013 - 03/2013 Student assistant of Prof. Dr. Treue

German Primate Center, Göttingen

Evaluation of answer sheets of the exam: Biopsychology written by psychology and biology bachelor students 


\section{Publications}

Shahraz, A., Kopatz, J., Mathy, R., Kappler, J., Winter, D., Kapoor, S., ... Neumann, H. (2015). Antiinflammatory activity of low molecular weight polysialic acid on human macrophages. Scientific reports, 5, 16800. doi:10.1038/srep16800

\section{Poster}

1. Module Neurobiology 2 at Georg August University Göttingen

Title: "Changing saccade plans: response competition in spatial decision - making"

2. EMBO Cilia Meeting, Copenhagen

Title: "Spatio-temporal dynamics in the anchoring of cilia"

3. Unity and Diversity of ciliary systems in locomotion and transport, London

Title: "Spatio-temporal dynamics in the anchoring of cilia"

Shoba Kapoor

Göttingen, 31.07.2019 